\title{
The Operator Quantization of the Open Bosonic String: Field Algebra
}

\author{
Hendrik Grundling ${ }^{1}$ and C.A. Hurst ${ }^{2}$ \\ ${ }^{1}$ Department of Mathematics, University of New South Wales, P.O. Box 1, Kensington, \\ New South Wales, Australia 2033 \\ ${ }^{2}$ Department of Physics and Mathematical Physics, University of Adelaide, GPO Box 498, \\ Adelaide, S.A. 5001, Australia
}

Received July 25, 1991; in revised form January 5, 1993

\begin{abstract}
Our aim in this paper is to make explicit the operator theory of the heuristic open Bosonic string and to abstract a suitable field algebra for the string. This is done on a Fock-Krein space and we examine integrability and J-unitary implementability of all the defining transformations of the string, i.e. time translations, gauge transformations and Poincaré transformations. The results obtained agree partially with those of Bowick and Rajeev, i.e. the gauge transformations do not leave the Fock-Krein complex structure invariant. Once we obtained integrated transformation groups on a suitable symplectic space for the infinitesimal transformations of the string, and proved implementability of these for the FockKrein representation, we are then free to define an abstract $C^{*}$-algebra carrying all the algebraic information of the string, and to examine different representations.
\end{abstract}

\section{Introduction}

There are several rigorous approaches to the open bosonic quantum string, of which we find the geometric approach of Bowick and Rajeev $[16,17]$ and Mickelsson [15] most appealing. This approach produced the following results:

(i) The complex structure $K$ which defines the quantum Hilbert space for the string, is not invariant under the gauge group $\operatorname{Diff}^{+} S^{1}$.

(ii) The orbit of $K$ under Diff ${ }^{+} S^{1}$ is taken as the dynamical manifold, endowed with the topology of the homogeneous space Diff ${ }^{+} S^{1} / S^{1}$ which is bijective to it. This manifold has a Kähler (hence symplectic) structure, on which one constructs a Fock bundle $\mathscr{B}$ using the complex structure at each point to obtain the one particle spaces. The curvature of this bundle produces the Virasoro anomaly as a two-cocycle. Mickelsson [15] showed that $\mathscr{B}$ has no $\operatorname{Diff}^{+} S^{1}$ invariant sections, but it is possible to adjoin a fermionic ghost bundle to $\mathscr{B}$ which produces a bundle with such sections in dimension 26.

However, geometric quantization is not yet a full quantization, so there is still some mathematical distance between the heuristic quantum string and the geometric 
approach. In regard to the heuristic quantum string, this has several unpleasant features: the representation space has indefinite metric, there is an anomalous central term appearing in the commutation relations of the (infinitesimal) gauge transformations, and when the constraint set is split in half via a Gupta-Bleuler technique to obtain a first-class set, there is no vector which can annihilate these constraints due to spectrum problems. However, the string is an infinite dimensional theory, so it has many inequivalent representations, and it makes sense to look for representations of the string which are better behaved. This is the rationale behind the present construction of an abstract field algebra for the string.

The first part in constructing a field algebra for the open covariant bosonic string (henceforth shortened to "the string") is to define the operator theory on the appropriate indefinite inner product space (abbreviated to IIP-space), remaining as close as possible to the heuristic operator formulation $[1,2]$ and ignoring the geometry of the string. Within this functional analytic framework we then wish to examine similar issues and their consequences to those examined in the geometric framework. We hope that this can fill the gap pointed out by Alvarez-Gaumé and Gomez [18] viz. the lack of a natural operator formulation for the string. We then generalize the operator theory to a suitable $\mathrm{C}^{*}$-algebra framework, that is, we construct a unital $\mathrm{C}^{*}$-algebra which reproduces all the algebraic structures which define the operators of the string (in integrated form).

The chosen heuristic formulation of the quantum string, being the historical precedent [1] of more recent formulations, has been referred to as "old quantization" [12] in contradistinction with path integral quantization. We prefer this "old" formulation since operator quantization can at least in principle be mathematically well defined in terms of operators on some space (Hilbert, rigged Hilbert or IIP-space), whereas the measure for the path integral of a quantum field does not exist in general (except in the Euclidean case), and for most field theories it has not been demonstrated that path integral quantization produces an equivalent or larger theory to that of operator quantization.

To the best of our knowledge whilst this article was written, there was only one attempt [13] to construct the string field algebra as a $C^{*}$-algebra, but this suffered from being mathematically undefined at a crucial step, and in being incomplete by not containing the excitation mode oscillators of the string. A possible constraint $\mathrm{C}^{*}$-algebra for the string was also considered in [14]. However, very recently Wiesbrock [34] obtained a $\mathrm{C}^{*}$-algebra for the string using a totally different approach to ours. This will be examined in the addendum.

In this paper we start at a fairly concrete level. First, since physics is defined at the infinitesimal level, and the $\mathrm{C}^{*}$-algebras deal with the integrated (exponentiated) operators, it is necessary to consider integrability questions for the time evolutions and gauge transformations. An exponentiation for an operator on IIP-space or on symplectic space need not be defined nor unique (the power series need not converge, and a spectral theory may not exist), we need to reconstruct the heuristic infinitesimal theory carefully as well-defined operators on the correct Fock-Krein space [5], and in this framework examine possible exponentiation methods. Quasiunitary implementability is also naturally exposed in this framework. The infinitesimal gauge transformations of the canonical variables (obtained from commutators), do not commute with the complex structure of the Krein oneparticle space, as a result of which exponentiation becomes a complicated (but tractable) affair. This noncommutativity partially agrees with Bowick and Rajeev's finding mentioned under item (i) above. 
The paper runs as follows. In Sect. 1 we sketch the bare bones of the heuristic quantum string for later reference, whilst in Sect. 2 the appropriate one particle Krein space is obtained in order to start a Fock construction in Sect. 3. There the mode oscillator operators and (Virasoro) string constraint operators are defined on the Fock-Krein space, domain conditions considered and the full (infinitesimal) operator string theory written down in the Fock-Krein representation. Section 4 consists of the integration of the infinitesimally symplectic time translations to a symplectic one parameter group on test function space, together with the demonstration that this one parameter group is J-unitary implementable on the string field operators in the Fock-Krein representation. However on attempting to do this for the infinitesimally symplectic gauge transformations we ran into problems. These transformations do not commute with the Krein complex structure and are not amenable to Krein spectral theory, so exponentiation is difficult. The exponential series for these transformations turns out to have unpleasant convergence behaviour, and when it does converge, it is to an unbounded operator. This forces us to make decompositions of the infinitesimal gauge transformations into components which are separately integrable but do not commute. These integrated components are implementable in the Fock-Krein representation, and we use the Trotter product formula on their implementers to finally obtain the full integrated gauge transformations on symplectic space together with the implementers of the latter. In Sect. 6 we cast all this in $\mathrm{C}^{*}$-algebra format, use CCR-algebras for the mode fields, show there is an auxiliary $\mathrm{C}^{*}$-algebra for each Fock-Krein representation on which the string structures can be examined to yield information for the usual theory, and we propose a $C^{*}$-algebra for the field algebra of the string, together with constraint conditions to select its physical states.

\section{Heuristic Structures of the Open Covariant Bosonic String}

For extensive reviews of the string, we refer to $[1,2,12]$. We present here the essential heuristic structures.

All operators act on an indefinite inner product (IIP) space which is a Fock construction on the one particle space $M^{D+1} \otimes l^{2}$, where $M^{D+1}$ is Minkowski space equipped with the usual pseudo-Riemannian metric $g$ with signature $(1, D)$. At this level domain problems etc. are not considered, and manipulations with operators are only formal. This will be firmed up in the following sections.

The centre of mass coordinates and total momentum of the string is described by a canonical pair $q^{\mu}, p^{\mu}$ satisfying the canonical commutation relations (CCR): $\left[p^{\mu}, q^{\nu}\right]=i g^{\mu \nu}$ with $g^{\mu \nu}=\operatorname{diag}(-1,+1, \ldots,+1), \mu, \nu=0,1,2, \ldots, D$ the Minkowski metric on $(D+1)$ space-time. The appearance of $g^{\mu \nu}$ in the CCR's indicate why the one-particle space is an IIP-space.

Together with the centre of mass motion, a string is described by a denumerable infinity of excitation modes, expressed as harmonic oscillators via creation and annihilation operators $a_{n}^{\mu}, a_{m}^{v *}$ satisfying

$$
\left[a_{n}^{\mu}, a_{m}^{\nu *}\right]=-g^{\mu v} \delta_{n, m}, \quad n, m \geqq 1
$$

and this infinity of oscillators indicate the field theoretical aspect of the string. Adjoints are in terms of the IIP. 
The constaints for the string, which generate the world-sheet parametrizations are:

$$
\begin{gathered}
L_{n}:=\frac{1}{4 \alpha^{\prime}} \sum_{m=-\infty}^{+\infty}: \alpha_{m}^{\mu} \alpha_{n-m \mu}:, \quad n \in \mathbb{Z}, \text { where: } \\
\alpha_{0}^{\mu}=2 \alpha^{\prime} p^{\mu}, \quad \alpha_{-n}^{\mu}=\left(\alpha_{n}^{\mu}\right)^{*}, \quad \alpha_{n}^{\mu}:=a_{n}^{\mu} \sqrt{2 \alpha^{\prime} n} \quad \forall n \geqq 1,
\end{gathered}
$$

and normal ordering is with relation to $a_{n}^{\mu}$ and $a_{n}^{\mu *}$. Note that $L_{0}=L_{0}^{*}$ and $L_{n}^{*}=L_{-n}$. In terms of $a_{n}^{\mu}$ we have

$$
\begin{aligned}
L_{0} & =\frac{1}{4 \alpha^{\prime}} \sum_{k=-\infty, \neq 0}^{+\infty} 2 \alpha^{\prime}|k|: a_{k} \cdot a_{-k}:+\frac{1}{4 \alpha^{\prime}}:\left(2 \alpha^{\prime} p^{\mu}\right)\left(2 \alpha^{\prime} p_{\mu}\right): \\
& =\sum_{k=1}^{\infty} k a_{k}^{*} \cdot a_{k}+\alpha^{\prime} p \cdot p
\end{aligned}
$$

and for $n>0$ :

$$
\begin{aligned}
L_{n}= & \frac{1}{4 \alpha^{\prime}} \sum_{\substack{k=-\infty \\
n \neq k \neq 0}}^{+\infty} \sqrt{2 \alpha^{\prime}|k|} \sqrt{2 \alpha^{\prime}|k-n|}: a_{k} \cdot a_{n-k}:+\frac{1}{4 \alpha^{\prime}}\left(2 \alpha^{\prime} p^{\mu}\right) a_{n \mu} \sqrt{2 \alpha^{\prime} n} \\
& +\frac{1}{4 \alpha^{\prime}} a_{n}^{\mu} \sqrt{2 \alpha^{\prime} n}\left(2 \alpha^{\prime} p_{\mu}\right) \\
= & \frac{1}{2} \sum_{-k=1}^{\infty} a_{|k|}^{*} \cdot a_{n+|k|} \sqrt{|k(k-n)|}+\frac{1}{2} \sum_{k=1}^{n-1} a_{k} \cdot a_{n-k} \sqrt{k(n-k)} \\
& +\frac{1}{2} \sum_{k=n+1}^{\infty} a_{k-n}^{*} \cdot a_{k} \sqrt{k(k-n)}+a_{n} \cdot p \sqrt{2 \alpha^{\prime} n} \\
= & \sum_{k=1}^{\infty} a_{k}^{*} \cdot a_{n+k} \sqrt{k(k+n)}+\frac{1}{2} \sum_{k=1}^{n-1} a_{k} \cdot a_{n-k} \sqrt{k(n-k)}+a_{n} \cdot p \sqrt{2 \alpha^{\prime} n},
\end{aligned}
$$

where we used the notation $b \cdot d:=b_{\mu} d^{\mu}$ and $a_{-n}:=a_{n}^{*}, n \geqq 1$. Note that normal ordering only affects the expression $L_{0}$, but not $L_{n}$ for $n \neq 0$. The Hamiltonian is usually taken as $H=L_{0}$. A trivial but tedious calculation employing only the CCR's gives:

$$
\left[L_{n}, L_{m}\right]=(n-m) L_{n+m}+\frac{D+1}{12} n\left(n^{2}-1\right) \delta_{n+m, 0} \quad \forall n, m \in \mathbb{Z},
$$

the Virasoro relations. At the classical level, the central term is absent in the corresponding Poisson bracket relation, and the Lie algebra thus produced, is the complexification of the Lie algebra of the group Diff ${ }^{+} S^{1}$, expressing the worldsheet reparametrizations of the string. The central term appears in the quantum theory as a result of the normal ordering in the definition of $L_{n}$, and if one took the whole gauge generator set $\left\{L_{n} \mid n \in \mathbb{Z}\right\}$ as the constraint set, the central term would make this set second-class whereas the classical set is first-class. This is therefore an anomaly, and one would expect the quantum gauge group to be some central extension of Diff ${ }^{+} S^{1}$ (cf. [3]). However this problem of the quantum theory is circumvented by the choice of constraint set $\left\{L_{n}-\alpha(0) \delta_{n, 0} \mid n \geqq 0\right\}$, which is 
first-class, and imposed through the selection criterion for physical states $|\psi\rangle$ :

$$
\left(L_{n}-\alpha(0) \delta_{n, 0}\right)|\psi\rangle=0 \quad \forall n \geqq 0,
$$

where $\alpha(0) \in \mathbb{R}$ is fixed by the physics. At this point all the boundary conditions of the string have been incorporated. Notice however that the constraints are nonhermitian for $n \neq 0$.

As for the physical transformations of the theory, the Hamiltonian $H=L_{0}$ gives the evolution of the system in terms of the internal proper time parameter $\tau$ on the world-sheet, hence does not destroy covariance in total space-time.

Poincare transformations of the string are induced by the generators $p_{\mu}$ and

$$
M_{\mu \nu}:=q^{\mu} p^{\nu}-q^{v} p^{\mu}-i \sum_{n=1}^{\infty}\left(\alpha_{-n}^{\mu} \alpha_{n}^{v}-\alpha_{-n}^{v} \alpha_{n}^{\mu}\right) / 2 n \alpha^{\prime},
$$

$u \neq v$, and these satisfy the Poincare algebra relations:

$$
\begin{gathered}
{\left[p_{\mu}, p_{v}\right]=0, \quad\left[p^{\mu}, M^{v \rho}\right]=i g^{\mu \rho} p^{v}-i g^{\mu \nu} p^{\rho},} \\
{\left[M^{\mu \nu}, M^{\rho \lambda}\right]=i g^{\mu \rho} M^{v \lambda}-i g^{v \rho} M^{\mu \lambda}+i g^{v \lambda} M^{\mu \rho}-i g^{\mu \lambda} M^{v \rho} .}
\end{gathered}
$$

Since $\left[L_{n}, p_{\mu}\right]=0=\left[L_{n}, M^{\mu \nu}\right]$, the Poincare transformations leave the constraints invariant, and only induce automorphisms on the set of canonical variables:

$$
\left\{q_{\mu}, p_{\mu}, a_{n}^{\mu}, a_{n}^{\mu *} \mid \mu=0, \ldots, D ; n \geqq 1\right\}
$$

\section{The One-Particle Space}

Guided by the field-theoretic aspect of the string, we aim to define the string operators as acting on a Fock space, and hence need to specify some one-particle space. This is also the appropriate level at which to inject the necessary geometry into the quantum theory, e.g. by choosing the one particle space as an $L^{2}$-space on a configuration manifold (possibly curved). In heuristic quantum theory, the one-particle space arises as the closure in some Hilbert norm of the set of smearing functions of the field operators. If the field operators are required to satisfy a field equation, the smearing functions are traditionally chosen as the solution space of the conjugate field equation. Typically, the space of smearing functions is a subspace of Schwartz space on the configuration manifold. In the following we will make the simplest possible choice of geometry, i.e. flat spaces throughout.

First, rewrite the heuristic theory in a convenient field operator format. The basic objects are the excitation mode oscillators $\left\{\alpha_{n} \mid n \in \mathbb{Z}\right\}$,

$$
\left[\alpha_{n}^{\mu}, \alpha_{m}^{v}\right]=-2 \alpha^{\prime} n g^{\mu v} \delta_{n+m, 0} \quad \forall n \in \mathbb{Z}
$$

and write these as canonical variables:

$$
q_{n}^{\mu}:=\frac{1}{\sqrt{2}}\left(\alpha_{-n}^{\mu}+\alpha_{n}^{\mu}\right), \quad p_{n}^{\mu}:=\frac{i}{\sqrt{2}}\left(\alpha_{-n}^{\mu}-\alpha_{n}^{\mu}\right)
$$

for all $n \geqq 1$, whence $\left[p_{n}^{\mu}, q_{m}^{v}\right]=2 i \alpha^{\prime} n \delta_{n, m} g^{\mu v}, n, m \geqq 1$. Add to this set the centre of mass variables

$$
q_{0}^{\mu}:=q^{\mu}, \quad p_{0}^{\mu}:=p^{\mu}, \quad\left[p_{0}^{\mu}, q_{0}^{\nu}\right]=i g^{\mu \nu}
$$


to obtain the full set of canonical variables

$$
\left\{q_{n}^{\mu}, p_{n}^{\mu} \mid n=0,1, \ldots ; \mu=0,1, \ldots, D\right\} .
$$

A natural choice of smearing space in flat space-time is the $\mathbb{R}^{D+1}$ valued sequences for which the canonical commutation relations (CCR's) still make sense, as suggested by the smearing formulae $q(f):=\sum_{n=0}^{\infty} f_{n}^{\mu} q_{n \mu}=\sum_{n=0}^{\infty} f_{n} \cdot q_{n}$, where $f_{n}^{\mu} \in \mathbb{R}$, and similar for $p(f)$, so:

$$
\begin{aligned}
{[p(f), q(h)] } & =\sum_{n, m=0}^{\infty} f_{n \mu} h_{m v}\left[p_{n}^{\mu}, q_{m}^{v}\right] \\
& =i f_{0} \cdot h_{0}+i \sum_{n=1}^{\infty} 2 \alpha^{\prime} n f_{n} \cdot h_{n}=: i\langle f, h\rangle_{0}^{\prime} .
\end{aligned}
$$

We choose a slightly smaller test-function space, i.e. $M^{D+1} \otimes \tilde{l}^{2}$, where

$$
\begin{aligned}
\tilde{l}^{2} & :=\left\{\left(f_{n}\right) \in l^{2} \mid f_{0}^{2}+2 \alpha^{\prime} \sum_{n=1}^{\infty} n f_{n}^{2}<\infty\right\} \\
& =\left\{\left(f_{n}\right\} \in l^{2} \mid \sum_{n=1}^{\infty} n f_{n}^{2}<\infty\right\}
\end{aligned}
$$

and the direct product is in terms of inner product spaces. Observe that $\tilde{l}^{2}$ is the Fourier transform of the Sobolev space $H^{1,2}$. Denote $S^{\prime}:=M^{D+1} \otimes \widetilde{l}^{2}$, $S_{0}^{\prime}:=M^{D+1} \otimes l_{0}, \quad S:=S^{\prime} \times S^{\prime} \cong \mathbb{R}^{2} \otimes M^{D+1} \otimes \tilde{l}^{2}, \quad$ and $\quad S_{0}:=S_{0}^{\prime} \times S_{0}^{\prime} \cong$ $\mathbb{R}^{2} \otimes M^{D+1} \otimes l_{0}$, where $l_{0}$ consists of sequences with only a finite number of nonzero entries, and $S^{\prime}$ comes equipped with the IIP $\langle\cdot, \cdot\rangle_{0}^{\prime}$. Then the field operator of this system is:

$$
\Phi(f)=\Phi\left(f^{(1)}, f^{(2)}\right):=p\left(f^{(1)}\right)+q\left(f^{(2)}\right)
$$

for all $f=\left(f^{(1)}, f^{(2)}\right) \in S=S^{\prime} \times S^{\prime}$, and the CCR's are

$$
\begin{aligned}
{[\Phi(f), \Phi(h)] } & =i\left\langle f^{(1)}, h^{(2)}\right\rangle_{0}^{\prime}-i\left\langle f^{(2)}, h^{(1)}\right\rangle_{0}^{\prime} \\
& =: i B(f, h) \quad \forall f, h \in S .
\end{aligned}
$$

That is, we obtain on $S$ the symplectic form:

$$
B(f, h):=f_{0}^{(1)} \cdot h_{0}^{(2)}-f_{0}^{(2)} \cdot h_{0}^{(1)}+2 \alpha^{\prime} \sum_{n=1}^{\infty} n\left(f_{n}^{(1)} \cdot h_{n}^{(2)}-f_{n}^{(2)} \cdot h_{n}^{(1)}\right) .
$$

It is possible to define on $S$ the CCR-algebra $\overline{\Delta(S, B)}$ cf. [9], which contains elements satisfying the exponentiated CCR's, but we leave this $C^{*}$-algebra approach for Sect. 6 .

Note that it is only possible to reconstruct the $p$ and $q$ operators (hence the $\alpha$ 's) out of the field operators $\Phi$ by the specification of the subspace $S^{\prime}$ in $S$ (for $f^{(1)}$ and $\left.f^{(2)}\right)$, and a different specification will result in different $p$ and $q$ operators. This specification is equivalent to the definition of a complex conjugation on $S$. Some information was lost in the transition to $\Phi$.

Next we examine the basic structures of $S=\mathbb{R}^{2} \otimes M^{D+1} \otimes \tilde{l}^{2}$. It is an IIPspace, where $\mathbb{R}^{2} \otimes \tilde{l}^{2}$ is a Hilbert space, and the indefinite part of the IIP comes from the Minkowski metric on $M^{D+1}$. Note that $S$ is a Hilbert space if we use the usual inner product of $\mathbb{R}^{D+1} \cong M^{D+1}$ instead of the Minkowski metric, and this 
specifies an important topology on $S$. So the IIP of $S$ is:

$$
\langle f, h\rangle_{0}:=\left\langle f^{(1)}, h^{(1)}\right\rangle_{0}^{\prime}+\left\langle f^{(2)}, h^{(2)}\right\rangle_{0}^{\prime} \quad \forall f, h \in S .
$$

The $\mathbb{R}^{2}$-part of $S$ carries the canonical "complex structure"

$$
K:=\left(\begin{array}{rr}
0 & 1 \\
-1 & 0
\end{array}\right) \otimes I \otimes I, \quad \text { so } K^{2}=-I,
$$

and $B(K f, K h)=B(f, h)$, but since $B(f, K f)$ is not positive for all $f \in S, K$ is not a complex structure in the usual sense of the word. Then the complex IIP on $S$ is:

$$
\langle f, h\rangle_{K}:=B(f, K h)+i B(f, h)=-\langle f, h\rangle_{0}+i B(f, h)
$$

for all $f, h \in S$. The complex IIP-space $\left\{S,\langle\cdot, \cdot\rangle_{K}\right\}$ is our chosen one-particle space on which a Fock construction will produce the representation space for the string. $K$ is canonical in the sense that it was obtained from input data, and $K$ provides precisely the information to reconstruct the $\alpha_{n}$ 's out of $\Phi(f)$. The complex IIP $\langle\cdot, \cdot\rangle_{K}$ is important in that it is Lorentz covariant. The one-particle space $S$ also is a Krein space because $M^{D+1}$ is: $M^{D+1}=M_{+} \oplus M_{-}$, where $M_{+}:=\left\{a \in M^{D+1} \mid\right.$ $\left.a_{0}=0\right\}$, and $M_{-}:=\left\{a \in M^{D+1} \mid a_{\mu}=0, \mu \neq 0\right\}$ and the Minkowski metric $g$ is positive (resp. negative) definite on $M_{+}$(resp. $M_{-}$), and $M_{ \pm}$are complete with respect to the real Hilbert space topology induced by $g$ on $M_{ \pm}$, i.e. it is a Krein space. Let $P_{ \pm}$be projections on $M_{ \pm}$respectively, then the fundamental symmetry is $J_{0}:=P_{+}-P_{-}, J_{0}^{2}=I$ i.e. $\left(J_{0} a\right)_{\mu}:=g_{\mu \mu} a_{\mu}$ for $a \in M^{D+1}$ (no summation) and $(a, b):=g\left(a, J_{0} b\right)$ for $a, b \in M^{D+1}$ defines the usual inner product of $\mathbb{R}^{D+1}$. Hence for $S=\mathbb{R}^{2} \otimes M^{D+1} \otimes \tilde{l}^{2}$ we have the decomposition $S=S_{+} \oplus S_{-}$with $S_{ \pm}:=\mathbb{R}^{2} \otimes M_{ \pm} \otimes \tilde{l}^{2}$ which makes it into a Krein space with Gram operator $I \otimes J_{0} \otimes I$ (or $J_{0}$ for short), and $\left[K, J_{0}\right]=0$. However in order to match with $\langle\cdot, \cdot\rangle_{K}$ above and obtain a positive definite inner product below, we choóse the fundamental symmetry $J=-J_{0}$ instead of $J_{0}$ above. Due to this $S$ becomes a complex Hilbert space with inner product

$$
\langle f, h\rangle_{\mathrm{K}}^{J}:=\langle f, J h\rangle_{\mathrm{K}}=B(f, K J h)+i B(f, J h)
$$

for all $f, h \in S$. With respect to the symplectic form $B(\cdot, J \cdot), K$ is indeed a complex structure in the usual sense. The Gram operator $J$ is not unique, because we can alter the decomposition $M_{+} \oplus M_{-}$through the addition of lightlike vectors to either component of the decomposition. However, via Bognar [4], we know that $\langle\cdot, \cdot\rangle_{K}$ is jointly continuous in the Hilbert space topology induced by $\langle\cdot, \cdot\rangle_{K}^{J}$, and that all Hilbert space topologies on $S$ are equivalent. Henceforth we denote adjoints w.r.t. $\langle\cdot, \cdot\rangle_{K}\left(\right.$ resp. $\left.\langle\cdot, \cdot\rangle_{K}^{J}\right)$ by ${ }^{+}$(resp. ${ }^{*}$ ), and take $J$ and $K$ fixed as above.

As convenience dictates, we can also decompose $S=\mathbb{R}^{2} \otimes M^{D+1} \otimes \tilde{l}^{2}$ further with relation to any of the spaces $\mathbb{R}^{2}=\mathbb{R} \oplus \mathbb{R}$ (real and imaginary parts), $M^{D+1}=\bigoplus_{\mu=0}^{D} \mathbb{R}_{(\mu)}$ (space-time components) and $\widetilde{l}^{2}=\bigoplus_{n=0}^{\infty} \mathbb{R}_{(n)}$ (excitation modes).

To conclude this section we collect some properties of operators on $S$, which we intend to use. First, note that $J^{+}=J^{*}=J^{-1}=J$, and that $A^{+}=J A^{*} J$ for an operator $A$ on $S$. Call an operator $A: S \mapsto S$ with dense domain $D(A)$, J-symmetric (resp. J-unitary) if $\langle f, A h\rangle_{\mathrm{K}}=\langle A f, h\rangle_{\mathrm{K}} \quad$ (resp. $\langle f, h\rangle_{\mathrm{K}}=\langle A f, A h\rangle_{\mathrm{K}}$ ) for all $f, h \in D(A)$. Then all $\mathrm{J}$-symmetric and all J-unitary operators are closable, and each $\mathrm{J}$-unitary operator has a J-unitary inverse [5]. 


\section{Construction of the String Operators}

In this section we define the operators $\Phi(f), L_{n}$ on the Fock space constructed from $S$, examine domain questions and verify commutation relations. This follows the construction of Mintchev [5] on IIP-space from which we use results freely.

Starting from the complex Hilbert space $\left\{S,\langle\cdot, \cdot\rangle_{J}^{K}\right\}=:\left\{\mathscr{H}^{(1)},\langle\cdot, \cdot\rangle_{(1)}\right\}$, define the symmetric Fock-space $\mathscr{F}(S):=\bigoplus_{n=0}^{\infty} \mathscr{H}^{(n)}$, where $\mathscr{H}^{(0)}=\mathbb{C}, \mathscr{H}^{(n)}=$ $\sigma_{n} \otimes_{k=1}^{n} \mathscr{H}_{k}^{(1)}, \mathscr{H}_{k}^{(1)}=\mathscr{H}^{(1)}=S$ and $\sigma_{n}:=\frac{1}{n !} \sum_{p \in S_{n}} p$ is the symmetrization operator on $n$ indices. The Fock inner product $\langle\cdot, \cdot\rangle$ arises from $\langle\cdot, \cdot\rangle_{(1)}=\langle\cdot, \cdot\rangle_{J}^{K}$, and since the set of decomposable vectors

$$
V^{(n)}=\left\{\sigma_{n}\left(f_{1} \otimes \ldots \otimes f_{n}\right) \mid f_{i} \in \mathscr{H}^{(1)}, i=1, \ldots, n\right\}
$$

generates a dense subspace of $\mathscr{H}^{(n)}$, it suffices for an operator to specify it on these. Also, the various decompositions of $S$ give decompositions of $\mathscr{F}(S)$ by $\mathscr{F}\left(S_{a} \oplus S_{b}\right) \cong \mathscr{F}\left(S_{a}\right) \otimes \mathscr{F}\left(S_{b}\right)$. Define $\Gamma(J) \in \mathscr{B}(\mathscr{F}(S))$ by $\Gamma(J) \mid V^{(n)}=\bigotimes_{k=1}^{n} J$, then the distinguished IIP of $\mathscr{F}(S)$ is:

$$
\langle f, h\rangle_{J}:=\langle f, \Gamma(J) h\rangle \quad \forall f, h \in \mathscr{F}(S) .
$$

Note that on $\mathscr{H}^{(1)}=S,\langle\cdot, \cdot\rangle_{J}$ is just $\langle\cdot, \cdot\rangle_{K}$. Then $[\Gamma(J)]^{2}=1$ so $\{\mathscr{F}(S)$, $\left.\langle\cdot, \cdot\rangle_{J}\right\}$ is a Krein space hence $\langle\cdot, \cdot\rangle_{J}$ is jointly continuous with respect to Fock-Hilbert space topology (cf. [5] Prop. 4). Let $a^{+}(f)=a^{*}(f): V^{(n)} \mapsto V^{(n+1)}$ be the usual creation operator:

$$
a^{+}\left(h_{0}\right)\left(\sigma_{n} h_{1} \otimes h_{2} \otimes \cdots \otimes h_{n}\right):=\sqrt{n+1} \sigma_{n+1} h_{0} \otimes h_{1} \otimes h_{2} \otimes \cdots \otimes h_{n}
$$

for all $h_{i} \in \mathscr{H}^{(1)}$. Define $a^{-}(f): V^{(n)} \mapsto V^{(n-1)}$ by:

$$
a^{-}(f)\left(\sigma_{n} h_{1} \otimes \cdots \otimes h_{n}\right):=\sqrt{n} \sigma_{n}\left\langle f, h_{1}\right\rangle_{J} h_{2} \otimes \cdots \otimes h_{n}
$$

for all $f, h_{i} \in \mathscr{H}^{(1)}$, where $\sigma_{n}$ acts on the indices of all the $h_{i}$ 's following it. Then $a^{+}$ and $a^{-}$are $\langle\cdot, \cdot\rangle_{J}$-adjoints of one another, and

$$
a^{-}(f)=\Gamma(J) a(f) \Gamma(J)=a(J f),
$$

where $a(f)$ is the usual annihilator. Let $F_{0} \subset \mathscr{F}(S)$ be the (dense) finite particle subspace, then $a^{ \pm}(f)$ can be extended by linearity to $F_{0}$.

Then $\left[a^{-}(h), a^{+}(f)\right] \cdot \chi=\left[a(J h), a^{*}(f)\right] \cdot \chi=\langle J h, f\rangle_{K}^{J} \cdot \chi=\langle h, f\rangle_{J} \cdot \chi \forall \chi \in F_{0}$; $h, f \in S$. Define the field operator:

$$
\Phi(f):=\frac{1}{\sqrt{2}}\left(a^{+}(f)+a^{-}(f)\right)=\frac{1}{\sqrt{2}}\left(a^{*}(f)+a(J f)\right)=p\left(f^{(1)}\right)+q\left(f^{(2)}\right)
$$

for all $f \in S$, where the last equality follows from

$$
p\left(f^{(1)}\right):=\frac{i}{\sqrt{2}}\left(a^{+}\left(f^{(1)}\right)-a^{-}\left(f^{(1)}\right)\right), \quad q\left(f^{(2)}\right):=\frac{1}{\sqrt{2}}\left(a^{+}\left(f^{(2)}\right)+a^{-}\left(f^{(2)}\right)\right),
$$

where $f^{(1)} \in S^{(1)}=\left(\begin{array}{l}1 \\ 0\end{array}\right) \otimes M^{D+1} \otimes \tilde{l}^{2}$ and $f^{(2)} \in S^{(2)}=\left(\begin{array}{l}0 \\ 1\end{array}\right) \otimes M^{D+1} \otimes \tilde{l}^{2}$, employing the definition of $K$ and the fact that $a(f)$ is conjugate linear. The relation of 
$\Phi(f)$ to the usual field operator $\Psi(f)=\frac{1}{\sqrt{2}}\left(a^{*}(f)+a(f)\right)$ is easily found:

$$
\Psi(f)-\Phi(f)=\frac{1}{\sqrt{2}} a(f-J f)=\sqrt{2} a\left(P_{+} f\right),
$$

so using $a(f)=\frac{1}{\sqrt{2}}(\Psi(f)+K \Psi(K f))$, we see

$$
\Phi(f)=\Psi\left(P_{-} f\right)-K \Psi\left(K P_{+} f\right)
$$

Then we have (cf. [5] Theorem 1, 2):

(a) $\Phi(f)$ is closable for all $f \in S$.

(b) $F_{0}$ is a set of analytic vectors for $\Phi(f)$.

(c) Given a sequence $\left\{f_{k}\right\} \subset S$, s- $\lim _{k \rightarrow \infty} f_{k}=f$, we have

$$
\operatorname{s-lim}_{k \rightarrow \infty} \Phi\left(f_{k}\right) \chi=\Phi(f) \chi \quad \forall \chi \in F_{0} .
$$

(Notation: s-lim means limit in the strong Hilbert-space topology)

(d) The vacuum $\Omega=(1,0,0, \ldots) \in F_{0}$ is cyclic for the set $\{\Phi(f) \mid f \in S\}$.

(e) $[\Phi(f), \Phi(h)] \chi=i \operatorname{Im}\langle f, h\rangle_{J} \cdot \chi=i B(f, h) \chi$ for all $\chi \in F_{0}$ and for all $f, h \in S$.

(f) Define

$$
W(f) \chi:=\operatorname{sim}_{k \rightarrow \infty} \sum_{k=0}^{K} \frac{[i \Phi(f)]^{k}}{k !} \chi \quad \forall \chi \in F_{0},
$$

then $W(f)$ is closable for all $f \in S$.

(g) $\bar{W}(f+h) \chi=\exp \left(\frac{i}{2} B(f, h)\right) \bar{W}(f) \bar{W}(h) \chi \forall f, h \in S$ and $\forall \chi \in F_{0}$.

(h) $W(f)$ is a $\langle\cdot, \cdot\rangle_{J}$-unitary operator on $F_{0}$ for all $f \in S$.

Furthermore by [5], Prop. 6, given a $\langle\cdot, \cdot\rangle_{J}$-unitary operator $U$ on $S$ with domain and range $D(U)$ and $R(U)$, define

$$
\begin{aligned}
D^{n}(\Gamma(U)) & :=\operatorname{Span}\left\{\sigma_{n}(D(U) \otimes \cdots \otimes D(U))\right\} \subset V^{(n)}, \\
D^{n}\left(\Gamma\left(U^{-1}\right)\right) & :=\operatorname{Span}\left\{\sigma_{n}(R(U) \otimes \cdots \otimes R(U))\right\} \subset V^{(n)}, \\
F_{0}^{R} & :=\left\{\chi \in F_{0} \mid \chi^{(k)} \in D^{k}\left(\Gamma\left(U^{-1}\right)\right)\right\} .
\end{aligned}
$$

Then for all $f \in D(U)$ and $\chi \in F_{0}^{R}$,

$$
\Gamma(U) \Phi(f) \Gamma\left(U^{-1}\right) \chi=\Phi(U f) \chi,
$$

where $\Gamma(U)$ is defined as usual with $D^{n}(\Gamma(U))$ in the domain of $\Gamma(U) \mid V^{(n)}$.

With all this preparation behind us, it is now possible to define the $\alpha$ operators and to construct $L_{n}, n \in \mathbb{Z}$. Define $\delta(n, \mu) \in S=\mathbb{R}^{2} \otimes M^{D+1} \otimes \tilde{l}^{2}$ by $\delta(n, \mu)=$ $\left(\begin{array}{l}0 \\ 1\end{array}\right) \otimes e_{\mu} \otimes \varepsilon_{n}$, where $e_{\mu}$ (resp. $\left.\varepsilon_{n}\right)$ is the $\mu^{\text {th }}$ (resp. $n^{\text {th }}$ ) unit vector of $M^{D+1}$ (resp. $l^{2}$ ). 
Then we make the following identifications with the heuristic objects:

$$
\begin{aligned}
\alpha_{n}^{\mu} & =a^{-}(\delta(n, \mu)), \quad n \geqq 1, \\
\left(\alpha_{n}^{\mu}\right)^{*} & =\alpha_{-n}^{\mu}=a^{+}(\delta(n, \mu)), \quad n \geqq 1 ; \\
q_{0}^{\mu} & =q^{\mu}=\frac{1}{\sqrt{2}}\left(a^{+}(\delta(0, \mu))+a^{-}(\delta(0, \mu))\right) ; \\
p_{0}^{\mu} & =p^{\mu}=\frac{i}{\sqrt{2}}\left(a^{+}(\delta(0, \mu))-a^{-}(\delta(0, \mu))\right),
\end{aligned}
$$

and so $\left[\alpha_{n}^{\mu}, \alpha_{-m}^{v}\right] \chi=\left[a^{-}(\delta(n, \mu)), a^{+}(\delta(m, v))\right] \chi=\langle\delta(m, v), \delta(n, \mu)\rangle_{J} \cdot \chi=$ $-2 \alpha^{\prime} n g^{\mu v} \delta_{n-m, 0}$ for $n, m>0$ as expected from the construction. Moreover, $\alpha_{n}^{\mu} \Omega=0 \forall n \geqq 1$ as in the heuristic theory. These commutation relations agree with the heuristic ones. With these identifications we can now use the heuristic expressions:

$$
\begin{gathered}
L_{0}=\frac{1}{2 \alpha^{\prime}} \sum_{k=1}^{\infty} \alpha_{-k} \cdot \alpha_{k}+\alpha^{\prime} p \cdot p \text { and } \\
L_{n}=\frac{1}{2 \alpha^{\prime}} \sum_{k=1}^{\infty} \alpha_{-k} \cdot \alpha_{n+k}+\frac{1}{4 \alpha^{\prime}} \sum_{k=1}^{n-1} \alpha_{k} \cdot \alpha_{n-k}+\alpha_{n} \cdot p
\end{gathered}
$$

for $n \geqq 1$, and $L_{-n}=\left(L_{n}\right)^{+}$to define the Virasoro operators and we need to determine the domains on which the infinite sums converge for these expressions to make sense. We know that all finite partial sums in these expressions preserve $F_{0}$.

Lemma 3.1. $F_{0}$ is not the domain of $L_{n}, n \geqq 0$.

Proof. We exhibit a vector $\chi \in F_{0}$ for which $L_{0} \chi \notin \mathscr{F}(S)$, and the case for $L_{n} \chi$ for $n>0$ follows similarly. Consider the vector $a^{+}(f) \Omega \in F_{0}$ with $f=\left(\begin{array}{l}0 \\ 1\end{array}\right) \otimes e_{0} \otimes\left(f_{n}\right) \in S=\mathbb{R}^{2} \otimes M^{D+1} \otimes \tilde{l}^{2}$, where $\left(f_{n}\right) \in \tilde{l}^{2}:=\left\{\left(h_{n}\right) \in l^{2} \mid \sum_{n=1}^{\infty} n h_{n}^{2}\right.$ $<\infty\}$ is given by $f_{n}=n^{-(r+1)}$ with $0<r<1$. (Then clearly $\sum_{n=1}^{\infty} n f_{n}^{2}=$ $\sum_{n=1}^{\infty} n^{-(2 r+1)}<\infty$ for $r>0$.) Then

$$
\begin{aligned}
L_{0} a^{+}(f) \Omega & =\sum_{k=1}^{\infty} \alpha_{-k} \cdot \alpha_{k} a^{+}(f) \Omega \\
& =\sum_{\mu=0}^{D} \sum_{k=1}^{\infty} a^{+}(\delta(k, \mu)) g_{\mu \mu} a^{-}(\delta(k, \mu)) a^{+}(f) \Omega \\
& =-\sum_{k=1}^{\infty} a^{+}(\delta(k, 0)) a^{-}(\delta(k, 0)) a^{+}(f) \Omega \\
& =\sum_{k=1}^{\infty} a^{+}(\delta(k, 0)) 2 k \alpha^{\prime} f_{k}^{0} \Omega=2 \alpha^{\prime} a^{+}(\tilde{f}) \Omega,
\end{aligned}
$$

where $\tilde{f}=\left(\begin{array}{l}0 \\ 1\end{array}\right) \otimes e_{0} \otimes\left(n f_{n}\right)$ and we used

$$
\left[a^{-}(\delta(k, 0)), a^{+}(f)\right]=\langle\delta(k, 0), f\rangle_{J}=-2 \alpha^{\prime} k f_{k}^{0} .
$$


However $\left(n f_{n}\right) \notin \tilde{l}^{2}$ because $\sum_{n=1}^{\infty} n\left(n f_{n}\right)^{2}=\sum_{n} n^{1-2 r}$ diverges for $r<1$ which we have here. Hence $a^{+}(\tilde{f}) \Omega \notin \mathscr{F}(S)$.

Recall that $S_{0}:=\mathbb{R}^{2} \otimes M^{D+1} \otimes l_{0}$ (the finite mode space) is dense in $S$ with respect to the Hilbert-space topology. Then the subspace of finite particle vectors constructed on $S_{0}$ :

$$
F_{00}:=\operatorname{Span}\left\{a^{+}\left(f_{1}\right) \ldots a^{+}\left(f_{n}\right) \Omega \mid f_{i} \in S_{0}, n<\infty\right\} \subset F_{0}
$$

is also dense in $\mathscr{F}(S)$ because the tensor product topology restricted to each component is just the original Hilbert space topology, and $\Omega \in F_{00}$.

Lemma 3.2. $F_{00}$ is in the domain of all $L_{n}, n \in \mathbb{Z}$, and is also preserved by these.

Proof. This follows easily from the observation that for $\chi \in F_{00}$ only a finite number of terms in the sums $\sum_{k=1}^{\infty} \alpha_{-k} \cdot \alpha_{n+k} \chi, n \geqq 0$ can be nonzero, so $L_{n} \chi \in F_{00}$ $\forall \chi \in F_{00}, n \geqq 0 . L_{-n} \chi \in F_{00}$ follows similarly.

The Virasoro relations on $F_{00}$ :

$$
\left[L_{n}, L_{m}\right] \chi=(n-m) L_{n+m} \chi+\frac{D+1}{12} n\left(n^{2}-1\right) \delta_{n+m, 0} \chi \quad \forall \chi \in F_{00}
$$

are proven in complete analogy with the heuristic derivation, simply from the CCR's and that $L_{n}$ preserves $F_{00}$ for all $n$. The indirect methods of the heuristic approaches [1] are unnecessary, elementary (but tedious) algebra is enough.

This setting up of the operator string theory is concluded by restricting the set of field operators $\{\Phi(f) \mid f \in S\}$, since for $f \in S \backslash S_{0}, \Phi(f)$ will not in general preserve $F_{00}$. However we know that for a sequence $\left\{f_{k}\right\} \subset S_{0}$, s- $\lim _{k \rightarrow \infty} f_{k}=f \in S \backslash S_{0}$, that s- $\lim _{k \rightarrow \infty} \Phi\left(f_{k}\right) \chi=\Phi(f) \chi \in F_{0}$ for all $\chi \in F_{00}$, and that for $h \in S_{0}, \Phi(h)$ preserves $F_{00}$. Hence since $S_{0}$ is dense in $S$, the specification of $\left\{\Phi(h) \mid h \in S_{0}\right\}$ on $F_{00}$ will uniquely determine $\{\Phi(h) \mid h \in S\}$ on $F_{0}$. No information is lost by restricting our attention to the set $\left\{\Phi(h) \mid h \in S_{0}\right\}$, which we henceforth do.

At this point we have properly defined all the basic spaces and operators occurring in the heuristic model, and these have the correct commutation relations. To summarize, we have:

1. A one particle Krein space $\left\{S,\langle\cdot, \cdot\rangle_{K}, J\right\}$ with dense subspace $S_{0}$,

2. A Fock-Krein space $\mathscr{F}(S), \Gamma(J)$, with dense subspace $F_{00}$ containing the vector $\Omega$,

3. A set of field operators $\left\{\Phi(f) \mid f \in S_{0}\right\}$ preserving $F_{00}$, and making $\Omega$ algebraically cyclic for $F_{00}$.

4. A set of string constraints $\left\{L_{n} \mid n \in \mathbb{Z}\right\}$ preserving $F_{00}$, and on $F_{00}$ these have the requisite commutation relations with themselves and with $\left\{\Phi(f) \mid f \in S_{0}\right\}$.

So at an infinitesimal level, the string operators are well-defined in the FockKrein representation. In the following sections we want to see whether this infinitesimal theory can be integrated up to a theory of bounded operators, which is necessary preparation for a $\mathrm{C}^{*}$-algebra expression of the string. This Fock-Krein representation is the one suggested by heuristic string theory [12], where the IIP is traced solely to the occurrence of $g^{\mu \nu}$ in the commutation relations of the mode oscillators, and it involved no mixing between different modes. That is why we chose a fundamental symmetry $J$ of $M^{D+1}$ instead of a more general fundamental symmetry on $M^{D+1} \otimes \tilde{l}^{2}$. 
It is necessary to remark at this point that there is another expression of the string operators. Recall that $\mathscr{F}\left(\mathbb{C}^{n}\right) \cong L^{2}\left(\mathbb{C}^{n}, \mu\right)$, where $\mu$ is the Gaussian measure and the Fock representation corresponds to a harmonic oscillator representation (using Hermite polynomials) on $L^{2}\left(\mathbb{C}^{n}, \mu\right)$, the vacuum being $\Omega=1$, cf. [20]. Hence on decomposition of $S$ into mode spaces, we obtain for the Fock representation an infinite tensor product of these representations, and so $L_{0}$ which preserves modes, can be written purely as a differential operator on $L^{2}\left(\mathbb{C}^{D+1}, \mu\right)$. In fact, this turns out to be a Klein-Gordon equation, cf. [21]. The operators $L_{n}, n \neq 0$ in this formulation consist of a combination of differential and mode shifting operators, $\mathrm{cf}$. [21]. Since the solutions of a Klein Gordon equation are distributions concentrated on a mass hyperboloid, it is clear that the constraint $L_{0} \psi=\alpha(0) \psi$ cannot have a solution $\psi$ in the Hilbert space.

In view of the fact that the constraints $L_{n}$ are sums of terms of the form $a^{ \pm}(f) \cdot a^{ \pm}(h)$, we will find the following lemma useful.

Lemma 3.3. $\left[a^{ \pm}(f) \cdot a^{ \pm}(h), \Gamma(J)\right] \chi=0 \forall \chi \in F_{0}, f, h \in S$.

Proof. $J$ is a unitary operator on the Hilbert space $\left\{S,\langle\cdot, \cdot\rangle_{J}^{K}\right\}$, and hence $a(J f) \chi=\Gamma(J) a(f) \Gamma(J) \chi \quad \forall \chi \in F_{0}, \quad f \in S$ and likewise for $a^{*}(J f)$, using $\Gamma(J)^{-1}=\Gamma(J)$. Now $a^{+}(f)=a^{*}(f)$ and $a^{-}(f)=a(J f)$, hence

$$
a^{ \pm}(J f) \chi=\Gamma(J) a^{ \pm}(f) \Gamma(J) \chi \quad \forall f \in S, \chi \in F_{0},
$$

so for $a^{ \pm}(f) \cdot a^{ \pm}(h)=\sum_{k=1}^{D} a^{ \pm}\left(f_{k}\right) a^{ \pm}\left(h_{k}\right)-a^{ \pm}\left(f_{0}\right) a^{ \pm}\left(h_{0}\right)$,

$$
\begin{aligned}
\Gamma(J) a^{ \pm}(f) \cdot a^{ \pm}(h) \Gamma(J) \chi & =\Gamma(J) a^{ \pm}(f) \Gamma(J) \cdot \Gamma(J) a^{ \pm}(h) \Gamma(J) \chi \\
& =a^{ \pm}(J f) \cdot a^{ \pm}(J h) \chi .
\end{aligned}
$$

Now $J f_{0}=f_{0}$ and $J f_{k}=-f_{k}$, where $f_{0}$ is the time component and $f_{k}$ are the space components of $f$. Hence

$$
\begin{aligned}
a^{ \pm}(J f) \cdot a^{ \pm}(J h) \chi & =\sum_{k=1}^{D} a^{ \pm}\left(J f_{k}\right) a^{ \pm}\left(J h_{k}\right) \chi-a^{ \pm}\left(J f_{0}\right) a^{ \pm}\left(J h_{0}\right) \chi \\
& =\sum_{k=1}^{D} a^{ \pm}\left(-f_{k}\right) a^{ \pm}\left(-h_{k}\right) \chi-a^{ \pm}\left(f_{0}\right) a^{ \pm}\left(h_{0}\right) \chi \\
& =a^{ \pm}(f) \cdot a^{ \pm}(h) \chi,
\end{aligned}
$$

and so $\left[a^{ \pm}(f) \cdot a^{ \pm}(h), \Gamma(J)\right] \chi=0$.

Since the heuristic string is only given at the infinitesimal level, we intend in the next sections to integrate up the gauge and time translations, in order to define the string as a $C^{*}$-algebra $(\overline{\Delta(S, B)}$ in fact) together with the appropriate automorphism groups. We also intend to prove J-unitary implementability of these in the given Fock-Krein representation, which is an interesting question in its own right.

\section{Time Evolution: Integration and Implementation}

In this section we write the infinitesimal time translations as infinitesimally symplectic transformations on $(S, B)$, integrate it up to a symplectic one parameter 
group and show that this group is implementable in the Fock-Krein representation by a strong operator continuous one parameter group.

The time evolution of the string is defined with respect to an internal proper time parameter, determined by the choice of a parametrization for the world-sheet of the string, and this is why the use of the Hamiltonian framework here does not spoil the Poincare covariance of the string. The Hamiltonian $H=L_{0}$ generates the infinitesimal time translations.

In $(\mathrm{A} .1,2)$ of the appendix we calculated:

$$
\left(\operatorname{ad} i \lambda L_{0}\right)(\Phi(f)) \chi=\Phi\left(\lambda T_{0} f\right) \chi \quad \forall \chi \in F_{00}, \lambda \in \mathbb{R}, f \in S_{0},
$$

with

$$
\begin{aligned}
& \left(T_{0} f\right)^{(1)}=\left(-2 \alpha^{\prime} f_{0}^{(2)},-f_{1}^{(2)},-2 f_{2}^{(2)},-3 f_{3}^{(2)}, \ldots\right) \\
& \left(T_{0} f\right)^{(2)}=\left(0, f_{1}^{(1)}, 2 f_{2}^{(1)}, 3 f_{3}^{(1)}, \ldots\right),
\end{aligned}
$$

and because of the good growth properties of $T_{0} f$, the exponential series:

$$
\begin{aligned}
\left(\exp \lambda T_{0}\right)(f):= & \text { s-lim } \sum_{N \rightarrow \infty} \frac{\left(\lambda T_{0}\right)^{k}(f)}{k !} \text { will converge absolutely: } \\
& \left(T_{0}^{2 k} f\right)^{(i)}=(-1)^{k}\left(0, f_{1}^{(i)}, 2^{2 k} f_{2}^{(i)}, 3^{2 k} f_{3}^{(i)}, \ldots\right), \\
& \left(T_{0}^{2 k+1} f\right)^{(1)}=(-1)^{k+1}\left(0, f_{1}^{(2)}, 2^{2 k+1} f_{2}^{(2)}, 3^{2 k+1} f_{3}^{(2)}, \ldots\right), \\
& \left(T_{0}^{2 k+1} f\right)^{(2)}=(-1)^{k}\left(0, f_{1}^{(1)}, 2^{2 k+1} f_{2}^{(1)}, 3^{2 k+1} f_{3}^{(1)}, \ldots\right),
\end{aligned}
$$

where $i=1,2$ and $k \geqq 1$ above. So

$$
\left|\frac{\left(\lambda T_{0}\right)^{k}(f)_{n}^{(i)}}{k !}\right| \leqq \frac{(\lambda n)^{k}}{k !}\left(\left|f_{n}^{(1)}\right|+\left|f_{n}^{(2)}\right|\right) \underset{\infty}{\stackrel{k}{\rightarrow} 0,}
$$

and this convergence is fast enough for the series to converge absolutely. A trivial calculation then produces the result:

$$
\begin{aligned}
& \left(\left(\exp \lambda T_{0}\right) f\right)_{0}^{(1)}=f_{0}^{(1)}-2 \alpha^{\prime} f_{0}^{(2)} \lambda, \\
& \left(\left(\exp \lambda T_{0}\right) f\right)_{0}^{(2)}=f_{0}^{(2)}, \\
& \left(\left(\exp \lambda T_{0}\right) f\right)_{n}^{(1)}=f_{n}^{(1)} \cos \lambda n-f_{n}^{(2)} \sin \lambda n, \quad n \geqq 1 \\
& \left(\left(\exp \lambda T_{0}\right) f\right)_{n}^{(2)}=f_{n}^{(2)} \cos \lambda n+f_{n}^{(1)} \sin \lambda n, \quad n \geqq 1 .
\end{aligned}
$$

This constitutes a linear motion for the centre of mass variables, and a rotation for each excitation mode separately, which is what we expect of a reasonable time evolution for a free string. Note that $\exp \lambda T_{0}$ is extendible from $S_{0}$ to all of $S$. Observe at this point that the integration $\exp \lambda T_{0}$ of the infinitesimal time evolution $T_{0}$ has the following consequence. If the quantum theory provides us with a Lie algebra homomorphism from $\mathrm{LDiff}^{+} S^{1}$ to the infinitesimal gauge transformations such that $T_{0}$ is the image of the generator of the rotations on the circle, then it is not possible to integrate this homomorphism up to the corresponding groups. This is because the rotation group is not simply connected, but $\exp \lambda T_{0}$ produces the simply connected group $\mathbb{R}$ on the centre of mass variable. At the best we will have to use some covering group of $\operatorname{Diff}^{+} S^{1}$. For $\exp \lambda T_{0}$ to be an acceptable time translation, it must be symplectic. 
Lemma 4.1. $B\left(e^{\lambda T_{0}} f, e^{\lambda T_{0}} h\right)=B(f, h) \forall f, h \in S$.

Proof. This is a trivial calculation:

$$
\begin{aligned}
B\left(e^{\lambda T_{0}} f, e^{\lambda T_{0}} h\right)= & \left(f_{0}^{(1)}-2 \alpha^{\prime} \lambda f_{0}^{(2)}\right) \cdot h_{0}^{(2)}-f_{0}^{(2)} \cdot\left(h_{0}^{(1)}-2 \alpha^{\prime} \lambda h_{0}^{(2)}\right) \\
& +2 \alpha^{\prime} \sum_{n=1}^{\infty} n\left[\left(f_{n}^{(1)} \cos \lambda n-f_{n}^{(2)} \sin \lambda n\right) \cdot\left(h_{n}^{(2)} \cos \lambda n+h_{n}^{(1)} \sin \lambda n\right)\right. \\
& \left.-\left(f_{n}^{(2)} \cos \lambda n+f_{n}^{(1)} \sin \lambda n\right) \cdot\left(h_{n}^{(1)} \cos \lambda n-h_{n}^{(2)} \sin \lambda n\right)\right] \\
= & f_{0}^{(1)} \cdot h_{0}^{(2)}-f_{0}^{(2)} \cdot h_{0}^{(1)}+2 \alpha^{\prime} \sum_{n=1}^{\infty} n\left(f_{n}^{(1)} \cdot h_{n}^{(2)}-f_{n}^{(2)} \cdot h_{n}^{(1)}\right) \\
= & B(f, h) \quad \forall f, h \in S .
\end{aligned}
$$

Having obtained a reasonable one-parameter symplectic group $e^{\lambda T_{0}}$ on $S$ for the time evolution, we now need to examine its implementability (with J-unitary operators) in the Fock-Krein representation above. The formal equation used by physicists:

$$
\left(\operatorname{Ad} \exp i \lambda L_{0}\right) \Phi(f)=\left(\exp \operatorname{ad} i \lambda L_{0}\right) \Phi(f)=\Phi\left(e^{\lambda T_{0}} f\right)
$$

suggests that we try to define exp $i \lambda L_{0}$ as a J-unitary implementer of $e^{\lambda T_{0}}$. However the physicist's equation originates from Lie group theory analogies, and for operators on infinite dimensional Hilbert space it need not hold. In fact, due to the kinetic term $p \cdot p$ of $L_{0}$ we know that $\sum_{k=0}^{\infty}(k !)^{-1}(i \lambda p \cdot p)^{k} \chi$ cannot converge for all $\lambda \in \mathbb{R}, \chi \in F_{00}$ in Hilbert space topology, a fact we show in the next lemma.

Lemma 4.2. There are $\chi \in F_{00}$ and $\lambda \in \mathbb{R}$ for which the sequence $\sum_{k=0}^{N} \frac{\left(i \lambda L_{0}\right)^{k}}{k !} \chi d i$ verges with $N$ for the Hilbert space topology of $\mathscr{F}(S)$.

Proof. We will use the fact that a series $\sum u_{n}$ diverges if the sequence $\left\{u_{n}\right\}$ does not converge to zero. Choose $\chi=\Omega \in F_{00}$, then

$$
L_{0} \Omega=\left(\frac{1}{2 \alpha^{\prime}} \sum_{k=1}^{\infty} \alpha_{-k} \cdot \alpha_{k}+\alpha^{\prime} p \cdot p\right) \Omega=\alpha^{\prime} p \cdot p \Omega,
$$

and so since $\left[\alpha_{-k} \cdot \alpha_{k}, p \cdot p\right]=0 \forall k \geqq 1$, we have $\left(L_{0}\right)^{n} \Omega=\left(\alpha^{\prime} p \cdot p\right)^{n} \Omega$. Using $p_{\mu}=\frac{i}{\sqrt{2}}\left(a^{+}(\delta(0, \mu))-a^{-}(\delta(0, \mu))\right)$, we find $(p \cdot p)^{n} \Omega=\left(\frac{-1}{2}\right)^{n}\left(a_{0}^{+} \cdot a_{0}^{+}\right)^{n} \Omega+\zeta$, where $a_{0}^{+} \cdot a_{0}^{+}:=g_{\mu \nu} a^{+}(\delta(0, \mu)) a^{+}(\delta(0, v))$ and $\zeta \perp\left(a_{0}^{+} \cdot a_{0}^{+}\right)^{n} \Omega$ because they belong to different particle spaces. (Clearly $\left(a_{0}^{+} \cdot a_{0}^{+}\right)^{n} \Omega \in \mathscr{H}^{(2 n)}$ and $\zeta$ is a linear combination of vectors belonging to particle spaces $\mathscr{H}^{(k)}$ with $k<2 n$.) Because of this orthogonality, $\left\|(p \cdot p)^{n} \Omega\right\| \geqq \frac{1}{2^{n}}\left\|\left(a_{0}^{+} \cdot a_{0}^{+}\right)^{n} \Omega\right\|$. Write $a_{0}^{+} \cdot a_{0}^{+}=$ $-a^{+}(\delta(0,0)) a^{+}(\delta(0,0))+\sum_{i=1}^{D} a^{+}(\delta(0, i)) a^{+}(\delta(0, i))$. Then due to the contribution of $\tilde{l}^{2}$ to the inner product of $S$, for any component $i, a_{0 i}^{+} a_{0 i}^{+} \Omega \perp a_{0 j}^{+} a_{0 j}^{+} \Omega$ when $i \neq j$ and where $a_{0 i}^{+}:=a^{+}(\delta(0, i))$. Hence as above for $(p \cdot p)^{n} \Omega$, we can 
write $\left(a_{0}^{+} \cdot a_{0}^{+}\right)^{n} \Omega=\left(a_{0 i}^{+} a_{0 i}^{+}\right)^{n} \Omega+\xi$, where $\xi \perp\left(a_{0 i}^{+}\right)^{2 n} \Omega$ and so $\left\|\left(a_{0}^{+} \cdot a_{0}^{+}\right)^{n} \Omega\right\|$ $\geqq\left\|\left(a_{0 i}^{+}\right)^{2 n} \Omega\right\|$. By definition of $a_{0}^{+}, \quad\left(a_{0 i}^{+}\right)^{2 n} \Omega=\sqrt{(2 n) !} \delta(0, i) \otimes \ldots \otimes \delta(0, i)$ (2n times) so $\left\|\left(a_{0 i}^{+}\right)^{2 n} \Omega\right\|=\sqrt{(2 n) !}$ and hence $\left\|L_{0}^{n} \Omega\right\| \geqq\left|\frac{\alpha^{\prime}}{2}\right|^{n} \sqrt{(2 n) !}$, i.e.

$$
\begin{aligned}
\left\|(k !)^{-1}\left(i \lambda L_{0}\right)^{k} \Omega\right\| & \geqq\left|\frac{\lambda \alpha^{\prime}}{2}\right|^{k}(k !)^{-1} \sqrt{(2 k) !}=\left|\frac{\lambda \alpha^{\prime}}{2}\right|^{k}\left[\frac{2 k(2 k-1) \ldots(k+1)}{k(k-1) \ldots 2 \cdot 1}\right]^{1 / 2} \\
& =\left|\frac{\lambda \alpha^{\prime}}{2}\right|^{k}\left[2\left(2+\frac{1}{k-1}\right)\left(2+\frac{2}{k-2}\right) \ldots\left(2+\frac{k-1}{k-(k-1)}\right)\right]^{1 / 2} \\
& \geqq\left|\lambda \alpha^{\prime} \sqrt{2}\right|^{k},
\end{aligned}
$$

and this will not converge to zero with $k \rightarrow \infty$ if $|\lambda|>1 / \alpha^{\prime} \sqrt{2}$.

Hence s-lim $\lim _{N \rightarrow \infty} \sum_{k=0}^{N} \frac{i \lambda L_{0}}{k !} \chi$ does not define a suitable implementer for $e^{\lambda T_{0}}$. We now aim to find a suitable implementer.

Recall that the J-inner product of $S$ which defines the IIP of $\mathscr{F}(S)$ is $\langle f, h\rangle_{K}:=$ $B(f, K h)+i B(f, h)$, and a bounded operator $V$ on $S$ is J-unitary if $\langle V f, V h\rangle_{K}=\langle f, h\rangle_{K} \forall f, h \in S$. From the imaginary part of $\langle\cdot, \cdot\rangle_{K}$ we see that this means that $V$ is symplectic, and this in turn implies via the real part that $[K, V]=0$.

Lemma 4.3. $\exp \lambda T_{0}$ is not $J$-unitary on $S$. Let $P$ be the projection onto the centre of mass variables of $S$. Then $\left[P, e^{\lambda T_{0}}\right]=0$ and $C_{\lambda T_{0}}:=P e^{\lambda T_{0}}+(1-P)$ is not $J$ unitary, but $X_{\lambda T_{0}}:=P+(1-P) e^{\lambda T_{0}}$ is $J$-unitary. Clearly $e^{\lambda T_{0}}=C_{\lambda T_{0}} X_{\lambda T_{0}}$.

Proof. That $\left[P, e^{\lambda T_{0}}\right]=0$ is obvious, because $e^{\lambda T_{0}}$ does not mix terms of the $\tilde{l}^{2}$-sequences. Since Lemma 4.1 established that $e^{\lambda T_{0}}$ is symplectic, we only need to show that $\left[K, e^{\lambda T_{0}}\right] \neq 0$, and this will be entailed by the proof of $\left[K, C_{\lambda T_{0}}\right] \neq 0$ $=\left[K, X_{\lambda T_{0}}\right]$ which proves the second part. These follow by straightforward calculation, and we only do the first:

$$
\begin{aligned}
\left(K C_{\lambda T_{0}} f\right)_{n}^{(1)} & =\left(K e^{\lambda T_{0}} P f\right)_{n}^{(1)}=\delta_{n 0}\left(e^{\lambda T_{0}} f\right)_{0}^{(2)}=\delta_{n 0} f_{0}^{(2)} \quad \forall f \in S, \\
\left(C_{\lambda T_{0}} K f\right)_{n}^{(1)} & =\left(e^{\lambda T_{0}} K P f\right)_{n}^{(1)}=\delta_{n 0}\left[(K f)_{0}^{(1)}-2 \lambda \alpha^{\prime}(K f)_{0}^{(2)}\right] \\
& =\delta_{n 0}\left(f_{0}^{(2)}+2 \lambda \alpha^{\prime} f_{0}^{(1)}\right) \quad \forall f \in S,
\end{aligned}
$$

i.e. $\left[K, C_{\lambda T_{0}}\right] \neq 0$.

Recall the result of Mintchev mentioned in Sect. 3, viz for a J-unitary operator $V$, $\Gamma(V)$ is $\Gamma(J)$-unitary on $\mathscr{F}(S)$ and

$$
\Gamma(V) \Phi(f) \Gamma\left(V^{-1}\right) \chi=\Phi(V f) \chi \quad \forall \chi \in F_{0}^{R}, f \in D(V) .
$$

Corollary 4.4. (i) $\Gamma\left(e^{\lambda T_{0}}\right)$ is not a J-unitary implementer for the transformation $\Phi(f) \mapsto \Phi\left(e^{\lambda T_{0}} f\right)$.

(ii) $\Gamma\left(X_{\lambda T_{0}}\right) \Phi(f) \Gamma\left(X_{-\lambda T_{0}}\right) \chi=\Phi\left(X_{\lambda T_{0}} f\right) \chi \forall f \in S_{0}, \chi \in F_{00}$ and $\Gamma\left(X_{\lambda T_{0}}\right)$ constitutes a weakly continuous one parameter group of $J$-unitary operators.

Proof. Obvious. 
Theorem 4.5. (i) $p \cdot p$ is essentially self-adjoint, and hence by spectral theory we can construct $V_{\lambda}=\exp i \lambda \alpha^{\prime} \overline{p \cdot p}$, a unitary (and J-unitary) weak operator continuous one-parameter group.

(ii) The transformation $\Phi(f) \rightarrow \Phi\left(C_{\lambda T_{0}} f\right)$ is implementable by $V_{\lambda}$, and hence the transformation $\Phi(f) \rightarrow \Phi\left(e^{\lambda T_{0}} f\right)$ is implementable by $V_{\lambda} \Gamma\left(X_{\lambda T_{0}}\right)$, which is a weakly continuous one parameter group of $J$-unitaries.

Proof. (i) Since $p^{\mu}=\frac{i}{\sqrt{2}}\left(a^{+}(\delta(0, \mu))-a^{-}(\delta(0, \mu))\right)$ is a J-symmetric operator preserving the domain $F_{00}$, the same is true for $p \cdot p=-\left(p^{0}\right)^{2}+\left(p^{1}\right)^{2}+\cdots$ $+\left(p^{D}\right)^{2}$. So to show that $p \cdot p$ is in fact symmetric, it suffices to prove that $[\Gamma(J), p \cdot p] \chi=0 \forall \chi \in F_{00}$ and from the expansion of $p \cdot p$ in terms of $a^{ \pm}$, this will follow directly from Lemma 3.3. Now $p \cdot p$ is symmetric and of the kind of operator covered by Lemma 5.8, so it is essentially self-adjoint. Alternatively we can prove it by showing $F_{00}$ is contained in the set of analytic vectors of $p \cdot p$, then since $F_{00}$ is dense and invariant under $p \cdot p$, Nelson's analytic vector theorem implies that $p \cdot p$ is essentially self-adjoint. By spectral theory we define $e^{i \lambda \alpha^{\prime}} \overline{p \cdot p}$, where $\lambda, \alpha^{\prime} \in \mathbb{R}$ on $F_{00}$ which is then a weakly continuous one parameter group of unitaries (and J-unitaries).

(ii) We next prove that for all $\chi \in F_{00}, f \in S_{0}, \lambda \in \mathbb{R}$ :

$$
e^{i \lambda \alpha^{\prime} \overline{p \cdot p}} \Phi(f) e^{-i \lambda \alpha^{\prime} \overline{p \cdot p}} \chi=\Phi\left(C_{\lambda T_{0}}\right) \chi
$$

which will prove the theorem. Now $[p \cdot p, \Phi((I-P) f)] \chi=0$ for all $f \in S, \chi \in F_{0}$, so

$$
\left(\operatorname{Ad} e^{i \lambda \alpha^{\prime} \overline{p \cdot p}}\right)(\Phi(f)) \chi=\left[\left(\operatorname{Ad} e^{i \lambda \alpha^{\prime} \overline{p \cdot p}}\right)(\Phi(P f))+\Phi((I-P) f)\right] \chi
$$

Since $P f=f_{0}$, the zero mode, we have $P S \cong \mathbb{C}^{D+1}$ and

$$
\begin{aligned}
\mathscr{F}(S) & =\mathscr{F}(P S+(I-P) S)=\mathscr{F}(P S) \otimes \mathscr{F}((I-P) S) \\
& \cong L^{2}\left(\mathbb{C}^{D+1}\right) \otimes \mathscr{F}((I-P) S)
\end{aligned}
$$

and so for $\psi \in \mathscr{F}(P S) \cong L^{2}\left(\mathbb{C}^{D+1}\right), \phi \in \mathscr{F}((I-P) S)$,

$$
\left(\operatorname{Ad} e^{i \lambda \alpha^{\prime} \overline{p \cdot p}}\right)(\Phi(P f))(\psi \otimes \phi)=\left[\left(\operatorname{Ad} e^{i \lambda \alpha^{\prime} \overline{p \cdot p}}\right)(\Phi(P f)) \psi\right] \otimes \phi
$$

and the evaluation of the first factor is the same as evaluating the time evolution of a free particle in finite dimensional quantum mechanics:

$$
\begin{aligned}
e^{i \lambda \alpha^{\prime} \overline{p \cdot p}} \Phi(P f) e^{-i \lambda \alpha^{\prime} \overline{p \cdot p} \psi} & =e^{i \lambda \alpha^{\prime} \overline{p \cdot p}}\left(f_{0}^{(1)} \cdot p+f_{0}^{(2)} \cdot q\right) e^{-i \lambda \alpha^{\prime} \overline{p \cdot p} \psi} \\
& =\left(f_{0}^{(1)} \cdot p+f_{0}^{(2)} \cdot q+i \lambda \alpha^{\prime}\left[p \cdot p, f_{0}^{(2)} \cdot q\right]\right) \psi \\
& =\left(\left(f_{0}^{(1)}-2 \lambda \alpha^{\prime} f_{0}^{(2)}\right) \cdot p+f_{0}^{(2)} \cdot q\right) \psi \\
& =\Phi\left(P e^{\lambda T_{0}} f\right) \psi,
\end{aligned}
$$


where the second step followed from the Weyl relations and:

$$
\mathrm{s}-\lim _{t \rightarrow 0}\left(\operatorname{Ad} e^{i \lambda \overline{p \cdot p}}\right)\left(e^{i t q}-1\right) t^{-1} \psi=\left(\operatorname{Ad} e^{i \lambda \overline{p \cdot p}}\right)(i q) \psi .
$$

We could always assume that $\psi \in D(p) \cap D(q)$, since that followed from $\chi=\psi \otimes \phi \in F_{0}$. So:

$$
\begin{aligned}
\left(\operatorname{Ad} e^{i \lambda \alpha^{\prime} \overline{p \cdot p}}\right)(\Phi(f)) \chi & =\left(\Phi\left(P e^{\lambda T_{0}} f\right)+\Phi((I-P) f)\right) \chi \\
& =\Phi\left(C_{\lambda T_{0}} f\right) \chi \quad \forall \chi \in F_{00} .
\end{aligned}
$$

Clearly $\left[V_{\lambda}, \Gamma\left(X_{\lambda T_{0}}\right)\right]=0$.

So time evolutions are both integrable and J-unitarily implementable by a weakly continuous one parameter group. Application of spectral theory directly to $L_{0}$ would also have yielded the above J-unitary group.

We need to remark here about the apparent discrepancy with the finding in the geometric framework $[16,17,6]$ that the time evolutions commute with the complex structure $K$. This discrepancy is resolved by the observation that in the geometric framework the zero-mode (centre of mass variables) is omitted through the use of based loops, and in our framework the noncommuting aspect of $e^{\lambda T_{0}}$ with $K$ only shows up on the zero mode, i.e. $\left[K, C_{\lambda T_{0}}\right] \neq 0=\left[K, X_{\lambda T_{0}}\right]$. Since the gauge transformations as we shall see mix the zero-mode with other modes, we prefer to retain the zero mode in this formulation.

\section{Gauge Transformations}

In this section we intend to do for gauge transformations what we did for the time evolutions, i.e. find the infinitesimal gauge transformations and investigate integrability and implementability of these. In all fairness we need to point out to the reader forthcoming difficulties. We will find that the exponential series for the infinitesimal gauge transformations on $S$ have very unpleasant convergence properties (Prop. 5.3, 5.4). A suitable spectral theory to integrate up the infinitesimal gauge transformations, is also not forthcoming, one reason being that these are not complex linear operators with respect to the complex structure of the Fock-Krein representation. This forces us to make a decomposition of each gauge transformation into two components which are separately integrable, but do not commute. We prove implementability for these components and then use Trotter's product formula on the implementers to obtain the integrated gauge transformations on $S$ together with their implementers. The decompositions are introduced early in the analysis to establish notation, and any proof or derivation which is particularly algebraic or ugly, is banished to the appendix.

Since $\left\{L_{n}, n \neq 0\right\}$ are not J-hermitian, we take J-hermitian parts instead: $N_{n}:=L_{n}+L_{-n}$ and $M_{n}:=i\left(L_{n}-L_{-n}\right)$, so $N_{0}=2 L_{0}, N_{-n}=N_{n}, M_{-n}=-M_{n}$. We will recombine these in a suitable fashion at the point of selection of the physical vectors (or representations) according to the constraint conditions $\left(L_{n}-\alpha(0) \delta_{n, 0}\right)|\psi\rangle=0$ for all $n>0$. Then $\left\{i N_{n}, i M_{n} \mid n \in \mathbb{Z}\right\}$ will in fact generate a central extension of the Lie algebra $\operatorname{LDiff}^{+} S^{1}$, i.e. the Lie algebra of the real vector fields on the circle. Segal [11] integrated this central extension up to a central extension of the group $\operatorname{Diff}^{+} S^{1}$. In fact, the operators $N_{n}, M_{n}$ are the 
generators of infinitesimal gauge transformations of the string. Explicitly we calculate the commutation relations of this Lie algebra from the Virasoro relations to find $\forall \chi \in F_{00}$ and $n>0$ :

$$
\begin{aligned}
{\left[i L_{0}, i N_{n}\right] \chi } & =-n i M_{n} \chi, \quad\left[i L_{0}, i M_{n}\right] \chi=n i N_{n} \chi \\
{\left[i N_{n}, i N_{m}\right] \chi=} & (n-m) i M_{n+m} \chi+(n+m) i M_{n-m} \chi, \\
{\left[i M_{n}, i M_{m}\right] \chi=} & (m-n) i M_{n+m} \chi+(n+m) i M_{n-m} \chi \\
{\left[i M_{n}, i N_{m}\right] \chi=} & (m-n) i N_{n+m} \chi-(n+m) i N_{n-m} \chi \\
& -i \frac{D+1}{6} n\left(n^{2}-1\right)\left(\delta_{n+m, 0}+\delta_{n-m, 0}\right) \chi .
\end{aligned}
$$

The infinitesimal gauge transformations generated by $i N_{n}$ and $i M_{n}$ are calculated in the appendix with the results:

$$
\left(\operatorname{ad} i N_{n}\right)(\Phi(f)) \chi=\left[i N_{n}, \Phi(f)\right] \chi=\Phi\left(T_{n} f\right) \chi
$$

$\forall f \in S_{0}, \chi \in F_{00}$ and $n>0$ where:

$$
\begin{aligned}
& \left(T_{n} f\right)_{0}^{(1)}=2 \sqrt{2} \alpha^{\prime} n f_{n}^{(1)}, \quad\left(T_{n} f\right)_{0}^{(2)}=0, \\
& \left(T_{n} f\right)_{k}^{(1)}=(n-k) f_{|n-k|}^{(2)}-(n+k) f_{n+k}^{(2)}, \quad k>0, \\
& \left(T_{n} f\right)_{k}^{(2)}=|n-k| f_{|n-k|}^{(1)}+(n+k) f_{n+k}^{(1)}-\sqrt{2} f_{0}^{(2)} \delta_{k}^{n}, \quad k>0
\end{aligned}
$$

and $\forall f \in S_{0}, \chi \in F_{00}, n>0$ we have:

$$
\left(\operatorname{ad} i M_{n}\right)(\Phi(f)) \chi=\Phi\left(R_{n} f\right) \chi
$$

where

$$
\begin{aligned}
& \left(R_{n} f\right)_{0}^{(1)}=n 2 \sqrt{2} \alpha^{\prime} f_{n}^{(2)}, \quad\left(R_{n} f\right)_{0}^{(2)}=0, \\
& \left(R_{n} f\right)_{k}^{(1)}=(n+k) f_{n+k}^{(1)}-|n-k| f_{|n-k|}^{(1)}+\sqrt{2} f_{0}^{(2)} \delta_{k}^{n}, \quad k>0, \\
& \left(R_{n} f\right)_{k}^{(2)}=(n+k) f_{n+k}^{(2)}+(n-k) f_{|n-k|}^{(2)}, \quad k>0 .
\end{aligned}
$$

Then $T_{n}$ and $R_{n}$ preserve $S_{0}$ and are infinitesimally symplectic (cf. (A.5, 6)):

$$
B\left(T_{n} f, h\right)+B\left(f, T_{n} h\right)=0=B\left(R_{n} f, h\right)+B\left(f, R_{n} h\right) \quad \forall f, h \in S_{0}, n \geqq 1 .
$$

Since $N_{-n}=N_{n}$ and $M_{-n}=-M_{n}$, we also set $T_{-n}=T_{n}$ and $R_{-n}=-R_{n}$ for all $n \geqq 1$, and $R_{0}=0$. Note that $T_{n}$ and $R_{n}$ are unbounded operators, hence they cannot be in the Lie algebra of $\operatorname{Sp}(\infty)$, so one cannot use Milnor's theorem (cf. [22]) for integrating Lie algebra homomorphisms up to Lie group homomorphisms of the corresponding (infinite dimensional) groups.

Since $T_{n}$ and $R_{n}$ are also non-complex linear, i.e. $\left[T_{n}, K\right] \neq 0 \neq\left[R_{n}, K\right]$ as an easy calculation shows, care must be taken in the analysis of these. This observation corresponds to the similar finding of Bowick and Rajeev $[16,17]$ regarding the non-gauge invariance of their complex structure. As for the domains of the operators $T_{n}$ and $R_{n}$, we will take it to be $S_{0}=D\left(T_{n}\right)=D\left(R_{n}\right)$ throughout.

Next we examine the structure of $T_{n}$ and $R_{n}$ in order to obtain some useful decompositions into more manageable parts before looking at integrability. Since 
$T_{n}, R_{n}$ act as the identity on the $M^{D+1}$ part of $S_{0}=\mathbb{R}^{2} \otimes M^{D+1} \otimes l_{0}$, we can express these operators as $2 \times 2$ matrices of which the entries are operators on $l_{0}$, and these entries can then be expressed as infinite matrices. In what follows space-time indices will be omitted. First, write $T_{n}$ as a $2 \times 2$ matrix: $T_{n}=\left(\begin{array}{ll}t_{n} & s_{n} \\ p_{n} & r_{n}\end{array}\right)$, where $\left(t_{n} f\right)_{k}=\delta_{k, 0} 2 \sqrt{2} \alpha^{\prime} f_{n}$ and $\left(r_{n} f\right)_{k}=-\delta_{k, n} \sqrt{2} f_{0} \forall f \in l_{0}$, and for all $f \in l_{0}$, $n \geqq 1, k>0$ :

$$
\left(s_{n} f\right)_{k}=(n-k) f_{|n-k|}-(n+k) f_{n+k}, \quad\left(p_{n} f\right)_{k}=|n-k| f_{|n-k|}+(n+k) f_{n+k} .
$$

The structure of the last two operators become clearer when these are expressed as infinite matrices:

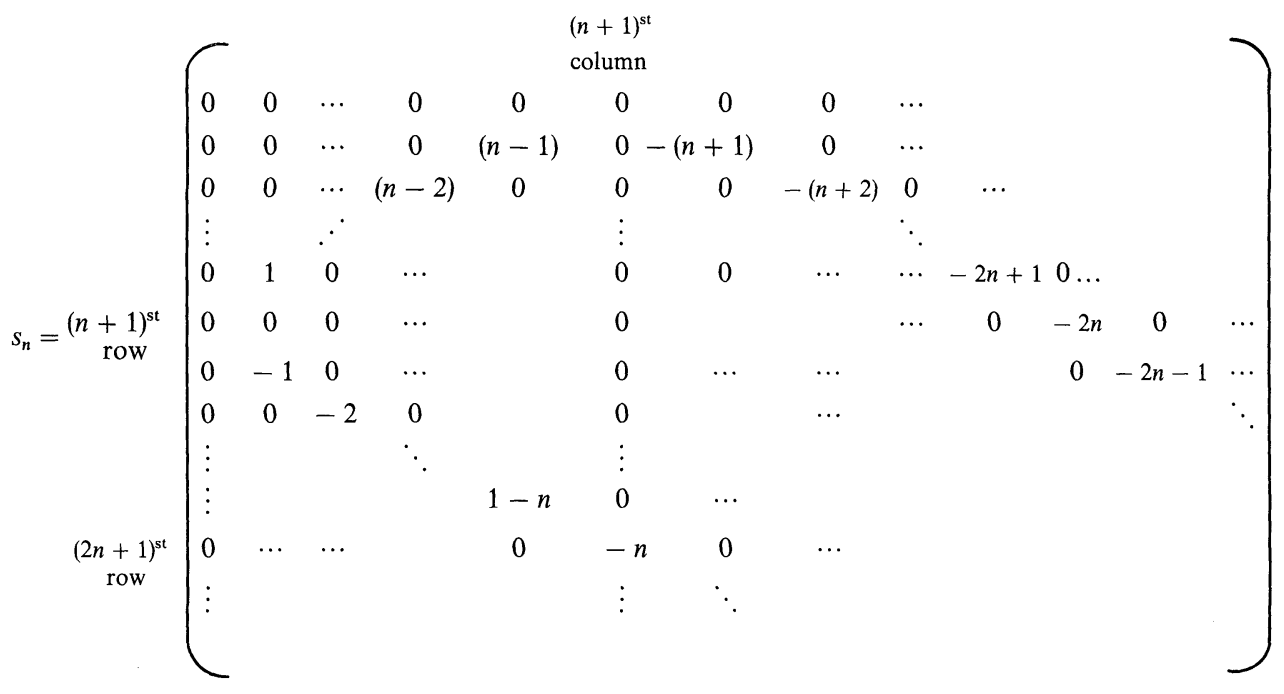

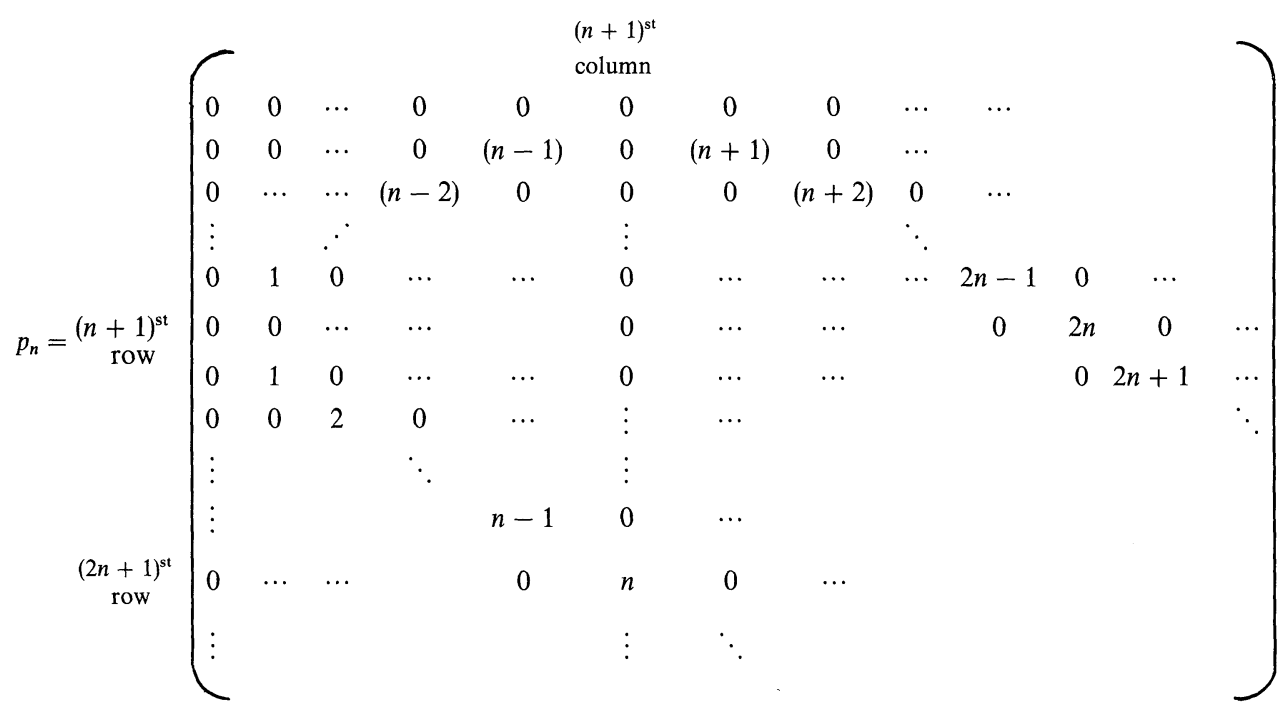


Likewise for $R_{n}$ we write $==\left(\begin{array}{cc}u_{n} & q_{n} \\ 0 & v_{n}\end{array}\right)$ where for all $f \in l_{0}$ :

$$
\left(q_{n} f\right)_{k}=2 \sqrt{2} n \alpha^{\prime} f_{n} \delta_{k, 0}+\sqrt{2} f_{0} \delta_{k, n},
$$

i.e. in matrix format

$$
(n+1)^{\mathrm{st}}
$$

column

$$
q_{n}=\underset{(n+1)^{\text {st }}}{\operatorname{row}}\left(\begin{array}{cccccccc}
0 & 0 & \cdots & 0 & 2 \sqrt{2} n \alpha^{\prime} & 0 & \cdots \\
0 & 0 & \cdots & 0 & 0 & 0 & \cdots \\
\vdots & \vdots & & \vdots & \vdots & \vdots & \\
0 & 0 & \cdots & \vdots & \vdots & \vdots & \\
\sqrt{2} & 0 & \cdots & 0 & 0 & 0 & \cdots \\
0 & 0 & \cdots & \vdots & \vdots & \vdots & \\
\vdots & \vdots & & \vdots & \vdots & &
\end{array}\right)
$$

and

$$
\begin{aligned}
& \left(u_{n} f\right)_{k}=(n+k) f_{n+k}-|n-k| f_{|n-k|}, \\
& \left(v_{n} f\right)_{k}=(n+k) f_{n+k}+(n-k) f_{|n-k|}
\end{aligned}
$$

for all $f \in l_{0}$, i.e.

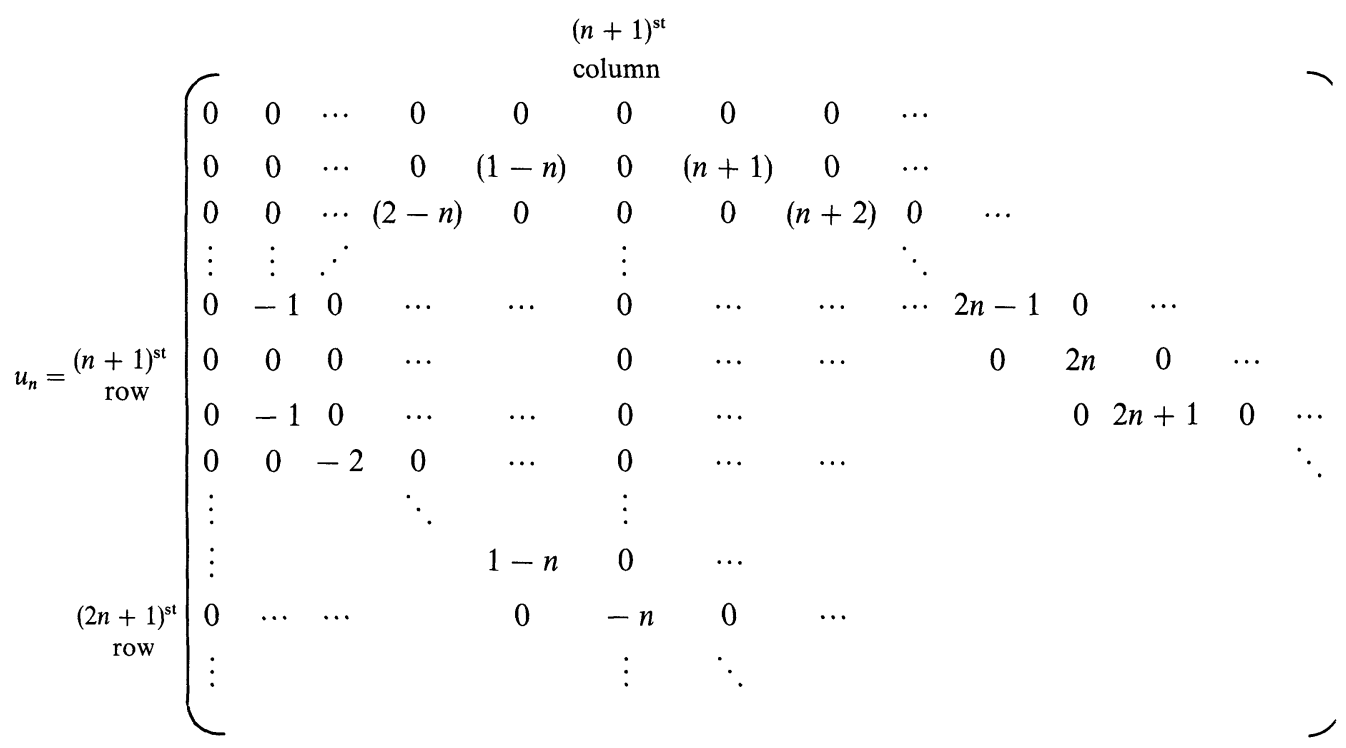




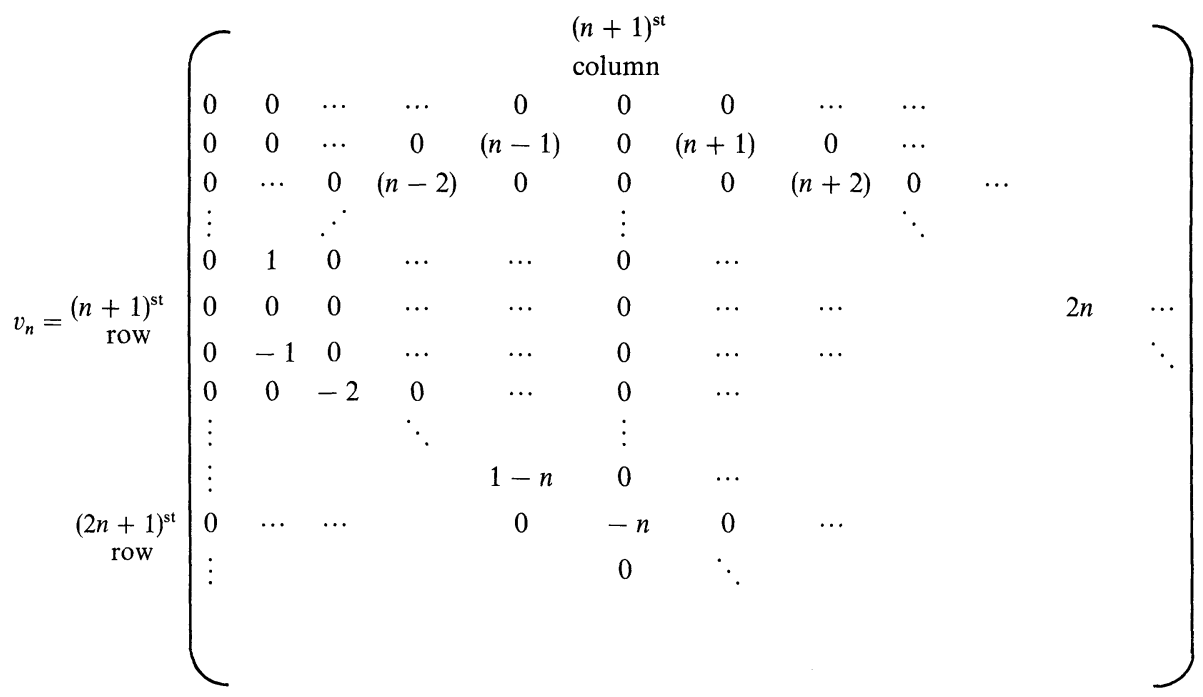

Now for a complex linear operator $A$ we need $[A, K]=0$. The general form of a $2 \times 2$ matrix which commutes with $K=\left(\begin{array}{rr}0 & 1 \\ -1 & 0\end{array}\right)$ is $\left(\begin{array}{rr}a & b \\ -b & a\end{array}\right)$. Observe that the infinite tails of $T_{n}$ and $R_{n}$ (i.e. everything outside the first $(n+1) \times(n+1)$ block) will be of exactly this form. This suggests a useful decomposition of $T_{n}$ and $R_{n}$. Let $P_{n}$ be the projection of $S$ on to the subspace spanned by the first $n$ excitation modes (i.e. from 0 up to $(n-1))$. Then for an operator $A$ on $S, P_{n} A P_{n}$ will be the first $n \times n$ block in the matrix notations above, and hence is a bounded operator of (complex) rank less than $n$. So we form the decompositions:

$$
\begin{aligned}
& T_{n}=\dot{T}_{n}+\tilde{T}_{n} \quad \text { with } \quad \dot{T}_{n}:=P_{n} T_{n} P_{n} \quad \text { and } \quad \tilde{T}_{n}:=T_{n}-\dot{T}_{n}, \\
& R_{n}=\dot{R}_{n}+\tilde{R}_{n} \quad \text { with } \quad \dot{R}_{n}:=P_{n} R_{n} P_{n} \quad \text { and } \quad \tilde{R}_{n}:=R_{n}-\dot{R}_{n},
\end{aligned}
$$

then $\left[K, \tilde{T}_{n}\right]=0=\left[K, \tilde{R}_{n}\right]$ and $\dot{T}_{n}, \dot{R}_{n}$ are finite rank (less than $n$ ) operators. It is also not hard to see that $\left[\dot{T}_{n}, \tilde{T}_{n}\right] \neq 0 \neq\left[\dot{R}_{n}, \tilde{R}_{n}\right]$ though these commutators will of course be finite rank operators. Explicitly we have: $\dot{T}_{n}=\left(\begin{array}{cc}t_{n} & \dot{s}_{n} \\ \dot{s}_{n} & r_{n}\end{array}\right)$ where:

$$
\dot{s}_{n}=P_{n} s_{n} P_{n}=P_{n} p_{n} P_{n}=\left(\begin{array}{cccccccc}
0 & \cdots & \cdots & & 0 & 0 & 0 & \ldots \\
\vdots & & & & 0 & (n-1) & 0 & \ldots \\
\vdots & & & 0 & (n-2) & 0 & 0 & \ldots \\
\vdots & & & \therefore & & & \vdots & \\
0 & 1 & 0 & \cdots & & & \vdots & \\
0 & \cdots & \cdots & & & & 0 & \ldots \\
\vdots & & & & & & \vdots &
\end{array}\right)
$$


Then by inspection of the matrices we see $s_{n}-\dot{s}_{n}=-\left(p_{n}-\dot{s}_{n}\right)=: \tilde{s}_{n}$, so $\tilde{T}_{n}=\left(\begin{array}{cc}0 & \tilde{s}_{n} \\ -\tilde{s}_{n} & 0\end{array}\right)$, hence $\left[K, \tilde{T}_{n}\right]=0 \neq\left[K, \dot{T}_{n}\right]$ and by evaluation of $\dot{s}_{n} \tilde{s}_{n}+\tilde{s}_{n} \dot{s}_{n}$ we see that

$$
\left[\dot{T}_{n}, \tilde{T}_{n}\right]=\left(\begin{array}{cc}
-\dot{s}_{n} \tilde{s}_{n}-\tilde{s}_{n} \dot{s}_{n} & t_{n} \tilde{s}_{n}-\tilde{s}_{n} r_{n} \\
\tilde{s}_{n} t_{n}-r_{n} \tilde{s}_{n} & \tilde{s}_{n} \dot{s}_{n}+\dot{s}_{n} \tilde{s}_{n}
\end{array}\right) \neq 0
$$

Likewise

$$
\dot{R}_{n}=\left(\begin{array}{cc}
-\dot{s}_{n} & q_{n} \\
0 & \dot{s}_{n}
\end{array}\right) \text { and } \tilde{R}_{n}=\left(\begin{array}{cc}
\tilde{u}_{n} & 0 \\
0 & \tilde{u}_{n}
\end{array}\right)
$$

where $\tilde{u}_{n}:=u_{n}+\dot{s}_{n}=v_{n}-\dot{s}_{n}$, hence $\left[K, \tilde{R}_{n}\right]=0 \neq\left[K, \dot{R}_{n}\right]$. So now we have the decompositions $T_{n}=\dot{T}_{n}+\tilde{T}_{n}, R_{n}=\dot{R}_{n}+\tilde{R}_{n}$, where $\dot{T}_{n}$ and $\dot{R}_{n}$ are (real) rank $2(n+1)$ operators and $\tilde{T}_{n}, \tilde{R}_{n}$ commute with $K$. The methods of Araki [7], developed to deal with non-complex linear symplectic transformations, did not provide any stronger analytic tools than those already used here.

Next we need to examine the closability and adjoints of $\tilde{T}_{n}, \tilde{R}_{n}$. In the examination we exploit the fact that $T_{n}, R_{n}$ act as the identity on the $M^{D+1}$ part of $S=\mathbb{R}^{2} \otimes M^{D+1} \otimes \tilde{l}^{2}$ which contributes the indefinite part to the inner product of $S$, and hence $T_{n}, R_{n}$ can be considered as (unbounded) Hilbert space operators on the Hilbert space $\mathbb{R}^{2} \otimes \tilde{l}^{2}$, with dense domains containing $\mathbb{R}^{2} \otimes l_{0}$. Hence ordinary Hilbert space operator theory and spectral theory is applicable to $R_{n}, T_{n}$ and their various decompositions.

Lemma 5.1. $\tilde{T}_{n}, \tilde{R}_{n}$, are closable complex linear operators which are essentially antiselfadjoint. Since $\left[J, \tilde{T}_{n}\right]=0=\left[J, \tilde{R}_{n}\right]$, they are also essentially anti-Jselfadjoint.

Proof. Let $A=\tilde{T}_{n}$ or $\tilde{R}_{n}$. By definition $D\left(A^{+}\right):=\{f \in S \mid \exists h \in S$ s.t. $\left.\langle h, k\rangle_{K}=\langle f, A k\rangle_{K} \forall k \in D(A)\right\}=D\left(A^{*}\right)$ since $[A, J]=0$ and $J$ preserves $S_{0}$. Decomposing $\langle h, k\rangle_{\mathrm{K}}=\langle f, A k\rangle_{\mathrm{K}}$, we get

$$
\begin{aligned}
\langle h, k\rangle_{K} & =B(h, K k)+i B(h, k) \\
& =\langle f, A k\rangle_{K}=B(f, K A k)+i B(f, A k),
\end{aligned}
$$

i.e.

$$
B(h, K k)=B(f, K A k) \quad \text { and } \quad B(h, k)=B(f, A k) .
$$

That is $f \in D\left(A^{*}\right)$ iff there exists an $h \in S$ such that $B(h, k)=B(f, A k) \forall k \in S_{0}$ (since $[A, K]=0$ and $K$ preserves $\left.S_{0}\right)$. Recall $\tilde{T}_{n}=\left(\begin{array}{cc}0 & \tilde{s}_{n} \\ -\tilde{s}_{n} & 0\end{array}\right)$ with $\left(\tilde{s}_{n} f\right)_{0}=0$ and $\left(\tilde{s}_{n} f\right)_{k}=-(n+k) f_{n+k}-(k-n) \theta(k-n) f_{k-n} \forall f \in l_{0}, k>0$, where $\theta(k):=\{0$ if $k \leqq 0 ; 1$ if $k>1\}$. Also $\tilde{R}_{n}=\left(\begin{array}{cc}\tilde{u}_{n} & 0 \\ 0 & \tilde{u}_{n}\end{array}\right)$ with $\left(\tilde{u}_{n} f\right)_{0}=0, \quad\left(\tilde{u}_{n} f\right)_{k}=(n+k) f_{n+k}$ $-(k-n) \theta(k-n) f_{k-n} \forall f \in l_{0}, k>0$. So $f \in D\left(\tilde{T}_{n}^{*}\right)$ iff for all $k \in S_{0}$ (suppressing 
space-time indices):

$$
\begin{aligned}
B\left(f, \tilde{T}_{n} k\right)= & 2 \alpha^{\prime} \sum_{j=1}^{\infty} j\left(f_{j}^{(1)}\left(\tilde{T}_{n} k\right)_{j}^{(2)}-f_{j}^{(2)}\left(\tilde{T}_{n} k\right)_{j}^{(1)}\right) \\
= & 2 \alpha^{\prime} \sum_{j=1}^{\infty} j(n+j)\left(f_{j}^{(1)} k_{n+j}^{(1)}+f_{j}^{(2)} k_{n+j}^{(2)}\right) \\
& +2 \alpha^{\prime} \sum_{j=n+1}^{\infty} j(j-n)\left(f_{j}^{(1)} k_{j-n}^{(1)}+f_{j}^{(2)} k_{j-n}^{(2)}\right) \\
= & 2 \alpha^{\prime} \sum_{j=n+1}^{\infty}(j-n) j\left(f_{j-n}^{(1)} k_{j}^{(1)}+f_{j-n}^{(2)} k_{j}^{(2)}\right) \\
& +2 \alpha^{\prime} \sum_{j=1}^{\infty}(j+n) j\left(f_{n+j}^{(1)} k_{j}^{(1)}+f_{n+j}^{(2)} k_{j}^{(2)}\right) \\
= & B(h, k)=h_{0}^{(1)} k_{0}^{(2)}-h_{0}^{(2)} k_{0}^{(1)}+2 \alpha^{\prime} \sum_{j=1}^{\infty} j\left(h_{j}^{(1)} k_{j}^{(2)}-h_{j}^{(2)} k_{j}^{(1)}\right) .
\end{aligned}
$$

So since this is true for all $k \in S_{0}$, we obtain

$$
\begin{aligned}
& h_{0}^{(i)}=:\left(\tilde{T}_{n}^{+} f\right)_{0}^{(i)}=0, \quad i=1,2, \quad \text { and } \\
& h_{k}^{(1)}=:\left(\tilde{T}_{n}^{+} f\right)_{k}^{(1)}=(n+k) f_{n+k}^{(2)}+(k-n) \theta(k-n) f_{k-n}^{(2)}=-\left(\tilde{s}_{n} f^{(2)}\right)_{k}, \\
& h_{k}^{(2)}=:\left(\tilde{T}_{n}^{+} f\right)_{k}^{(2)}=-(n+k)\left(f_{n+k}^{(1)}-(k-n) \theta(k-n) f_{k-n}^{(1)}=\left(\tilde{s}_{n} f^{(1)}\right)_{k},\right.
\end{aligned}
$$

where $k \geq 0$. Thus $f \in D\left(\tilde{T}_{n}^{*}\right)$ if these expressions define an element of $S$. Clearly $S_{0} \subset D\left(\tilde{T}_{n}^{*}\right)$ and so since this is dense in $S, \tilde{T}_{n}$ is closable. Moreover we have also shown that $\tilde{T}_{n}^{+} \supset-\tilde{T}_{n}=\left(\begin{array}{cc}0 & -\tilde{s}_{n} \\ \tilde{s}_{n} & 0\end{array}\right)$, i.e. $K \tilde{T}_{n}=\left(\begin{array}{cc}\tilde{s}_{n} & 0 \\ 0 & \tilde{s}_{n}\end{array}\right)$ is symmetric, using $\left[J, \tilde{T}_{n}\right]=0$.

To prove that all $h \in S_{0}=D\left(\tilde{T}_{n}\right)=C^{\infty}\left(\tilde{T}_{n}\right)$ are analytic vectors for $K \tilde{T}_{n}$, it suffices to show that $\sum_{k=0}^{\infty} \frac{\lambda^{k}}{k !}\left\|\left(\tilde{s}_{n}\right)^{k} \varepsilon_{m}\right\|<\infty$ for some $\lambda>0$ and for all $m>0$, where $\varepsilon_{m}$ is the unit vector for the $m^{\text {th }}$ mode in $l_{0}$. Now using exactly the same argument as in the proof of Lemma A.8, we find

$$
\left\|\left(\tilde{s}_{n}\right)^{k} \varepsilon_{m}\right\| \leqq \sqrt{\alpha^{\prime}} 2^{k+1} \prod_{l=0}^{k}(m+\ln )
$$

(since $\tilde{s}_{n} \varepsilon_{m}$ is $S_{n} \varepsilon_{m}$ with some terms omitted). So

$$
\sum_{k=0}^{\infty} \frac{\lambda^{k}}{k !}\left\|\left(\tilde{s}_{n}\right)^{k} \varepsilon_{m}\right\| \leqq 2 \sqrt{\alpha^{\prime}} \sum_{k=0}^{\infty} \frac{(2 \lambda)^{k}}{k !} \prod_{l=0}^{k}(m+\ln )=: 2 \sqrt{\alpha^{\prime}} \sum_{k=0}^{\infty} c_{k}
$$

and on application of the ratio test:

$$
\left|\frac{c_{k+1}}{c_{k}}\right|=\frac{2 \lambda}{k+1}(m+(k+1) n) \underset{\infty}{\stackrel{k}{\longrightarrow}} 2 \lambda n .
$$

Hence the series converges for $\lambda<1 / 2 n$, and so $S_{0}$ is a dense invariant set of analytic vectors for $K \tilde{T}_{n}$, hence by Nelson's theorem $K \tilde{T}_{n}$ is essentially selfadjoint, i.e. $\tilde{T}_{n}$ is essentially anti-selfadjoint. $\tilde{R}_{n}$ follows similarly. 
Observe that in the proof above we showed that for all $f, k \in S_{0}, B\left(f, \tilde{T}_{n} k\right)=$ $B\left(\tilde{T}_{n}^{+} f, k\right)=-B\left(\tilde{T}_{n} f, k\right)$ and the same for $\tilde{R}_{n}$, i.e. $\tilde{T}_{n}$ and $\tilde{R}_{n}$ are infinitesimally symplectic. Since $T_{n}$ and $R_{n}$ are also infinitesimally symplectic (cf. Appendix), this implies the same property for the remaining part of the decompositions $\dot{T}_{n}$ and $\dot{R}_{n}$. However $\dot{T}_{n}$ and $\dot{R}_{n}$ are not antihermitian since $\left[K, \dot{T}_{n}\right] \neq 0 \neq\left[K, \dot{R}_{n}\right]$, and on the complex Hilbert space $\mathbb{R}^{2} \otimes \widetilde{l}^{2}$, are real linear but not complex linear. This means that we cannot apply the classical Weyl theorem on the spectra of compact perturbations. Moreover, exponentiation of $T_{n}, R_{n}, \dot{T}_{n}, \dot{R}_{n}$, will not produce unitary operators, so that these transformations are not implementable via the map $\Gamma$ (cf. Sect. 4).

Corollary 5.2. $T_{n}$ and $R_{n}$ are closable operators.

Proof. $\tilde{T}_{n}$ is densely defined and closable, $\dot{T}_{n}$ is bounded hence continuous and a closed operator. So using $D\left(\tilde{T}_{n}\right) \cap D\left(\dot{T}_{n}\right)=D\left(\tilde{T}_{n}\right), T_{n}$ is closable. $R_{n}$ follows similarly.

Now in order to go to a $\mathrm{C}^{*}$-algebra framework, we need to exponentiate $T_{n}$ and $R_{n}$ into symplectic operators on $S$. Since these are not complex linear, the Hilbertspace spectral theory of $S$ will not work directly, and if we only consider the real linear theory in order to construct a complex Hilbert theory on $S$ as a real Hilbert space, the spectral theory on the new space will also not help because these operators are not normal. So next we consider the convergence properties of the exponential series with relation to the Hilbert-space topology. (Similar convergence properties are obtained with relation to the weak star topology of $S_{0}$ on $S$.)

Proposition 5.3. The exponential series $\sum_{k=0}^{\infty} \frac{\lambda^{k} T_{n}^{k}}{k !} f, n>0$ converges absolutely in the Hilbert norm of $S$ for all $f \in S_{0}$ and for all $\lambda \in\left(-\frac{1}{2 n}, \frac{1}{2 n}\right)$. This series diverges in the Hilbert norm topology for all nonzero $f \in S_{0}$ and $\lambda \in \mathbb{R} \backslash\left(-\frac{1}{n}, \frac{1}{n}\right)$. In the region of convergence, denote the limit by $\exp \left(\lambda T_{n}\right) f, n>0$.

The proof of this is lengthy and has been placed in the appendix, Prop. A.6.

Proposition 5.4. (i) $\exp \lambda T_{n}$ is an unbounded operator on $S$ for nonzero $|\lambda|<1 / 2 n$ with $S_{0}$ in its domain.

(ii) Given $\lambda, \mu \in \mathbb{R}$ such that $|\lambda|,|\mu|<1 / 2 n$ and $|\lambda+\mu|<1 / 2 n$, then the power series of $\exp \lambda T_{n}$ converges on $\left(\exp \mu T_{n}\right)\left(S_{0}\right)$, and

$$
\left(\exp \lambda T_{n}\right)\left(\left(\exp \mu T_{n}\right) f\right)=\left(\exp (\lambda+\mu) T_{n}\right) f \quad \forall f \in S_{0},
$$

where we maintain the notation $\exp \lambda T_{n}$ on this larger domain.

Proof. (i) We only need to show that $\exp \lambda T_{n}$ is unbounded. Given a converging sequence of the form $\left\{f_{N}:=\sum_{k=1}^{N} \gamma_{k} \delta_{k}^{(1)} \cdot\right\}_{N \in \mathbb{N}}$ in $S_{0}$, converging to an $f \in S$ (hence we must have $\left.\sum_{k=1}^{\infty} \gamma_{k}^{2} k<\infty\right)$, we consider:

$$
\mathrm{s}-\lim \left(\exp \lambda T_{n}\right) f_{N}=\mathrm{s}-\lim \mathrm{s}-\lim \sum_{N \rightarrow \infty}^{N} \gamma_{k \rightarrow \infty} \sum_{k=1}^{M} \frac{\left(\lambda T_{n}\right)^{m}}{m !} \delta_{k}^{(1)} .
$$


Now

$$
\begin{aligned}
\left\|\gamma_{k} \frac{\left(\lambda T_{n}\right)^{m}}{m !} \delta_{k}^{(1)}\right\| & \geqq\left|\gamma_{k}\right| \sqrt{2 \alpha^{\prime}(k+m n)} \frac{|\lambda|^{m}}{m !} \prod_{l=0}^{m-1}(k+l n) \\
& \geqq\left|\gamma_{k}\right| \sqrt{2 \alpha^{\prime} k} \frac{(k|\lambda|)^{m}}{m !},
\end{aligned}
$$

where use was made of A.9. So:

$$
\sum_{m=0}^{\infty}\left|\gamma_{k}\right| \sqrt{2 \alpha^{\prime} k}(k|\lambda|)^{m} / m !=\left|\gamma_{k}\right| \sqrt{2 \alpha^{\prime} k} e^{|\lambda| k} .
$$

The series $\sum_{k=1}^{\infty}\left|\gamma_{k}\right| \sqrt{2 \alpha^{\prime} k} e^{|\lambda| k}$ will certainly diverge if $\left|\gamma_{k}\right| \sqrt{k} e^{\lambda k}$ does not tend to zero as $k$ goes to infinity, and this is quite easy to arrange by choosing e.g. $\gamma_{k}=k^{-2}$. Then $f_{N} \underset{\infty}{\stackrel{N}{\longrightarrow}} f=\sum_{k=1}^{\infty} k^{-2} \delta_{k}^{(1)} \in S$, but s- $\lim _{N \rightarrow \infty}\left(\exp \lambda T_{n}\right) f_{N}$ does not exist, i.e. $\exp \lambda T_{n}$ is unbounded.

(ii) Let $\mu, \lambda$ be as given. Since $\sum_{k=0}^{\infty} \frac{\left[(\lambda+\mu) T_{n}\right]^{k}}{k !} f$ converges absolutely for all $f \in S_{0}$, we can rearrange its terms without affecting convergence:

$$
\begin{aligned}
\left(\exp (\lambda+\mu) T_{n}\right) f & =\sum_{k=0}^{\infty} \frac{\left[(\lambda+\mu) T_{n}\right]^{k}}{k !} f=\sum_{k=0}^{\infty} \frac{1}{k !} \sum_{l=0}^{k} \frac{k !}{l !(k-l) !} \lambda^{l} \mu^{k-l}\left(T_{n}\right)^{k} f \\
& =\sum_{k=0}^{\infty} \sum_{l=0}^{\infty} \frac{\lambda^{k} \mu^{l}}{k ! l !}\left(T_{n}\right)^{k+l} f \\
& =\operatorname{s-lim}\left(\operatorname{s}_{M \rightarrow \infty} \sum_{M \rightarrow \infty}^{N} \frac{\left(\lambda T_{n}\right)^{k}}{k !} \sum_{l=0}^{M} \frac{\left(\mu T_{n}\right)^{l}}{l !} f\right) \\
& =\mathrm{s}-\lim _{N \rightarrow \infty} \sum_{k=0}^{N} \frac{\left(\lambda T_{n}\right)^{k}}{\mathrm{k} !}\left(\exp \mu T_{n}\right) f,
\end{aligned}
$$

where the order of the limits does not matter due to the absolute convergence. It is clear that the last limit exists, i.e. the power series of $\exp \lambda T_{n}$ converges on the region $\left(\exp \mu T_{n}\right)\left(S_{0}\right)$.

To construct a $C^{*}$-algebra expression of the string, we need a symplectic space containing $\left\{S_{0}, B\right\}$ to model the excitation modes, together with all relevant transformations written as symplectic transformations on this space. Now by 5.4(i), we know that $\exp \lambda T_{n}$ cannot be extended by continuity to $S$. If there is some subspace of $S$ containing $S_{0}$ which is preserved by all $\exp \lambda T_{n}, 0<|\lambda|<1 / 2 n$, then it is possible to compose enough $\exp \lambda T_{n}$ 's to obtain a convergent exponential series on $S_{0}$ (using the absolute convergence of the factors) which will violate the divergence part of 5.3. Hence we are forced to conclude that $T_{n}$ for $n \neq 0$ does not integrate up to a symplectic transformation group in a useful manner via an exponential series. Moreover, since $T_{n}$ is neither complex linear, nor normal as a real linear operator, we cannot use spectral theory to define $\exp \lambda T_{n}$.

Next we will find the decomposition of the infinitesimal gauge generators ${\underset{\sim}{n}}_{n}$ and $M_{n}$ corresponding to the decompositions $T_{n}=\dot{T}_{n}+\tilde{T}_{n}, R_{n}=\dot{R}_{n}+\tilde{R}_{n}$. 
Consider for $n \geqq 1$ the decomposition of $L_{n}$ :

$$
L_{n}=\dot{L}_{n}+\tilde{L}_{n}=\frac{1}{2}\left(\dot{N}_{n}+\tilde{N}_{n}-i \dot{M}_{n}-i \tilde{M}_{n}\right)
$$

where

$$
\begin{gathered}
\dot{L}_{n}:=\frac{1}{4 \alpha^{\prime}} \sum_{k=1}^{n-1} \alpha_{k} \cdot \alpha_{n-k}+\alpha_{n} \cdot p, \quad \tilde{L}_{n}:=\frac{1}{2 \alpha^{\prime}} \sum_{k=1}^{\infty} \alpha_{-k} \cdot \alpha_{n+k}, \\
\left(\dot{L}_{-n}\right)^{+}=\dot{L}_{n}, \quad\left(\tilde{L}_{-n}\right)^{+}=\tilde{L}_{n}, \\
\dot{N}_{n}=\dot{L}_{n}+\dot{L}_{-n}, \quad \tilde{N}_{n}=\tilde{L}_{n}+\tilde{L}_{-n}, \quad N_{n}=\dot{N}_{n}+\tilde{N}_{n}, \\
\dot{M}_{n}=i\left(\dot{L}_{n}-\dot{L}_{-n}\right), \quad \tilde{M}_{n}=i\left(\tilde{L}_{n}-\tilde{L}_{-n}\right), \quad M_{n}=\dot{M}_{n}+\tilde{M}_{n} .
\end{gathered}
$$

\section{Lemma 5.5.}

$$
\begin{aligned}
{\left[i \dot{N}_{n}, \Phi(f)\right] \chi } & =\Phi\left(\dot{T}_{n} f\right) \chi, \\
{\left[i \dot{M}_{n}, \Phi(f)\right] \chi } & =\Phi\left(\dot{R}_{n} f\right) \chi \quad \forall f \in S_{0}, \quad \chi \in F_{00}, \quad n \geqq 1 .
\end{aligned}
$$

Proof. Appendix A.5.

Lemma 5.5 immediately implies that

$$
\begin{aligned}
& {\left[i \tilde{N}_{n}, \Phi(f)\right] \chi=\Phi\left(\tilde{T}_{n} f\right) \chi,} \\
& {\left[i \tilde{M}_{n}, \Phi(f)\right] \chi=\Phi\left(\tilde{R}_{n} f\right) \chi \quad \forall f \in S_{0}, \quad \chi \in F_{00}, \quad n \geqq 1 .}
\end{aligned}
$$

Hence the decomposition $L_{n}=\dot{L}_{n}+\tilde{L}_{n}$ forces exactly the decompositions $T_{n}=\dot{T}_{n}+\tilde{T}_{n}$ and $R_{n}=\dot{R}_{n}+\widetilde{T}_{n}$ constructed before, and we already know (cf. 5.1$)$ that $\tilde{T}_{n}, \tilde{R}_{n}$ are integrable via spectral theory, and $\dot{T}_{n}, \dot{R}_{n}$ are integrable because they are finite rank operators, and so their exponential series always converge absolutely. So by Stone's theorem $\left\{\exp \lambda x \mid x=\widetilde{T}_{n}, \tilde{R}_{n} ; \lambda \in \mathbb{R}, n \geqq 1\right\}$ are one-parameter unitary groups (hence J-unitary groups since $\left[J, \tilde{T}_{n}\right]=0=\left[J, \tilde{R}_{n}\right]$ ) and hence since the symplectic form $B$ is the imaginary part of the inner product, this implies that these families are also symplectic operators on $S$.

On the other hand, the families $\exp \lambda \dot{T}_{n}, \exp \lambda \dot{R}_{n}$ on $S$ are not unitary nor J-unitary since $\left[\dot{T}_{n}, K\right] \neq 0 \neq\left[\dot{R}_{n}, K\right]$.

Lemma 5.6. $\exp \lambda \dot{T}_{n}$ and $\exp \lambda \dot{R}_{n}$ are symplectic real linear operators for all $\lambda \in \mathbb{R}$, and $n \in \mathbb{Z}^{*}$.

Proof. Since $\dot{T}_{n}$ and $\dot{R}_{n}$ are finite rank matrices, the series $\exp \lambda \dot{T}_{n} f$, etc. will converge absolutely for all $f \in S$. Since the symplectic form is jointly continuous with respect to the Hilbert norm topology, the result follows by rearrangement of the series, i.e.

$$
\begin{aligned}
B\left(\exp \lambda \dot{T}_{n} f, \exp \lambda \dot{T}_{n} h\right) & =\lim _{K \rightarrow \infty} \lim _{N \rightarrow \infty} \sum_{k=0}^{K} \sum_{r=0}^{N} B\left(\left(\lambda \dot{T}_{n}\right)^{k} f,\left(\lambda \dot{T}_{n}\right)^{r} h\right) / k ! r ! \\
& =\lim _{K \rightarrow \infty} \lim _{N \rightarrow \infty} \sum_{k=0}^{K} \sum_{r=0}^{N} \lambda^{k+r}(-1)^{k} B\left(f, \dot{T}_{n}^{r+k} h\right) / k ! r ! \\
& =B(f, h)
\end{aligned}
$$

for all $f, h \in S$ and where we used $B\left(\dot{T}_{n} f, h\right)=-B\left(f, \dot{T}_{n} h\right)$. 
The decomposition $L_{n}=\dot{L}_{n}+\tilde{L}_{n}$ may lend itself to stronger selection criteria than the usual condition

$$
\left(L_{n}-\delta_{n, 0} \alpha(0)\right) \psi=0 \quad \forall n \geqq 0
$$

for the physical vectors, e.g.

$$
\begin{aligned}
& L_{0} \psi=\alpha(0) \psi, \\
& \dot{L}_{n} \psi=0=\tilde{L}_{n} \psi \quad \forall n \geqq 1 .
\end{aligned}
$$

Observe that in the transition from $N_{n}, M_{n}$ to $T_{n}, R_{n}$, we have lost the central term of the Virasoro relations, so this information may need to be put back into the theory at the abstract level, and furthermore, to complete the presentation of the physics, we also need to include the Poincaré transformations. However, an easy calculation of $\left[M^{\mu \nu}, \Phi(f)\right]$ shows that the Poincare transformations act as the identity on the mode space part $\tilde{l}^{2}$ of $S$, and so since the rest is finite dimensional, these transformations are integrable by power series, and also commute with the constraints. So in the interests of an economic presentation, we henceforth omit the Poincare transformations from the discussion.

It is now necessary to prove implementability of the families $\exp \lambda \bar{X}$, where $X \in\left\{T_{0}, \dot{T}_{n}, \dot{R}_{n}, \tilde{T}_{n}, \tilde{R}_{n} \mid n \in \mathbb{Z}^{*}\right\}$ by weak operator continuous families of $\mathrm{J}$ unitary operators. This will indicate the usefulness of the Fock-representation, in that if these families are implementable, it may be possible to define the integrated constraints from these implementers in an acceptable way.

Theorem 5.7. (i) The families $\exp \lambda \bar{X}$ for $X \in\left\{\tilde{T}_{n}, \tilde{R}_{n} \mid n \geqq 1\right\}$ are implementable on $\Phi$ by weak operator continuous families of $J$-unitary operators, $\Gamma(\exp \lambda \bar{X})$.

(ii) Let $A \in\left\{\dot{N}_{n}, \dot{M}_{n} \mid n \geqq 1\right\}$, then $[A, \Gamma(J)] \chi=0 \forall \chi \in F_{00}$, and $A$ is essentially self-adjoint. Hence by spectral theory we can construct $\exp i \lambda \bar{A}$, and this will be a weak operator continuous one parameter group.

(iii) Given $A=\dot{N}_{n}$ or $\dot{M}_{n}$,

$$
e^{i \lambda \bar{A}} \Phi(h) e^{-i \lambda \bar{A}} \xi=\Phi\left(\sum_{n=0}^{\infty} \frac{\lambda^{n}}{n !} T^{n} h\right) \xi
$$

$\forall \lambda \in \mathbb{R}, h \in S_{0}, \xi \in F_{00}$, where $T=\dot{T}_{n}$ or $\dot{R}_{n}$ respectively, and we already know that $\sum_{n=0}^{\infty} \frac{\lambda^{n}}{n !} T^{n} h$ converges absolutely.

Proof. The proof of (i) is standard, because $\exp \lambda \bar{X}$ are weak operator continuous families of unitary (and J-unitary) operators.

(ii) Recall that $\dot{L}_{n}=\frac{1}{4 \alpha^{\prime}} \sum_{k=1}^{n-1} \alpha_{k} \cdot \alpha_{n-k}+\alpha_{n} \cdot p, \dot{L}_{-n}=\left(\dot{L}_{n}\right)^{+}, \dot{N}_{n}=\dot{L}_{n}+\dot{L}_{-n}$ and $\dot{M}_{n}=i\left(\dot{L}_{n}-\dot{L}_{-n}\right)$, where $n \geqq 0$. Hence $A$ consists of a combination of quadratic monomials in the creators and annihilators, not mixing space-time components, and application of Lemma 3.3 will produce $[A, \Gamma(J)] \chi=0 \forall \chi \in F_{00}$. By construction $A$ is $\mathrm{J}$-symmetric, and this then implies that it is symmetric. That $A$ is essentially self adjoint follows from the next lemma. (Recall that $\left.a^{-}(f)=a(J f), \quad a^{+}(f)=a^{*}(f).\right)$ 
Lemma 5.8. Given an operator of the form:

$$
R=\sum_{i=1}^{l}\left\{a\left(f_{i}\right) \cdot a\left(h_{i}\right)+a^{*}\left(g_{i}\right) \cdot a\left(r_{i}\right)+\mathrm{a}^{*}\left(u_{i}\right) \cdot a^{*}\left(v_{i}\right)\right\},
$$

where $f_{i}, h_{i}, g_{i}, r_{i}, u_{i}, v_{i} \in S_{0}$ are arbitrary, the operator $B=R+R^{*}$ is essentially self-adjoint with $F_{00}$ in its domain.

Proof. By construction $B$ is a symmetric operator which leaves $F_{00} \subset C^{\infty}(B)$ invariant. So we only need to prove that $F_{00}$ consists of analytic vectors for $B$. Note that $B$ is of the form

$$
B=\sum_{i=1}^{L} a^{\emptyset}\left(f_{i}\right) a^{\emptyset}\left(h_{i}\right), \quad f_{i}, h_{i} \in S_{0},
$$

where $a^{\emptyset}(f)$ denotes either $a(f)$ or $a^{*}(f)$. Let $\psi$ be an $n$-particle vector, then by the usual estimate

$$
\left\|a^{\emptyset}\left(f_{i}\right) \ldots a^{\emptyset}\left(f_{k}\right) \psi\right\| \leqq \sqrt{n+1} \ldots \sqrt{n+k}\left(\prod_{i=0}^{k}\left\|f_{i}\right\|\right)\|\psi\|,
$$

we obtain $\left\|B^{N} \psi\right\| \leqq L^{N} \sqrt{n+1} \ldots \sqrt{n+2 N} F^{2 N}\|\psi\|$, where $F=\sup \left\{\left\|f_{i}\right\|\right.$, $\left.\left\|h_{i}\right\| \mid i=1, \ldots L\right\}$. So

$$
\begin{aligned}
\sum_{N=0}^{\infty} \frac{\lambda^{N}}{N !}\left\|B^{N} \psi\right\| & \leqq \sum_{N=0}^{\infty} \frac{\left(L F^{2} \lambda\right)^{N}}{N !} \sqrt{n+1} \ldots \sqrt{n+2 N}\|\psi\| \\
& =: \sum_{N=0}^{\infty} c_{N} \cdot\|\psi\| .
\end{aligned}
$$

Apply the ratio test:

$$
\left|\frac{c_{N+1}}{c_{N}}\right|=\frac{L F^{2} \lambda}{N+1} \sqrt{(n+2 N+1)(n+2 N+2)} \rightarrow 2 L F^{2} \lambda
$$

as $N \rightarrow \infty$, and so the series converges for $\lambda<1 / 2 L F^{2}$. Thus $\psi$ is analytic for $B$, and as $F_{00}$ is spanned by the finite particle vectors, $B$ is essentially selfadjoint by Nelson's theorem.

Proof of (iii). We collect a few facts from Stone's theorem for use below: $t \rightarrow e^{i t \bar{A}}$ is weak operator continuous, i.e. $\left\langle\eta,\left(e^{i t \bar{A}}-e^{i s \bar{A}}\right) \xi\right\rangle \underset{t}{\stackrel{s}{\rightarrow}} 0 \forall \eta, \xi \in \mathscr{F}(S)$, and

$$
\lim _{t \rightarrow 0}\left\|\left[t^{-1}\left(e^{i t \bar{A}}-1\right)-i \bar{A}\right] \xi\right\|=0 \quad \forall \xi \in D(\bar{A}) \supseteq F_{00},
$$

from which the Cauchy-Schwarz inequality implies:

$$
\lim _{t \rightarrow 0}\left\langle\eta,\left[t^{-1}\left(e^{i t \bar{A}}-1\right)-i \bar{A}\right] \xi\right\rangle=0 \quad \forall \xi \in D(\bar{A}), \eta \in \mathscr{F}(S) .
$$

Our main argument in this part is aimed at establishing

$$
\left\langle\eta, e^{i t \bar{A}} \Psi(h) e^{-i t \bar{A}} \xi\right\rangle=\sum_{n=0}^{\infty}\left\langle\eta,(\operatorname{ad} i \bar{A})^{n}(\Psi(h)) \xi\right\rangle \frac{t^{n}}{n !}
$$


for all $\eta, \xi \in F_{00}$ and all $t$ in some neighbourhood of zero. Start with the unitary $W(f)=\exp i \Psi(f)$ and define for fixed $\xi \in F_{00}, \eta \in D(\bar{A})$ :

$$
f_{h}(t):=\left\langle\eta, e^{i t \bar{A}} W(h) e^{-i t \bar{A}} \xi\right\rangle, \quad \forall t \in \mathbb{R}, h \in S_{0}
$$

and so:

$$
\begin{aligned}
f_{h}^{\prime}(t)= & \lim _{k \rightarrow 0} k^{-1}\left\langle\eta,\left[e^{i(t+k) \bar{A}} W(h) e^{-i(t+k) \bar{A}}-e^{i t \bar{A}} W(h) e^{-i t \bar{A}}\right] \xi\right\rangle \\
= & \lim _{k \rightarrow 0} k^{-1}\left\langle e^{-i t \bar{A}} \eta,\left[\left(e^{i k \bar{A}}-1\right) W(h)\left(e^{-i k \bar{A}}-1\right)+\left(e^{i k \bar{A}}-1\right) W(h)\right.\right. \\
& \left.\left.+W(h)\left(e^{-i k \bar{A}}-1\right)\right] e^{-i t \bar{A}} \xi\right\rangle \\
= & \lim _{k \rightarrow 0} k^{-1}\left\langle e^{-i t \bar{A}} \eta,\left(e^{i k \bar{A}}-1\right) W(h)\left(e^{-i k \bar{A}}-1\right) \xi\right\rangle \\
& +\lim _{k \rightarrow 0} k^{-1}\left\langle\left(e^{-i k \bar{A}}-1\right) \eta, e^{i t \bar{A}} W(h) e^{-i t \bar{A}} \xi\right\rangle \\
& +\lim _{k \rightarrow 0} k^{-1}\left\langle e^{i t \bar{A}} W(-h) e^{-i t \bar{A}} \eta,\left(e^{-i k \bar{A}}-1\right) \xi\right\rangle \\
= & \left\langle-i \bar{A} \eta, e^{i t \bar{A}} W(h) e^{-i t \bar{A}} \xi\right\rangle+\left\langle e^{i t \bar{A}} W(-h) e^{-i t \bar{A}} \eta,-i \bar{A} \xi\right\rangle,
\end{aligned}
$$

where we made use of:

$$
\begin{aligned}
\lim _{k \rightarrow 0} k^{-1} & \left\langle e^{-i t \bar{A}} \eta,\left(e^{i k \bar{A}}-1\right) W(h)\left(e^{-i k \bar{A}}-1\right) e^{-i t \bar{A}} \xi\right\rangle \\
& \leqq \lim _{k \rightarrow 0} k^{-1}\left\|\left(e^{-i k \bar{A}}-1\right) \eta\right\| \cdot\left\|W(h)\left(e^{-i k \bar{A}}-1\right) e^{-i t \bar{A}} \xi\right\| \\
& =\lim _{k \rightarrow 0} k^{-1}\left\|\left(e^{-i k \bar{A}}-1\right) \eta\right\| \cdot\left\|\left(e^{-i k \bar{A}}-1\right) \xi\right\| \\
& =\lim _{k \rightarrow 0} k^{-1}\left\|\left(e^{-i k \bar{A}}-1\right) \eta\right\| \cdot \lim _{k \rightarrow 0}\left\|\left(e^{-i k \bar{A}}-1\right) \xi\right\| \\
& =0
\end{aligned}
$$

because both the limits in the penultimate step exist. Now since we cannot be sure that $\left(\operatorname{Ad} e^{i t \bar{A}}\right)(W(h))$ preserves $D(\bar{A})$, we do not make use of the self-adjointness of $\bar{A}$ to simplify the equation for $f_{h}^{\prime}(t)$. Instead, introduce the concept

$$
\left\langle\eta,\left(\operatorname{ad}^{w} B\right)(C) \xi\right\rangle:=\left\langle B^{*} \eta, C \xi\right\rangle-\langle\eta, C B \xi\rangle,
$$

where $\eta, \xi \in F_{00}$ and $B, B^{*}$ are operators preserving $F_{00}$, which because it is in $D(C)$, means that $\left\langle\eta,\left(\operatorname{ad}^{w} \mathrm{~B}\right)^{n}(C) \xi\right\rangle$ also makes sense for $n$ a positive integer. Then:

$$
f_{h}^{\prime}(t)=\left\langle\eta,\left(\operatorname{ad}^{w} i \bar{A}\right)\left(e^{i t \bar{A}} W(h) e^{-i t \bar{A}}\right) \xi\right\rangle,
$$

and since $\bar{A}$ preserves $F_{00}$, the $n^{\text {th }}$ derivative

$$
f_{h}^{(n)}(t)=\left\langle\eta,\left(\operatorname{ad}^{w} i \bar{A}\right)^{n}\left(e^{i t \bar{A}} W(h) e^{-i t \bar{A}}\right) \xi\right\rangle
$$

makes sense, and so $f_{h}(t)$ is $C^{\infty}$ as a function of $t$. When $t=0, \operatorname{ad}^{w} i \bar{A}$ becomes just ad $i \bar{A}$ (making use of the self-adjointness of $\bar{A}$ ), i.e...

$$
f_{h}^{(n)}(0)=\left\langle\eta,(\operatorname{ad} i \bar{A})^{n}(W(h)) \xi\right\rangle .
$$

Now the map $s \rightarrow W(s h)$ for $s \in \mathbb{R}$ and for $h \in S_{0}$ fixed, is weak operator continuous by Stone's theorem, so the function $s \rightarrow f_{s h}^{(n)}(0)$ is continuous. We prove that it is 
differentiable at $s=0$ using the fact that $A F_{00} \subseteq F_{00} \subseteq D(\Psi(h))$ :

$$
\begin{aligned}
\left.\frac{d f_{s h}^{(n)}(0)}{d s}\right|_{s=0} & =\left.\lim _{k \rightarrow 0} k^{-1}\left\langle\eta,(\operatorname{ad} i \bar{A})^{n}[W((s+k) h)-W(\operatorname{sh})] \xi\right\rangle\right|_{s=0} \\
& =\left\langle\eta,(\operatorname{ad} i A)^{n}(i \Psi(h)) \xi\right\rangle .
\end{aligned}
$$

Since $f_{h}$ is $C^{\infty}$ in $t$, we can write down its Taylor series in $t$ around 0 , but need to prove convergence for it.

$$
\begin{aligned}
f_{h}(t) & =\sum_{n=0}^{\infty} \frac{f_{h}^{(n)}(0)}{n !} t^{n}=\sum_{n=0}^{\infty}\left\langle\eta,(\operatorname{ad} i \bar{A})^{n}(W(h)) \xi\right\rangle \frac{t^{n}}{n !} \\
\left|f_{h}(t)\right| & \leqq \sum_{n=0}^{\infty} \frac{|t|^{n}}{n !} \sum_{k=0}^{n}\left(\begin{array}{l}
n \\
k
\end{array}\right)\left|\left\langle\bar{A}^{n-k} \eta, W(h) \bar{A}^{k} \xi\right\rangle\right| \\
& \leqq \sum_{n=0}^{\infty}|t|^{n} \sum_{k=0}^{n} \frac{\left\|\bar{A}^{n-k} \eta\right\| \cdot\left\|\bar{A}^{k} \xi\right\|}{k !(n-k) !} \\
& =\lim _{N \rightarrow \infty} \sum_{\substack{m=0 \\
m=0}}^{m=N}\left(\frac{|t|^{m}\left\|\bar{A}^{m} \eta\right\|}{m !}\right) \cdot\left(\frac{|t|^{k}\left\|\bar{A}^{k} \xi\right\|}{k !}\right) \\
& \leqq \lim _{N \rightarrow \infty} \sum_{m=0}^{N} \frac{|t|^{m}\left\|\bar{A}^{m} \eta\right\|}{m !} \cdot \lim _{M \rightarrow \infty} \sum_{k=0}^{M} \frac{|t|^{k}\left\|\bar{A}^{k} \xi\right\|}{k !}
\end{aligned}
$$

For $\xi \in V^{(m)} \cap F_{00}$ we make use of the estimate:

$$
\left\|a^{ \pm}\left(f_{1}\right) \ldots a^{ \pm}\left(f_{k}\right) \xi\right\| \leqq \sqrt{(m+1) \ldots(m+k)}\left\|f_{1}\right\| \ldots\left\|f_{k}\right\| \cdot\|\xi\|,
$$

and to obtain estimates for $\left\|\bar{A}^{k} \xi\right\|$, observe that

$$
\begin{aligned}
\left\|\dot{L}_{n} \xi\right\| & \leqq \frac{1}{4 \alpha^{\prime}} \sum_{k=1}^{n-1}\left\|\alpha_{k} \cdot \alpha_{n-k} \xi\right\|+\left\|\alpha_{n} \cdot p \xi\right\| \\
& \leqq \frac{1}{4 \alpha^{\prime}} \sum_{k=1}^{n-1}(D+1) \sqrt{(m+1)(m+2)}\|\xi\|+\sqrt{2}(D+1) \sqrt{(m+1)(m+2)}\|\xi\| \\
& =\left(\frac{1}{4 \alpha^{\prime}} \frac{n}{2}(n-1)+\sqrt{2}\right)(D+1) \sqrt{(m+1)(m+2)}\|\xi\| \\
& =: \theta \sqrt{(m+1)(m+2)}\|\xi\| .
\end{aligned}
$$

This estimate also holds for $\left\|\dot{L}_{n}^{+} \xi\right\|$, and hence:

$$
\begin{aligned}
\|\bar{A} \xi\| & \leqq 2 \theta \sqrt{(m+1)(m+2)}\|\xi\|, \\
\left\|\bar{A}^{k} \xi\right\| & \leqq(2 \theta)^{k} \sqrt{(m+1)(m+2) \ldots(m+2 k)}\|\xi\|, \\
\sum_{k=0}^{N} \frac{|t|^{k}\left\|\bar{A}^{k} \xi\right\|}{k !} & \leqq \sum_{k=0}^{N}(|t| 2 \theta)^{k} \frac{\sqrt{(m+1) \ldots(m+2 k)}}{k !}\|\xi\|=: \sum_{k=0}^{N} a_{k} .
\end{aligned}
$$


Apply the ratio test for convergence:

$$
\left|\frac{a_{k+1}}{a_{k}}\right|=|2 \theta t| \frac{\sqrt{(m+2 k+1)(m+2 k+2)}}{k+1} \underset{\infty}{\stackrel{k}{\rightarrow}} 2|2 \theta t|,
$$

and is less than one whenever

$$
|t|<1 / 4 \theta=\frac{1}{4}\left[\left(\frac{1}{4 \alpha^{\prime}} \frac{n}{2}(n-1)+\sqrt{2}\right)(D+1)\right]^{-1}
$$

and this will also hold for the series $\sum_{k=0}^{M}|t|^{k}\left\|\bar{A}^{k} \eta\right\| / k$ !, and so the Taylor series converges absolutely for $t$ in the indicated range around 0 . Since $W(h)$ disappears from the estimates, the series of $f_{s h}(t)$ also converges uniformly as functions of $s$, and similarly the series

$$
\sum_{n=0}^{\infty} \frac{d}{d s}\left(\frac{f_{s h}^{(n)}(0)}{n !}\right) t^{n}=\sum_{n=0}^{\infty}\left\langle\eta,(\operatorname{ad} i \bar{A})^{n}(i \Psi(h) W(s h)) \xi\right\rangle \frac{t^{n}}{n !}
$$

will also converge uniformly as a series of functions in $s$, so we can interchange differentiation in $s$ with the limit of the series to obtain, when $|t|<1 / 4 \theta$ :

$$
\begin{aligned}
\left.\frac{d}{d s} f_{s h}(t)\right|_{s=0} & =\sum_{n=0}^{\infty}\left\langle\eta,(\operatorname{ad} i \bar{A})^{n}(i \Psi(h)) \xi\right\rangle \frac{t^{n}}{n !} \\
& =\left.\frac{d}{d s}\left\langle\eta, e^{i t \bar{A}} W(s h) e^{-i t \bar{A}} \xi\right\rangle\right|_{s=0},
\end{aligned}
$$

using the definition of $f_{h}(t)$. Since by the convergence of the series we know that this exists, Stone's theorem implies that $e^{-i t \bar{A}} \xi \in D(\Psi(h))$ for $t$ in the indicated range, and so by application of Stone's theorem:

$$
\begin{aligned}
\left.\frac{d}{d s} f_{\text {sh }}(t)\right|_{s=0} & =\left\langle\eta, e^{i t \bar{A}} i \Psi(h) e^{-i t \bar{A}} \xi\right\rangle, \quad \text { i.e.: } \\
\left\langle\eta, e^{i t \bar{A}} \Psi(h) e^{-i t \bar{A}} \xi\right\rangle & =\sum_{n=0}^{\infty}\left\langle\eta,(\operatorname{ad} i \bar{A})^{n}(\Psi(h)) \xi\right\rangle \frac{t^{n}}{n !}
\end{aligned}
$$

for all $\eta, \xi \in F_{00}$ and $t<1 / 4 \theta$. This corresponds to the usual formal physicist's equation mentioned in Sect. 4. Using $\Phi(h)=\Psi(P-h)-K \Psi\left(K P_{+} h\right)$, the same relation will also hold for $\Phi(h)$ instead of $\Psi(h)$, i.e.

$$
\begin{aligned}
\left\langle\eta, e^{i t \bar{A}} \Phi(h) e^{-i t \bar{A}} \xi\right\rangle & =\sum_{n=0}^{\infty}\left\langle\eta,(\operatorname{ad} i \bar{A})^{n}(\Phi(h)) \xi\right\rangle \frac{t^{n}}{n !} \\
& =\left\langle\eta, \Phi\left(\sum_{k=0}^{\infty} \frac{t^{k}}{k !} T^{k} h\right) \xi\right\rangle
\end{aligned}
$$

where $T=\dot{T}_{n}$ or $\dot{R}_{n}$, and $\eta, \xi \in F_{00},|t|<1 / 4 \theta$. But since $\sum_{n=0}^{\infty} \frac{t^{n}}{n !} T^{n} h \in S_{0}$ for $h \in S_{0}$, this can be repeatedly applied to get

$$
\left\langle\eta, e^{i t \bar{A}} \Phi(h) e^{-i t \bar{A}} \xi\right\rangle=\left\langle\eta, \Phi\left(\sum_{n=0}^{\infty} \frac{t^{n}}{n !} T^{n} h\right) \xi\right\rangle \quad \forall t \in \mathbb{R}, \eta, \xi \in F_{00} .
$$


Since $F_{00}$ separates $\mathscr{F}(S)$, this means that

$$
e^{i t \bar{A}} \Phi(h) e^{-i t \bar{A}} \xi=\Phi\left(\sum_{n=0}^{\infty} \frac{t^{n}}{n !} T^{n} h\right) \xi \quad \forall \xi \in F_{00} .
$$

Note that the convergence proof above showed that $F_{00}$ is a set of analytic vectors for $A$. The proof above will break down at this point for $A=N_{n}$ or $M_{n}$, since it is easily seen that $F_{00}$ is not a subset of the set of analytic vectors of these $A$, and so the Taylor series will not converge (this is because for these $A$, there is an infinite sum in the expression, whose size is limited by the number of nonzero modes of $\xi \in F_{00}$, and since $A$ increases both particle number and maximum mode space number, the exponential sum diverges).

We are now ready to prove both integrability for the full gauge transformations $T_{n}$ and $R_{n}$ as well as implementability for the integrated transformations. Denote by $\operatorname{Sp}(S, B)$ the group of symplectic transformations on $\{S, B\}$.

Theorem 5.9. (i) The limits

$$
\begin{aligned}
& \text { s-lim }\left(\exp \frac{t}{k} \overline{\tilde{T}}_{n} \cdot \exp \frac{t}{k} \dot{T}_{n}\right)^{k} f=: G_{t}^{n} f, \quad f \in S, \\
& \mathrm{~s}-\lim _{k \rightarrow \infty}\left(\exp \frac{t}{k} \overline{\tilde{R}}_{n} \cdot \exp \frac{t}{k} \dot{R}_{n}\right)^{k} f=: Q_{t}^{n} f, \quad f \in S
\end{aligned}
$$

exist in the strong Hilbert space topology on $\left(S,\langle\cdot, \cdot\rangle_{K}^{J}\right)$ and

$$
\begin{aligned}
e^{i t \bar{N}_{n}} \Phi(f) e^{-i t \bar{N}_{n}} \psi=\Phi\left(G_{t}^{n} f\right) \psi & \forall f \in S_{0}, \psi \in F_{00}, \\
e^{i t \bar{M}_{n}} \Phi(f) e^{-i t \bar{M}_{n}} \psi=\Phi\left(Q_{t}^{n} f\right) \psi & \forall f \in S_{0}, \psi \in F_{00} .
\end{aligned}
$$

(ii) $G_{t}^{n}, \quad Q_{t}^{n} \in \operatorname{Sp}^{J}(S, B):=\{T \in \operatorname{Sp}(S, B) \mid[T, J]=0\}, \quad\left[G_{t}^{n}, K\right] \neq 0 \neq\left[Q_{t}^{n}, K\right]$, and $t \rightarrow G_{t}^{n}$ and $t \rightarrow Q_{t}^{n}$ are one parameter groups for all $n \in \mathbb{Z}^{*}$.

Proof. (i) Begin by collecting some relevant facts and notation. Fix $n \in \mathbb{Z}^{*}$. $S_{t}:=\exp t \tilde{T}_{n}, T_{t}:=\exp t \dot{T}_{n} \in \operatorname{Sp}^{J}(S, B) . U_{t}:=\Gamma\left(S_{t}\right), V_{t}:=\exp i t \dot{N}_{n}$ and by 5.7 we have $U_{t} \Phi(f) U_{-t} \xi=\Phi\left(S_{t} f\right) \xi$ and $V_{t} \Phi(f) V_{-t} \xi=\Phi\left(T_{t} f\right) \xi \forall f \in S_{0}, \xi \in F_{00}$.

Lemma 5.10. $U_{t}=\Gamma\left(\exp t \tilde{T}_{n}\right)=\exp$ it $\tilde{N}_{n}$.

Proof. $\Gamma\left(\exp t \tilde{T}_{n}\right)=\exp t d \Gamma\left(\tilde{T}_{n}\right)$. Choose a generic $m$-particle vector: $\psi=$ $\prod_{i=1}^{m} a^{*}\left(f_{i}\right) \Omega \in V^{(m)}$, then

$$
\begin{aligned}
i \tilde{N}_{n} \psi= & {\left[i \tilde{N}_{n}, a^{*}\left(f_{1}\right)\right] \prod_{i=2}^{m} a^{*}\left(f_{i}\right) \Omega+a^{*}\left(f_{1}\right) i \tilde{N}_{n} \prod_{i=2}^{m} a^{*}\left(f_{i}\right) \Omega } \\
= & a^{*}\left(\tilde{T}_{n} f_{1}\right) \prod_{i=2}^{m} a^{*}\left(f_{i}\right) \Omega+a^{*}\left(f_{1}\right)\left[i \tilde{N}_{n}, a^{*}\left(f_{2}\right)\right] \prod_{i=3}^{m} a^{*}\left(f_{i}\right) \Omega \\
& +a^{*}\left(f_{1}\right) a^{*}\left(f_{2}\right) i \tilde{N}_{n} \prod_{i=3}^{m} a^{*}\left(f_{i}\right) \Omega \\
= & \ldots=d \Gamma\left(\tilde{T}_{n}\right) \psi .
\end{aligned}
$$


Since it is inconvenient that $\Phi(f)$ exponentiates up to an unbounded operator, we now switch our attention to the auxiliary field $\Psi(f)$, using the relation $\Phi(f)=\Psi\left(P_{-} f\right)-K \Psi\left(K P_{+} f\right)$, where $J=P_{+}-P_{-}$. We also use the notation $B_{J}(\cdot, \cdot):=B(\cdot, J \cdot)$.

Lemma 5.11. (i) There is a group isomorphism $\theta: \operatorname{Sp}^{J}(S, B) \rightarrow \operatorname{Sp}^{J}\left(S, B_{J}\right)$ given by $\theta(T):=P_{-} T-P_{+} K T K \forall T \in \operatorname{Sp}^{J}(S, B)$.

(ii) Given $T \in \operatorname{Sp}^{J}(S, B)$ and unitaries $U_{T}$ such that $\left[U_{T}, \Gamma(J)\right]=0$, then

$$
U_{T} \Phi(f) U_{T}^{-1} \chi=\Phi(T f) \chi \quad \forall f \in S, \chi \in F_{0}
$$

iff

$$
U_{T} \Psi(f) U_{T}^{-1} \chi=\Psi(\theta(T)) \chi \quad \forall f \in S, \chi \in F_{00} .
$$

Proof. (i) Since the inverse of $\theta$ is again the same expression:

$\theta^{-1}(T)=P_{-} T-P_{+} K T K \forall T \in \operatorname{Sp}^{J}\left(S, B_{J}\right)$ (making use of $\left[K, P_{ \pm}\right]=0$ ), we only need to show it is a homomorphism,

$$
\begin{aligned}
\theta\left(T_{1} T_{2}\right) & =P_{-} T_{1} T_{2}-P_{+} K T_{1} T_{2} K=P_{-} T_{1} P_{-} T_{2}+P_{+} K T_{1} K \cdot P_{+} K T_{2} K \\
& =\left(P_{-} T_{1}-P_{+} K T_{1} K\right) \cdot\left(P_{-} T_{2}-P_{+} K T_{2} K\right)=\theta\left(T_{1}\right) \cdot \theta\left(T_{2}\right),
\end{aligned}
$$

where use was made of $\left[T_{i}, P_{ \pm}\right]=0, i=1,2$ which follows from $\left[T_{i}, J\right]=0$.

(ii) Given $T, U_{T}$ and $U_{T} \Phi(f) U_{T}^{-1} \chi=\Phi(T f) \chi$ we calculate:

$$
\begin{aligned}
U_{T} \Phi(f) U_{T}^{-1} \chi & =U_{T} \Psi\left(P_{-} f\right) U_{T}^{-1} \chi-K U_{T} \Psi\left(K P_{+} f\right) U_{T}^{-1} \chi \\
& =\Phi(T f) \chi=\Psi\left(T P_{-} f\right) \chi-K \Psi\left(K T P_{+} f\right) \chi .
\end{aligned}
$$

But since we can vary the components $P_{ \pm} f$ of $f$ independently:

$$
\begin{aligned}
U_{T} \Psi\left(P_{-} f\right) U_{T}^{-1} \chi & =\Psi\left(T P_{-} f\right) \chi \quad \text { and } \\
U_{T} \Psi\left(K P_{+} f\right) U_{T}^{-1} \chi & =\Psi\left(K T P_{+} f\right) \chi \quad \text { which implies that } \\
U_{T} \Psi\left(P_{+} f\right) U_{T}^{-1} \chi & =\Psi\left(-K T K P_{+} f\right) \chi, \quad \text { using }\left[K, P_{ \pm}\right]=0 . \\
\text { So } \quad U_{T} \Psi(f) U_{T}^{-1} \chi & =\Psi\left(\left(P_{-} T-P_{+} K T K\right) f\right) \chi=\Psi(\theta(T) f) \chi
\end{aligned}
$$

and since this argument is reversible, the lemma is proved.

We now intend to use the Trotter formula (cf. Reed and Simon [26]):

$$
\lim _{k \rightarrow \infty}\left(U_{t / k} V_{t / k}\right)^{k}=\exp i t \overline{\left(\tilde{N}_{n}+\dot{N}_{n}\right)}=\exp i t \bar{N}_{n},
$$

where the limit is in the strong operator topology and $N_{n}, \dot{N}_{n}$ and $\tilde{N}_{n}$ all are essentially selfadjoint by Lemma 5.8. Denote $W(f):=\exp i \Psi(f)$. Now

$$
e^{i t \bar{N}_{n}} W(f) e^{-i t \bar{N}_{n}} \psi=\mathrm{s}-\operatorname{lims}_{m \rightarrow \infty} \lim \left(U_{t / m} V_{t / m}\right)^{m} W(f)\left(V_{-t / k} U_{-t / k}\right)^{k} \psi
$$

for all $\psi \in \mathscr{F}(S)$. We now use the following fact (easy to prove and standard):

Lemma 5.12. Given two strong operator convergent sequences of unitaries $A_{m} \rightarrow A$, $B_{m} \rightarrow B$ on $\mathscr{F}(S)$, we have $\mathrm{s}-\lim _{m \rightarrow \infty} A_{m} B_{m} \psi=A B \psi$ for all $\psi \in \mathscr{F}(S)$. That is,

$$
\mathrm{s}-\lim _{m \rightarrow \infty} \mathrm{s}-\lim _{k \rightarrow \infty} A_{m} B_{k} \psi=A B \psi=\operatorname{s}_{m \rightarrow \infty} \lim _{m} B_{m} \psi \text {. }
$$


So application of 5.12 to Eq. (1) above produces:

$$
\begin{aligned}
e^{i t \bar{N}_{n}} W(f) e^{-i t \bar{N}_{n}} \psi= & \operatorname{s-lim}\left(U_{t / m} V_{t / m}\right)^{m} W(f)\left(V_{-t / m} U_{-t / m}\right)^{m} \psi \\
= & s-\lim _{m \rightarrow \infty} W\left(\left[\theta^{-1}\left(S_{t / m}\right) \theta^{-1}\left(T_{t / m}\right)\right]^{m} f\right) \psi \\
& =s-\lim _{m \rightarrow \infty} W\left(\theta^{-1}\left(\left(S_{t / m} T_{t / m}\right)^{m}\right) f\right) \psi,
\end{aligned}
$$

where we made use of $U_{t} \Psi(f) U_{-t} \psi=\Psi\left(\theta^{-1}\left(S_{t}\right) f\right) \psi$ and $V_{t} \Psi(f) V_{-t} \psi=$ $\Psi\left(\theta^{-1}\left(T_{t}\right)\right) \psi$ which followed from 5.11(ii) and the listed relations at the start of this proof.

Lemma 5.13. Let $\left\{f_{m}\right\} \subset S$ be a sequence and assume that the limit $\mathrm{s}-\lim _{m \rightarrow \infty} W\left(f_{m}\right) \psi$ exists for all $\psi \in \mathscr{F}(S)$. Then $\mathrm{s}-\lim _{m \rightarrow \infty} f_{m}$ exists and

$$
\begin{aligned}
& \operatorname{s-lim}_{m \rightarrow \infty} W\left(f_{m}\right) \psi=W\left(\operatorname{s-lim}_{m \rightarrow \infty} f_{m}\right) \psi \\
& \mathrm{s}-\lim _{m \rightarrow \infty} \Psi\left(f_{m}\right) \psi=\Psi\left({ }_{\mathrm{s}-\lim _{m \rightarrow \infty} f_{m}}\right) \psi \quad \forall \psi \in F_{0} .
\end{aligned}
$$

Proof. By Reed and Simon X41(d) [26], the last two statements follow from the existence of $s-\lim _{m \rightarrow \infty} f_{m}$, so we only need to prove this existence. Since $\mathrm{s}-\lim _{m \rightarrow \infty} W\left(f_{m}\right) \psi$ exist by hypothesis, and the exponential series $W(f) \psi=\sum_{n=0}^{\infty}[\Psi(f)]^{n} \psi / n$ ! converges absolutely in the Hilbert norm for all $\psi \in F_{0}$, we can interchange a limit with the limit of the series to obtain for all $\psi \in F_{0}$ :

$$
\begin{aligned}
\operatorname{s-lim} W\left(\lambda f_{m}\right) \psi & =\sum_{k=0}^{\infty} \frac{\lambda^{k}}{k !} \mathrm{s}-\lim \left[\Psi\left(f_{m}\right)\right]^{k} \psi, \text { so } \\
\frac{d}{d \lambda} \mathrm{s}-\left.\lim W\left(\lambda f_{m}\right) \psi\right|_{\lambda=0} & =\operatorname{s-lim}_{m \rightarrow \infty} \Psi\left(f_{m}\right) \psi
\end{aligned}
$$

exists. Now $\Psi\left(f_{m}\right) \Omega=\frac{1}{\sqrt{2}}\left(a\left(f_{m}\right)+a^{*}\left(f_{m}\right)\right) \Omega=\frac{1}{\sqrt{2}} f_{m} \in V^{(1)}=S, \quad$ hence s- $\lim _{m \rightarrow \infty} \Psi\left(f_{m}\right) \Omega=\frac{1}{\sqrt{2}} \mathrm{~s}-\lim f_{m}$ exists.

Now apply 5.13 to Eq. (2) to prove that the limit

$$
\begin{gathered}
\mathrm{s}-\lim _{m \rightarrow \infty} \theta^{-1}\left(\left(S_{t / m} T_{t / m}\right)^{m}\right) f=: \theta^{-1}\left(G_{t}^{n}\right) f \quad \text { exists, and that } \\
e^{i t \bar{N}_{n}} W(f) e^{-i t \bar{N}_{n}} \psi=W\left(\theta^{-1}\left(G_{t}^{n}\right) f\right) \psi \quad \text { and hence: } \\
e^{i t \bar{N}_{n}} \Psi(f) e^{-i t \bar{N}_{n}} \psi=\Psi\left(\theta^{-1}\left(G_{t}^{n}\right) f\right) \psi, \quad \psi \in F_{0} .
\end{gathered}
$$

Thus from Lemma 5.11, $e^{i t \bar{N}_{n}} \Phi(f) e^{-i t \bar{N}_{n}} \psi=\Phi\left(G_{t}^{n} f\right) \psi, \psi \in F_{0}$. Since all operators in the definition of $\theta$ are Hilbert norm continuous, $G_{t}^{n} f=\mathrm{s}-\lim _{m \rightarrow \infty}\left(S_{t / m} T_{t / m}\right) f$ and this completes the proof of 5.9(i), given that $Q_{t}^{n}$ can be treated in complete analogy. 
Proof of 5.9(ii). $G_{t}^{n}, Q_{t}^{n} \in \mathrm{Sp}^{J}(S, B)$ follows from the facts that $U_{t}, V_{t}, e^{t \tilde{R}_{n}}$, $e^{t \dot{R}_{n}} \in \operatorname{Sp}^{J}(S, B)$ and the symplectic form is continuous w.r.t. the Hilbert space topology (alternatively use the CCR's of $\Phi(f)$ and the implementing equations in 5.9(i).)

To see that $\left[G_{t}^{n}, K\right] \neq 0 \neq\left[Q_{t}^{n}, K\right]$, differentiate the implementing equations in 5.9(i) to obtain

$$
\left.\frac{d}{d t} \Phi\left(G_{t}^{n} f\right) \psi\right|_{t=0}=\left[i \bar{N}_{n}, \Phi(f)\right] \psi=\Phi\left(T_{n} f\right) \psi
$$

and we already know that $\left[T_{n}, K\right] \neq 0$.

From $e^{i t \bar{N}_{n}} e^{i s \bar{N}_{n}}=e^{i(s+t) N_{n}}$ and the implementation equations in 5.9(i) we also obtain $G_{t}^{n} G_{s}^{n}=G_{t+s}^{n} \forall t, s \in \mathbb{R}, G_{0}^{n}=I$ and similarly for $Q_{t}^{n}$.

So we may regard $G_{t}^{n}$ and $Q_{t}^{n}$ as the integrated gauge transformations for the string, given that they have the appropriate implementers. These will not be contractions for all values of $t$ (since $\exp t \dot{T}_{n}$ is not). So finally, we have obtained as a starting point for the $\mathrm{C}^{*}$-algebra of the string:

(i) a symplectic space $\{S, B\}$ modelling the behaviour of the excitation modes $\alpha_{n}$,

(ii) a one parameter symplectic group $\exp \lambda T_{0}$ giving time evolution for the string,

(iii) a collection of one parameter families of symplectic groups

$$
\left\{G_{t}^{n}, Q_{t}^{n} \mid n \in \mathbb{Z}^{*}\right\}
$$

giving the gauge transformations of the string,

(iv) the Poincare group acting as symplectic transformations on $S$.

The central term of the Virasoro relations is regarded as a representation dependent phenomenon, as is the use of Krein spaces. In the next section we will consider these issues in detail.

To obtain a full theory for the string, including gauge transformations, we have two options:

(i) Consider the Fock-representation as physically unimportant, an historical accident, and set up the algebraic part of the theory in a purely $\mathrm{C}^{*}$-algebraic framework - a representation-free formalism. We intend to do this in the next section, and also to find a criterion for selecting the physical representations. We do not expect the Fock-representation to satisfy this criterion.

(ii) Consider the Fock-representation as physically important, but in need of augmenting, and obtain the whole orbit of the Fock-representation under the action of the gauge group as is done by Bowick and Rajeev [16, 17] enlarging the field algebra correspondingly. We will explore this option in a future project.

\section{The Field Algebra of the String}

In this section we will propose a $C^{*}$-dynamical system to describe the string, examine its physical representations and constraint conditions. Following that we consider how the Virasoro central term is reintroduced at the algebraic level (if 
required) and study the set of Fock-Krein representations of the string from a $\mathrm{C}^{*}$-algebra point of view.

Given that the string fields $\Phi(f)$ satisfy

$$
[\Phi(f), \Phi(h)] \chi=i B(f, h) \chi \quad \forall f, h \in S, \quad \chi \in F_{0} .
$$

we can use the CCR algebra $\overline{\Delta(S, B)}$ (cf. [9]) to abstract the algebraic structure of these. In this case, there is an action $\alpha$ of the discrete symplectic group $\alpha: \operatorname{Sp}(S, B) \rightarrow$ Aut $\overline{\Delta(S, B)}$ given by $\alpha_{T}\left(\delta_{f}\right):=\delta_{T f} \forall T \in \operatorname{Sp}(S, B), f \in S$, where $\left\{\delta_{f} \mid\right.$ $f \in S\}$ is the set of generating unitaries of $\overline{\Delta(S, B)}$ satisfying the Weyl relations [9]. There is then an invariant state $\omega_{0} \in \mathbb{S}(\overline{\Delta(S, B)})$ for the action $\alpha$, and it is given by $\omega_{0}\left(\delta_{f}\right)=1$ if $f=0$ and $\omega_{0}\left(\delta_{f}\right)=0$ if $f \neq 0$. Now, as remarked at the end of the previous section, all the relevant transformations of the string have been written as symplectic transformations acting on the string field smearing space, so we can use the action $\alpha$ to define these as automorphisms on $\overline{\Delta(S, B)}$. Let $\mathscr{G}$ be the group in $\operatorname{Sp}(S, B)$ generated by the time translations and gauge transformations, i.e. by the set $\left\{e^{t T_{0}}, G_{t}^{n}, Q_{t}^{n} \mid t \in \mathbb{R}, n \in \mathbb{Z}^{*}\right\}$. Let $\mathscr{L} \subset \operatorname{Sp}(S, B)$ be the group of Poincare transformations on $S$, and $G$ is the discrete group generated by both $\mathscr{G}$ and by $\mathscr{L}$. Since $\mathscr{L}$ only acts on the $M^{D+1}$ part of $S=\mathbb{R}^{2} \times M^{D+1} \times \tilde{l}^{2}$ and $\mathscr{G}$ only acts nontrivially on the $\mathbb{R}^{2} \times \tilde{l}^{2}$ part, this means that $G \cong \mathscr{L} \times \mathscr{G}$. We now define the action $\alpha: G \rightarrow \operatorname{Aut} \overline{\Delta(S, B)}$ by the restriction of the action $\alpha$ on $\operatorname{Sp}(S, B)$ above. Our claim is that at the abstract algebraic level all the physical information of the string is contained in the $\mathrm{C}^{*}$-dynamical system $(\alpha, G, \overline{\Delta(S, B)})$.

Now physical representations of the string should be representations of $\overline{\Delta(S, B)}$ which are covariant or at least projective covariant for this dynamical system. This requirement can be easily absorbed into the abstract framework as follows. Start by making a choice of cocycle $\sigma \in Z^{2}(\mathscr{G}, \mathbb{T})$ which we require for the projective representation of $\mathscr{G}$. (E.g. $\sigma=1$ for a covariant system, and if we want specifically the Virasoro central term, then we obtain a nontrivial $\sigma \propto 1$ from the unitaries $e^{i t L_{0}}$, $e^{i t \bar{N}_{n}}, e^{i t \bar{M}_{n}}$ on $\mathscr{F}(S)$ for it.) Extend the cocycle trivially from $\mathscr{G}$ to $G \cong \mathscr{L} \times \mathscr{G}$, and construct the twisted crossed product $\mathscr{F}_{\sigma}:=G \times_{\alpha, \sigma} \overline{\Delta(S, B)}$ (consult Raeburn [30] or Sutherland [31] for its definition). Then $\mathscr{F}_{\sigma}$ has the following properties: there is a canonical injection $i: \overline{\Delta(S, B)} \rightarrow \mathscr{F}_{\sigma}$ as well as a twisted injective homomorphism $u: G \rightarrow U\left(\mathscr{F}_{\sigma}\right) \quad$ such that $u_{g} u_{h}=\sigma(g, h) u_{g h} \quad \forall g, h \in G \quad$ and $\quad i\left(\alpha_{g}(A)\right)=$ $u_{g} i(A) u_{g}^{-1} \forall A \in \overline{\Delta(S, B)}, g \in G$. Moreover $\mathscr{F}_{\sigma}$ has the universal property that its nondegenerate representations are in bijection with the $\sigma$-covariant representations of $\overline{\Delta(S, B)}$ and in such a way that given a $\sigma$-covariant representation $(\pi, V)$, $\pi: \overline{\Delta(S, B)} \rightarrow \mathscr{B}(\mathscr{H}), V: G \rightarrow U(\mathscr{H}), V_{g} V_{h}=\sigma(g, h) V_{g h}, \pi\left(\alpha_{g}(A)\right)=V_{g} \pi(A) V_{g}^{-1}$, then there is a representation $\tilde{\pi}: \mathscr{F}_{\sigma} \rightarrow \mathscr{B}(\mathscr{H})$ for which $\tilde{\pi} \circ i=\pi$ and $V_{g}=\tilde{\pi}\left(u_{g}\right)$ $\forall g \in G$. So $\mathscr{F}_{\sigma}$ is a good candidate for the full field algebra for the string if all physical representations are required to be $\sigma$-covariant.

Now in a representation of $\mathscr{F}_{\sigma}$ we need to select the physical subtheory of the string, and this is done through the enforcement of constraints. Guided by our findings in [19], we will not require that physical states be either regular (i.e. the maps $t \rightarrow \pi\left(\delta_{t f}\right)$ are strong operator continuous for all $f \in S$ ) or that the one parameter unitary groups $t \rightarrow \pi\left(u_{\exp t T_{0}}\right), t \rightarrow \pi\left(u_{G_{t}^{n}}\right), t \rightarrow \pi\left(u_{Q_{t}^{n}}\right)$ be strong operator continuous. So we allow for the fact that gauge generators may not be definable, nor the infinitesimal theory of the string used by physicists in these representations. 
This is to avoid the problems that occur when constraints cannot be enforced because they are eigenvalue equations with the eigenvalue in the continuous part of the spectrum. This problem occurs in the Fock-Krein representation of the string in the preceding sections.

The case $\sigma=1$ is most easily dealt with, and its existence is a bonus of the $\mathrm{C}^{*}$-approach. This corresponds to the absence of the central term in the commutation relations of the heuristic constraints. So at the heuristic level, for such a theory, physical vectors $\psi$ can be selected by

$$
L_{0} \psi=\alpha(0) \psi, \quad N_{n} \psi=0=M_{n} \psi \quad \forall n \in \mathbb{Z}^{*}
$$

or at the exponentiated level:

$$
e^{i t\left(L_{0}-\alpha(0)\right)} \psi=e^{i t \bar{N}_{n}} \psi=e^{i t \bar{M}_{n}} \psi=\psi \quad \forall t \in \mathbb{R}, n \in \mathbb{Z}^{*} .
$$

Now in a given representation $\pi: \mathscr{F}_{\sigma} \rightarrow \mathscr{B}(\mathscr{H})$, we have that $\pi\left(u_{\exp t T_{0}}\right)\left(\right.$ resp. $\pi\left(u_{G_{t}^{n}}\right)$, $\pi\left(u_{Q_{t}^{n}}\right)$ ) correspond to the heuristic objects $e^{i t L_{0}}$ (resp. $\left.e^{i t \bar{N}_{n}}, e^{i t \bar{M}_{n}}\right)$, and this suggests the choice of a $\mathrm{C}^{*}$-constraint set

$$
\mathscr{U}:=\left\{u_{\exp i T_{0}} e^{-i t \alpha(0)}, u_{G_{t}^{n}}, u_{Q_{t}^{n}} \mid t \in \mathbb{R}, n \in \mathbb{Z}^{*}\right\}
$$

as abstract constraints in $\mathscr{F}_{1}$ (cf. $[32,29]$ ) from which the physical states are selected as Dirac states:

$$
\left.\Im_{D}:=\left\{\omega \in S_{\left(\mathscr{F}_{1}\right.}\right) \mid \omega(U)=1 \forall U \in \mathscr{U}\right\}
$$

and each vector state induced by a vector $\psi$ satisfying Eq. (1) in some representation will be in $\varsigma_{D}$. Then $\Im_{D} \neq \emptyset$ iff $\mathbb{1} \notin C^{*}(\mathscr{U}-\mathbb{1})$ (cf. [32]) and in this case $\mathscr{U}$ is called first-class, otherwise it contains second class constraints [33]. Now if $\alpha(0)=0$ it is easy to see that $\mathscr{U}$ is first class: this is because there is already given the $\alpha$-invariant state $\omega_{0}$ on $\overline{\Delta(S, B)}$, and by the technique in the proof of Theorem 3.3 [29], such an invariant state can always be used to construct a Dirac state on $\mathscr{F}_{1}$. If $\alpha(0) \neq 0$, there is an additional phase in the constraint relations, and this may make $\mathscr{U}$ second class. This question calls for detailed analysis of the structure of $\mathscr{U}$. Based on the heuristic findings, we guess that for some values of $\alpha(0), \mathscr{U}$ is second class. This analysis is left for the future.

Next consider the case $\sigma \neq 1$. In this case it is very easy to see that $\mathscr{U}$ is second class, and one may impose the constraints either by using the method proposed in [33] for dealing with quantum second class constraints, or by imitating the heuristic constraint conditions $L_{n} \psi=\alpha(0) \delta_{n, 0} \psi \forall n \geqq 0$. (We do not expect these two methods to give the same answer.) The latter is done as follows. Recall the correspondences $e^{i t L_{0}} \leftrightarrow \pi\left(u_{\exp t T_{0}}\right), e^{i t \bar{N}_{n}} \leftrightarrow \pi\left(u_{G_{i}^{n}}\right), e^{i t \bar{M}_{n}} \leftrightarrow \pi\left(u_{Q_{t}^{n}}\right)$ for a representation $\pi$ of $\mathscr{F}_{\sigma}$. Then we propose selection conditions for physical states by: an $\omega \in \mathfrak{S}\left(\mathscr{F}_{\sigma}\right)$ is in $\mathfrak{S}_{P}$ if

(i) $\omega\left(u_{\exp t T_{0}}\right)=e^{i t \alpha(0)} \forall t \in \mathbb{R}$,

(ii) $\lim _{t \rightarrow 0} t^{-1} \omega\left(A\left[u_{G_{t}^{n}}-i u_{Q_{t}^{n}}+(i-1) \mathbb{1}\right]\right)=0 \quad \forall n \geqq 1, A \in \overline{\Delta(S, B)}$.

(Since in the Fock-representation $\pi_{F}, \bar{N}_{n}$ is the generator of the one parameter group $t \rightarrow \pi_{F}\left(u_{G_{t}^{n}}\right)$ and $\bar{M}_{n}$ generates $t \rightarrow \pi_{F}\left(u_{Q_{t}^{n}}\right)$, and $N_{n}-i M_{n}=2 L_{n}$.)

Whether $\Xi_{P} \neq \emptyset$ we do not know. Our preferred option for dealing with second class constraints is the method of [33] which is somewhat more refined than just the selection of the gauge invariant part of $\overline{\Delta(S, B)}$ as the physical observables. Moreover, even at heuristic level, the splitting of the constraint set in half by the 
Gupta-Bleuler type method used for the string does not get rid of all the gauge degrees of freedom.

Finally, we wish to examine Fock-Krein representations of the string from the $C^{*}$-algebra point of view Since the string field $\Phi(f)$ on the Fock-Krein space $\left\{\mathscr{F}(S),\langle\cdot, \cdot\rangle_{J}\right\}$ exponentiated up to unbounded operators $\exp i \Phi(f)$ (cf. Sect. 3), this forces us to consider $\delta_{f} \rightarrow \overline{\exp i \Phi(f)}$ as an unbounded Krein representation of the *-algebra $\Delta(S, B)$. (Exponentiation of $i \Phi(f)$ is in terms of a power series). However we can use the auxiliary field

$$
\Psi(f)=\Phi\left(P_{-} f\right)-K \Phi\left(K P_{+} f\right), \quad[\Psi(f), \Psi(h)]=i B_{J}(f, h)
$$

(which is modelled by $\overline{\Delta\left(S, B_{J}\right)}$ in an ordinary Fock representation on $\mathscr{F}(S)$ to convert the problem of these Krein representations to a $C^{*}$-algebra one. Also, by Lemma 5.11 it is sufficient for $T \in \operatorname{Sp}^{J}(S, B)$ to study implementability of $\theta(T)$ on $\overline{\Delta\left(S, B_{J}\right)}$ to obtain information of implementability of $T$ on $\Delta(S, B)$ in the FockKrein representation. Note that time evolutions and gauge transformations are all in $\operatorname{Sp}^{J}(S, B)$, but the Poincaré transformations are not. These arguments are easily made into a proof of:

Proposition 6.1. (i) A Fock-Krein representation $\pi_{F}^{J}$ (as in Sect. 3) of $\Delta(S, B)$ is by construction uniquely associated to a Fock-representation $\pi_{F}$ of $\overline{\Delta\left(S, B_{J}\right)}$ on $\mathscr{F}(S)$. (ii) For $T \in \operatorname{Sp}^{J}(S, B)$ and unitaries $U_{T}$ on $\mathscr{F}(S)$ with $\left[U_{T}, \Gamma(J)\right]=0$, we have

iff

$$
U_{T} \pi_{F}^{J}\left(\delta_{f}\right) U_{T}^{-1} \chi=\pi_{F}^{J}\left(\delta_{T f}\right) \quad \forall f \in S, \chi \in F_{0},
$$

$$
U_{T} \pi_{F}\left(\delta_{f}\right) U_{T}^{-1} \chi=\pi_{F}\left(\delta_{\theta(T) f}\right) \chi \quad \forall f \in S, \chi \in F_{0} .
$$

This can be generalised to other regular representations of $\overline{\Delta\left(S, B_{J}\right)}$ and also to other Krein structures $J^{\prime} \neq J$, in which case the auxiliary $C^{*}$-algebra $\overline{\Delta\left(S, B_{J^{\prime}}\right)}$ and dynamical system is different.

We leave the important and difficult problem of the enforcement of the constraints and consequent selection of physical representations for a future investigation. We claim that at this point the aim of the paper that of obtaining an adequate $\mathrm{C}^{*}$-framework for the string - has been achieved.

\section{Appendix}

All the operators here act on and preserve $F_{00}$, and so for ease of notation we do not indicate this explicitly. Recall identifications

$$
a^{-}(\delta(n, \mu))=\alpha_{n}^{\mu}, \quad a^{+}(\delta(n, \mu))=\alpha_{-n}^{\mu}, \quad n \geqq 0
$$

with

$$
\begin{gathered}
{\left[\alpha_{n}^{\mu}, \alpha_{m}^{v}\right]=-2 \alpha^{\prime} n g^{\mu v} \delta_{n+m, 0} \quad \forall n,} \\
L_{0}=\frac{1}{2 \alpha^{\prime}} \sum_{k=1}^{\infty} \alpha_{-k} \cdot \alpha_{k}+\alpha p \cdot p, \\
L_{n}=\frac{1}{4 \alpha^{\prime}} \sum_{k=1}^{n-1} \alpha_{k} \cdot \alpha_{n-k}+\frac{1}{2 \alpha^{\prime}} \sum_{k=1}^{\infty} \alpha_{-k} \cdot \alpha_{n+k}+\alpha_{n} \cdot p,
\end{gathered}
$$


for $n \geqq 1$. Then $\left[L_{n}, p^{\mu}\right]=0$, and using notation $i=K$ for the complex structure:

$\left[L_{n}, q^{\mu}\right]=i \alpha_{n}^{\mu} \quad \forall n, \quad\left[L_{0}, \alpha_{m}^{\mu}\right]=m \alpha_{m}^{\mu} \quad \forall m \neq 0, \quad$ and

$\left[L_{n}, \alpha_{m}^{\mu}\right]=m \alpha_{n+m}^{\mu} \quad \forall n, m$. Recall now:

$$
\begin{aligned}
\Phi(f) & =p\left(f^{(1)}\right)+q\left(f^{(2)}\right) \\
& =p \cdot f_{0}^{(1)}+q \cdot f_{0}^{(2)}+\frac{1}{\sqrt{2}} \sum_{k=1}^{\infty}\left(i\left(\alpha_{-k}-\alpha_{k}\right) \cdot f_{k}^{(1)}+\left(\alpha_{-k}+\alpha_{k}\right) \cdot f_{k}^{(2)}\right) .
\end{aligned}
$$

So $\forall n \in \mathbb{Z}$ :

$$
\begin{aligned}
{\left[L_{n}, \Phi(f)\right] } & =i f_{0}^{(2)} \cdot \alpha_{n}+\frac{1}{\sqrt{2}} \sum_{k=1}^{\infty}\left(\left[L_{n}, \alpha_{-k}\right]\left(f_{k}^{(2)}+i f_{k}^{(1)}\right)+\left[L_{n}, \alpha_{k}\right]\left(f_{k}^{(2)}-i f_{k}^{(1)}\right)\right) \\
& =i \alpha_{n} \cdot f_{0}^{(2)}+\frac{1}{\sqrt{2}} \sum_{k=1}^{\infty} k\left(\alpha_{n+k} \cdot\left(f_{k}^{(2)}-i f_{k}^{(1)}\right)-\alpha_{n-k} \cdot\left(f_{k}^{(2)}+i f_{k}^{(1)}\right)\right)
\end{aligned}
$$

For this to make sense on $F_{00}$, we also need to restrict $f$ to be in $S_{0}$. So the infinitesimal time translations are:

$$
\begin{aligned}
\left(\operatorname{ad} i L_{0}\right)(\Phi(f)) & =\left[i L_{0}, \Phi(f)\right] \\
& =-\alpha_{0} \cdot f_{0}^{(2)}+\frac{1}{\sqrt{2}} \sum_{k=1}^{\infty} k\left(\alpha_{k} \cdot\left(f_{k}^{(1)}+i f_{k}^{(2)}\right)+\alpha_{-k}\left(f_{k}^{(1)}-i f_{k}^{(2)}\right)\right) \\
& =-2 \alpha^{\prime} p \cdot f_{0}^{(2)}+\sum_{k=1}^{\infty} k\left(p_{k} \cdot\left(-f_{k}^{(2)}\right)+q_{k} \cdot f_{k}^{(1)}\right) \\
& =: \Phi\left(T_{0} f\right) \quad \forall f \in S_{0},
\end{aligned}
$$

where $\quad\left(T_{0} f\right)^{(1)}:=\left(-2 \alpha^{\prime} f_{0}^{(2)},-f_{1}^{(1)},-2 f_{2}^{(2)},-3 f_{3}^{(2)}, \ldots\right)$,

$$
\left(T_{0} f\right)^{(2)}:=\left(0, f_{1}^{(1)}, 2 f_{2}^{(1)}, 3 f_{3}^{(1)}, \ldots\right) \quad \forall f \in S_{0} \text {. }
$$

Observe that $T_{0}$ preserves $S_{0}$, though it does not preserve $S$. Next we need to find the infinitesimal gauge transformations ad $i N_{n}$ and ad $i M_{n}$ with $N_{n}:=L_{n}+L_{-n}$, $M_{n}:=i\left(L_{n}-L_{-n}\right)$,

$$
\begin{aligned}
{\left[L_{-n}, \Phi(f)\right]=} & -\left(\left[L_{n}, \Phi(f)\right]\right)^{+} \\
= & i \alpha_{-n} \cdot f_{0}^{(2)}+\frac{1}{\sqrt{2}} \sum_{k=1}^{\infty} k\left(\alpha_{-n-k} \cdot\left(-f_{k}^{(2)}-i f_{k}^{(1)}\right)\right. \\
& \left.\quad+\alpha_{k-n} \cdot\left(f_{k}^{(2)}-i f_{k}^{(1)}\right)\right), \\
{\left[N_{n}, \Phi(f)\right]=} & {\left[L_{n}+L_{-n}, \Phi(f)\right] } \\
= & i\left(\alpha_{n}+\alpha_{-n}\right) \cdot f_{0}^{(2)}+\frac{1}{\sqrt{2}} \sum_{k=1}^{\infty} k\left[\left(f_{k}^{(2)}-i f_{k}^{(1)}\right) \cdot\left(\alpha_{k-n}+\alpha_{k+n}\right)\right. \\
& \left.\quad-\left(f_{k}^{(2)}+i f_{k}^{(1)}\right) \cdot\left(\alpha_{n-k}+\alpha_{-n-k}\right)\right]
\end{aligned}
$$




$$
\begin{aligned}
& =i \sqrt{2} q_{n} \cdot f_{0}^{(2)}+\sum_{k=1}^{\infty} \frac{k}{\sqrt{2}}\left[f_{k}^{(2)} \cdot\left(\left(\alpha_{k-n}-\alpha_{n-k}\right)+\left(\alpha_{k+n}-\alpha_{-k-n}\right)\right)\right. \\
& \left.-i f_{k}^{(1)} \cdot\left(\left(\alpha_{k-n}+\alpha_{n-k}\right)+\left(\alpha_{k+n}+\alpha_{-n-k}\right)\right)\right] \\
& =i \sqrt{2} q_{n} \cdot f_{0}^{(2)}-i \sum_{k=1}^{\infty} k\left(f_{k}^{(2)} \cdot p_{|n-k|} \operatorname{sign}(n-k)-f_{k}^{(2)} \cdot p_{k+n}\right. \\
& \left.+f_{k}^{(1)} \cdot q_{n+k}+f_{k}^{(1)} \cdot q_{|n-k|}\right) \\
& =i \sqrt{2} q_{n} \cdot f_{0}^{(2)}-i \sum_{k=1}^{\infty} k\left(f_{k}^{(1)} \cdot q_{n+k}-f_{k}^{(2)} \cdot p_{n+k}\right)-i \sqrt{2} n f_{n}^{(1)} \cdot \alpha_{0} \\
& -i \sum_{k=1}^{n-1} k\left(f_{k}^{(2)} \cdot p_{n-k}+f_{k}^{(1)} \cdot q_{n-k}\right) \\
& -i \sum_{k=n+1}^{\infty} k\left(f_{k}^{(1)} \cdot q_{k-n}-f_{k}^{(2)} \cdot p_{k-n}\right) \\
& =i \sqrt{2} q_{n} \cdot f_{0}^{(2)}-i \sqrt{2} n f_{n}^{(1)} \cdot 2 \alpha^{\prime} p \\
& -i \sum_{k=n+1}^{\infty}(k-n)\left(f_{k-n}^{(1)} \cdot q_{k}-f_{k-n}^{(2)} \cdot p_{k}\right) \\
& -i \sum_{k=1}^{n-1}(n-k)\left(f_{n-k}^{(2)} \cdot p_{k}+f_{n-k}^{(1)} \cdot q_{k}\right) \\
& -i \sum_{k=1}^{n-1}(k+n)\left(f_{k+n}^{(1)} \cdot q_{k}-f_{k+n}^{(2)} \cdot p_{k}\right)-i 2 n\left(f_{2 n}^{(1)} \cdot q_{n}-f_{2 n}^{(2)} \cdot p_{n}\right) \\
& -i \sum_{k=n+1}^{\infty}(n+k)\left(f_{k+n}^{(1)} \cdot q_{k}-f_{k+n}^{(2)} \cdot p_{k}\right) \\
& =-i \sqrt{2} \alpha^{\prime} n f_{n}^{(1)} \cdot p 2-i q_{n} \cdot\left(2 n f_{2 n}^{(1)}-\sqrt{2} f_{0}^{(2)}\right)+i 2 n f_{2 n}^{(2)} \cdot p_{n} \\
& -i \sum_{k=1}^{n-1}\left\{q_{k} \cdot\left((n+k) f_{n+k}^{(1)}+(n-k) f_{n-k}^{(1)}\right)\right. \\
& +p_{k} \cdot\left((n-k) f_{n-k}^{(2)}-(n+k) f_{n+k}^{(2)}\right\} \\
& -i \sum_{k=n+1}^{\infty}\left\{q_{k} \cdot\left((n+k) f_{n+k}^{(1)}+(k-n) f_{k-n}^{(1)}\right)\right. \\
& \left.+p_{k} \cdot\left((n-k) f_{k-n}^{(2)}-(n+k) f_{n+k}^{(2)}\right)\right\} \\
& =:-i \Phi\left(T_{n} f\right),
\end{aligned}
$$


i.e. $\left(\operatorname{ad} i N_{n}\right)(\Phi(f))=\Phi\left(T_{n} f\right) \forall f \in S$, where:

$$
\begin{aligned}
& \left(T_{n} f\right)_{0}^{(1)}=2 \sqrt{2} \alpha^{\prime} n f_{n}^{(1)}, \\
& \left(T_{n} f\right)_{0}^{(2)}=0, \\
& \left(T_{n} f\right)_{n}^{(1)}=-2 n f_{2 n}^{(2)}, \\
& \left(T_{n} f\right)_{n}^{(2)}=2 n f_{2 n}^{(1)}-\sqrt{2} f_{0}^{(2)}, \\
& \left(T_{n} f\right)_{k}^{(1)}=(n-k) f_{|n-k|}^{(2)}-(n+k) f_{n+k}^{(2)}, \quad k \neq n, \\
& \left(T_{n} f\right)_{k}^{(2)}=|n-k| f_{|n-k|}^{(2)}+(n+k) f_{n+k}^{(1)}, \quad k \neq n .
\end{aligned}
$$

By an entirely similar calculation we find $\left(\operatorname{ad} i M_{n}\right)(\Phi(f))=\Phi\left(R_{n} f\right)$, where

$$
\begin{aligned}
& \left(R_{n} f\right)_{0}^{(1)}=2 \sqrt{2} \alpha^{\prime} f_{n}^{(2)} n, \\
& \left(R_{n} f\right)_{0}^{(2)}=0, \\
& \left(R_{n} f\right)_{k}^{(1)}=(n+k) f_{n+k}^{(1)}-|n-k| f_{|n-k|}^{(1)}+\sqrt{2} f_{0}^{(2)} \delta_{k, n}, \quad k \geqq 1, \\
& \left(R_{n} f\right)_{k}^{(2)}=(n+k) f_{n+k}^{(2)}+(n-k) f_{|n-k|}^{(2)}, \quad k \geqq 1 .
\end{aligned}
$$

Observe that $T_{n}$ and $R_{n}$ preserve $S_{0}$ though not $S$. It is possible to show by direct calculation that $T_{n}$ and $R_{n}$ are infinitesimally symplectic operators on $S_{0}$ (and this provides a useful check on the somewhat cumbersome algebra), but it is far easier to deduce it from the consistency of the setup and:

$$
\begin{aligned}
B\left(T_{n} f, h\right)+B\left(f, T_{n} h\right) & =-i\left(\left[\Phi\left(T_{n} f\right), \Phi(h)\right]+\left[\Phi(f), \Phi\left(T_{n} h\right)\right]\right) \\
& =i\left(\left[\left[i N_{n}, \Phi(f)\right], \Phi(h)\right]+\left[\Phi(f),\left[i N_{n}, \Phi(h)\right]\right]=0,\right.
\end{aligned}
$$

making use of the Jacobi identity and $\left[i N_{n},[\Phi(f), \Phi(h)]\right]=0$.

Lemma A.5. Given for $n \geqq 1, \tilde{L}_{n}:=\frac{1}{2 \alpha^{\prime}} \sum_{k=1}^{\infty} \alpha_{-k} \cdot \alpha_{n+k}, \dot{L}_{n}:=L_{n}-\tilde{L}_{n}$, with the corresponding $\dot{N}_{n}, \dot{M}_{n}, \tilde{N}_{n}, \tilde{M}_{n}$ operators as in Sect. 5. Then

$$
\left[i \tilde{N}_{n}, \Phi(f)\right] \chi=\Phi\left(\tilde{T}_{n} f\right) \chi
$$

and

$$
\left[i \tilde{M}_{n}, \Phi(f)\right] \chi=\Phi\left(\tilde{R}_{n} f\right) \chi \quad \forall f \in S_{0}, \chi \in F_{00}
$$

with $\tilde{T}_{n}, \tilde{R}_{n}$ as in Sect. 5.

Proof. First observe that

$$
\left[\tilde{L_{n}}, \alpha_{m}^{\mu}\right]=\frac{1}{2 \alpha^{\prime}} \sum_{k=1}^{\infty}\left[\alpha_{-k} \cdot \alpha_{n+k}, \alpha_{m}^{\mu}\right]= \begin{cases}0 & \text { if }-n \leqq m<0, \\ m \alpha_{n+m}^{\mu} & \text { otherwise }\end{cases}
$$


$\left[\tilde{L}_{-n}, \alpha_{m}^{\mu}\right]=0$ if $1 \leqq m \leqq n$ and $m \alpha_{-n+m}^{\mu}$ otherwise. So:

$$
\begin{aligned}
& {\left[\tilde{N}_{n}, \alpha_{m}^{\mu}\right]=\left[\tilde{L}_{n}+\tilde{L}_{-n}, \alpha_{m}^{\mu}\right]= \begin{cases}m \alpha_{n+m}^{\mu} & \text { if } 0 \leqq m \leqq n \\
m \alpha_{m-n}^{\mu} & \text { if }-n \leqq m \leqq 0, \\
m\left(\alpha_{n+m}^{\mu}+\alpha_{m-n}^{\mu}\right) & \text { otherwise },\end{cases} } \\
& {\left[\tilde{M}_{n}, \alpha_{m}^{\mu}\right]=i\left[\tilde{L}_{n}-\tilde{L}_{-n}, \alpha_{m}^{\mu}\right]= \begin{cases}i m \alpha_{n+m}^{\mu} & \text { if } 0 \leqq m \leqq n, \\
-i m \alpha_{m-n}^{\mu} & \text { if }-n \leqq m \leqq 0, \\
i m\left(\alpha_{n+m}^{\mu}-\alpha_{m-n}^{\mu}\right) & \text { otherwise } .\end{cases} }
\end{aligned}
$$

So using the smearing formula:

$$
\Phi(f)=p \cdot f_{0}^{(1)}+q \cdot f_{0}^{(2)}+\frac{1}{\sqrt{2}} \sum_{k=1}^{\infty}\left(i\left(\alpha_{-k}-\alpha_{k}\right) \cdot f_{k}^{(1)}+\left(\alpha_{-k}+\alpha_{k}\right) \cdot f_{k}^{(2)}\right),
$$

we obtain:

$$
\begin{aligned}
{\left[\tilde{N}_{n}, \Phi(f)\right]=} & \frac{1}{\sqrt{2}} \sum_{k=1}^{\infty}\left(\left[\tilde{N}_{n}, \alpha_{-k}\right] \cdot\left(f_{k}^{(2)}+i f_{k}^{(1)}\right)+\left[\tilde{N}_{n}, \alpha_{k}\right] \cdot\left(f_{k}^{(2)}-i f_{k}^{(1)}\right)\right) \\
= & \frac{1}{\sqrt{2}} \sum_{k=1}^{n} k\left\{\left(\alpha_{n+k}-\alpha_{-n-k}\right) \cdot f_{k}^{(2)}-i\left(\alpha_{n+k}+\alpha_{-n-k}\right) \cdot f_{k}^{(1)}\right\} \\
& -\frac{1}{\sqrt{2}} \sum_{k=n+1}^{\infty} k\left\{\left(\alpha_{n-k}-\alpha_{k-n}\right) \cdot f_{k}^{(2)}+i\left(\alpha_{n-k}+\alpha_{k-n}\right) \cdot f_{k}^{(1)}\right. \\
& \left.+\left(\alpha_{-k-n}-\alpha_{n+k}\right) \cdot f_{k}^{(2)}+i\left(\alpha_{n+k}+\alpha_{-n-k}\right) \cdot f_{k}^{(1)}\right\} \\
= & \sum_{k=1}^{n} k_{k}\left(p_{n+k} \cdot f_{k}^{(2)}-q_{n+k} \cdot f_{k}^{(1)}\right) \\
& -\sum_{k=n+1}^{\infty} k i\left(-p_{k-n} \cdot f_{k}^{(2)}+q_{k-n} \cdot f_{k}^{(1)}\right. \\
& \left.-p_{n+k} \cdot f_{k}^{(2)}+q_{n+k} \cdot f_{k}^{(1)}\right) \\
= & \left.i \sum_{k=n+1}^{2 n}(k-n)\left(p_{k} \cdot f_{k-n}^{(2)}\right)-q_{k} \cdot f_{k-n}^{(1)}\right) \\
& +i \sum_{k=1}^{\infty}(k+n)\left(p_{k} \cdot f_{k+n}^{(2)}-q_{k} \cdot f_{k+n}^{(1)}\right) \\
& +i \sum_{k=2 n+1}^{\infty}(k-n)\left(p_{k} \cdot f_{k-n}^{(2)}-q_{k} \cdot f_{k-n}^{(1)}\right) \\
= & i \sum_{k=1}^{\infty}(k+n)\left(p_{k} \cdot f_{k+n}^{(2)}-q_{k} \cdot f_{k+n}^{(1)}\right) \\
& +i k-n)\left(p_{k} \cdot f_{k-n}^{(2)}-q_{k} \cdot f_{k-n}^{(1)}\right) \\
& \\
& \\
& \\
& \\
&
\end{aligned}
$$




$$
\begin{aligned}
& =-i \Phi\left(\tilde{T}_{n} f\right), \text { where } \tilde{T}_{n}=\left(\begin{array}{cc}
0 & \tilde{s}_{n} \\
-\tilde{s}_{n} & 0
\end{array}\right), \text { and } \\
& \left(\tilde{s}_{n} f\right)_{k}:=-(n+k) f_{n+k}-(k-n) \theta(k-n) f_{k-n} .
\end{aligned}
$$

From an entirely similar calculation we find $\left[\tilde{M}_{n}, \Phi(f)\right]=-i \Phi\left(\tilde{R}_{n} f\right)$.

Proposition A.6. The exponential series $\sum_{k=0}^{\infty} \frac{\lambda^{k} T_{n}^{k}}{k !} f, n>0$ converges absolutely in the Hilbert norm of $S$ for all $f \in S_{0}$ and for all $\lambda \in\left(-\frac{1}{2 n}, \frac{1}{2 n}\right)$. This series diverges in the Hilbert norm topology for all nonzero $f \in S_{0}$ and $\lambda \in \mathbb{R} \backslash\left(-\frac{1}{n}, \frac{1}{n}\right)$. In the region of convergence, denote the limit by $\exp \left(\lambda T_{n}\right) f, n>0$.

We prepare three lemmata for the proof:

\section{Lemma A.7.}

$$
\begin{aligned}
T_{n}^{2 k} & =\left(\begin{array}{cc}
\left(s_{n} p_{n}\right)^{k} & \left(s_{n} p_{n}\right)^{k-1} s_{n} r_{n}+t_{n} s_{n}\left(p_{n} s_{n}\right)^{k-1} \\
0 & \left(p_{n} s_{n}\right)^{k}
\end{array}\right), \quad k \geqq 1 . \\
T_{n}^{2 k+1} & =\left(\begin{array}{cc}
t_{n}\left(s_{n} p_{n}\right)^{k} & \left(s_{n} p_{n}\right)^{k} s_{n} \\
\left(p_{n} s_{n}\right)^{k} p_{n} & \left(p_{n} s_{n}\right)^{k} r_{n}
\end{array}\right), \quad k \geqq 0 .
\end{aligned}
$$

Proof. Observe from the matrix expressions that:

$$
\begin{aligned}
& t_{n}^{2}=0=r_{n}^{2}, \quad p_{n} t_{n}=0=r_{n} p_{n}, \\
& (2 n+1)^{\mathrm{st}} \\
& \text { column } \\
& t_{n} s_{n}=\left(\begin{array}{cccc}
0 \ldots & 0 & -4 \sqrt{2} \alpha^{\prime} n^{2} & 0 \ldots \\
0 \ldots & 0 & 0 & 0 \ldots \\
\vdots & \vdots & \vdots &
\end{array}\right) \text { and } \\
& s_{n} r_{n}=\underset{(2 n+1) \text { st }}{\operatorname{row}}\left(\begin{array}{ccc}
0 & 0 & \ldots \\
\vdots & & \\
0 & \ldots & \\
\sqrt{2 n} & 0 & \ldots \\
0 & \ldots & \\
\vdots & &
\end{array}\right)
\end{aligned}
$$

Now $p_{n}$ or $s_{n}$ applied to a sequence shifts its entries by $n$ modes, multiplying also by a number. So the first column of $\left(p_{n} s_{n}\right) r_{n}$ is $2 \sqrt{2} n^{2}(0, \ldots, 0,1,0, \ldots$, $0,1,0, \ldots)^{T}$ and it is zero elsewhere, and the nonzero entries are in the rows labelled $(n+1)$ and $(3 n+1)$. In general $\left(p_{n} s_{n}\right)^{k} r_{n}$ has nonzero entries only in the first column in the rows labelled $(m n+1)$, where $m$ must be odd. Hence from the 
form of $t_{n} s_{n}$ displayed above, we see:

$$
t_{n} s_{n}\left(p_{n} s_{n}\right)^{k} r_{n}=0=t_{n}\left(s_{n} p_{n}\right)^{k} s_{n} r_{n} \quad \forall k \geqq 0 .
$$

To prove (i) we do induction on $k$. If $k=1$, by the equations above:

$$
\begin{aligned}
T_{n}^{2}=\left(\begin{array}{cc}
t_{n} & s_{n} \\
p_{n} & r_{n}
\end{array}\right)^{2} & =\left(\begin{array}{cc}
t_{n}^{2}+s_{n} p_{n} & t_{n} s_{n}+s_{n} r_{n} \\
p_{n} t_{n}+r_{n} p_{n} & p_{n} s_{n}+r_{n}^{2}
\end{array}\right) \\
& =\left(\begin{array}{cc}
s_{n} p_{n} & t_{n} s_{n}+s_{n} r_{n} \\
0 & p_{n} s_{n}
\end{array}\right) .
\end{aligned}
$$

Now assume that (i) holds for $k$ and prove it for $k+1$ :

$$
\begin{aligned}
T_{n}^{2(k+1)}= & T_{n}^{2 k} T_{n}^{2}=\left(\begin{array}{cc}
\left(s_{n} p_{n}\right)^{k} & \left(s_{n} p_{n}\right)^{k-1} s_{n} r_{n}+t_{n} s_{n}\left(p_{n} s_{n}\right)^{k-1} \\
0 & \left(p_{n} s_{n}\right)^{k}
\end{array}\right) \\
& \times\left(\begin{array}{cc}
s_{n} p_{n} & t_{n} s_{n}+s_{n} r_{n} \\
0 & p_{n} s_{n}
\end{array}\right) \\
= & \left(\begin{array}{cc}
\left(s_{n} p_{n}\right)^{k+1}\left(s_{n} p_{n}\right)^{k} s_{n} r_{n}+t_{n} s_{n}\left(p_{n} s_{n}\right)^{k} \\
0 & \left(p_{n} s_{n}\right)^{k+1}
\end{array}\right)
\end{aligned}
$$

using the identities above, which completes the induction.
To prove (ii) we also do induction on $k$. When $k=0, T_{n}=\left(\begin{array}{ll}t_{n} & s_{n} \\ p_{n} & r_{n}\end{array}\right)$ as required.
Assume that (ii) holds for $k$ and prove it for $k+1$ :

$$
\begin{aligned}
T_{n}^{2(k+1)+1} & =T_{n}^{2 k+1} T_{n}^{2}=\left(\begin{array}{cc}
t_{n}\left(s_{n} p_{n}\right)^{k} & \left(s_{n} p_{n}\right)^{k} s_{n} \\
\left(p_{n} s_{n}\right)^{k} p_{n} & \left(p_{n} s_{n}\right)^{k} r_{n}
\end{array}\right)\left(\begin{array}{cc}
s_{n} p_{n} & t_{n} s_{n}+s_{n} r_{n} \\
0 & p_{n} s_{n}
\end{array}\right) \\
& =\left(\begin{array}{ll}
t_{n}\left(s_{n} p_{n}\right)^{k+1} & \left(s_{n} p_{n}\right)^{k+1} s_{n} \\
\left(p_{n} s_{n}\right)^{k+1} p_{n} & \left(p_{n} s_{n}\right)^{k+1} r_{n}
\end{array}\right),
\end{aligned}
$$

which completes the induction

Lemma A.8. Using our previous notation where $\varepsilon_{n}$ is the unit vector for the $n^{\text {th }}$ mode in $l_{0}$, we have the following estimates where $k \geqq 1$ :

$$
\begin{aligned}
\left\|\left(s_{n}\right)^{k} \varepsilon_{m}\right\| & \leqq \sqrt{\alpha^{\prime}} 2^{k+1} \prod_{l=0}^{k}(m+l n) \geqq\left\|\left(p_{n}\right)^{k} \varepsilon_{m}\right\|, \\
\left\|\left(s_{n} p_{n}\right)^{k} \varepsilon_{m}\right\| & \leqq \sqrt{\alpha^{\prime}} 2^{2 k+1} \prod_{l=0}^{2 k}(m+l n) \geqq\left\|\left(p_{n} s_{n}\right)^{k} \varepsilon_{m}\right\| \\
\left\|t_{n} s_{n}\left(p_{n} s_{n}\right)^{k-1} \varepsilon_{m}\right\| & \leqq n^{2} \alpha^{\prime} 2^{2 k+1} \prod_{l=0}^{2 k-1}(m+l n), \\
\left\|\left(s_{n} p_{n}\right)^{k-1} s_{n} r_{n} \varepsilon_{m}\right\| & \leqq \delta_{0, m} \sqrt{2 \alpha^{\prime}}(2 n)^{2 k}(2 k) !, \text { and } s_{n} \varepsilon_{0}=0=p_{n} \varepsilon_{0} .
\end{aligned}
$$

Proof. The last equation follows directly from definitions. Recall that the norm in $\tilde{l}^{2}$ is $\|f\|^{2}=f_{0}^{2}+2 \alpha^{\prime} \sum_{m=1}^{\infty} m f_{m}^{2}$, and so $\left\|\varepsilon_{m}\right\|^{2}=\delta_{0, m}+2 \alpha^{\prime} m\left(1-\delta_{0, m}\right)$ (and so in general for $f=\sum_{m=0}^{M} \lambda_{m} \varepsilon_{m} \in l_{0}$, we find $\left.\|f\|^{2}=\lambda_{0}^{2}+2 \alpha^{\prime} \sum_{m=1}^{M} m \lambda_{m}^{2}\right)$. By 
definitions:

$$
\begin{aligned}
& s_{n} \varepsilon_{m}= \begin{cases}m\left(\varepsilon_{n-m}-\varepsilon_{n+m}\right), & \text { if } m<n ; \\
-n \varepsilon_{2 n}, & \text { if } m=n ; \\
-m\left(\varepsilon_{m-n}+\varepsilon_{m+n}\right) ; & \text { if } m>n,\end{cases} \\
& \text { so: }\left\|s_{n} \varepsilon_{m}\right\|^{2} \leqq m^{2}\left\|\varepsilon_{n-m}\right\|^{2}+m^{2}\left\|\varepsilon_{n+m}\right\|^{2}=4 \alpha^{\prime} m^{2} n \\
& \leqq 2 m^{2}\left\|\varepsilon_{n+m}\right\|^{2}=4 \alpha^{\prime} m^{2}(n+m) .
\end{aligned}
$$

The same inequality is true for $p_{n} \varepsilon_{m}$ because $s_{n}$ and $p_{n}$ only differ in the signs of the coefficients. Observe now that $s_{n}$ and $p_{n}$ applied to an $\varepsilon_{m}$ create at most two $\varepsilon_{i}$ 's, multiplied by $\pm m$, and so for a monomial in $s_{n}$ and $p_{n}$ applied to $\varepsilon_{m}$, the magnitude of its leading term (i.e. the entry for the highest mode which is nonzero) is always larger than all the other stepwise created terms. That is,

$$
\begin{aligned}
\left\|\left(s_{n}\right)^{k} \varepsilon_{m}\right\| & \leqq 2^{k}\left\|L\left(\left(s_{n}\right)^{k} \varepsilon_{m}\right)\right\|, \quad\left\|\left(p_{n}\right)^{k} \varepsilon_{m}\right\| \leqq 2^{k}\left\|L\left(\left(p_{n}\right)^{k} \varepsilon_{m}\right)\right\|, \\
\left\|\left(s_{n} p_{n}\right)^{k} \varepsilon_{m}\right\| & \leqq 2^{2 k}\left\|L\left(\left(s_{n} p_{n}\right)^{k} \varepsilon_{m}\right)\right\|, \quad \text { etc. },
\end{aligned}
$$

where $L(f)$ for $f \in l_{0}$ denotes the leading term of $f$. By examination of the forms of $s_{n}$ and $p_{n}$ we see:

$$
L\left(\left(s_{n}\right)^{k} \varepsilon_{m}\right)= \pm \varepsilon_{m+k n} \sum_{l=0}^{k-1}(m+\ln )=L\left(\left(p_{n}\right)^{k} \varepsilon_{m}\right), \text { etc. }
$$

Hence

$$
\begin{aligned}
\left\|\left(s_{n}\right)^{k} \varepsilon_{m}\right\|^{2} & \leqq 2 \alpha^{\prime}(m+k n) 2^{2 k} \prod_{l=0}^{k-1}(m+l n)^{2} \\
& \leqq \alpha^{\prime} 2^{2 k+2} \prod_{l=0}^{k}(m+\ln )^{2}
\end{aligned}
$$

i.e.

$$
\left\|\left(s_{n}\right)^{k} \varepsilon_{m}\right\| \leqq \sqrt{\alpha^{\prime}} 2^{k+1} \prod_{l=0}^{k}(m+\ln ),
$$

and exactly the same inequality holds for any monomial of order $k$ in $s_{n}$ and $p_{n}$. For the next inequality of A.8, observe from the form of $t_{n} s_{n}$ at the start of the proof of A.7, that $t_{n} s_{n} f$ picks out the $2 n^{\text {th }}$ mode of $f$, multiply by $-4 \sqrt{2} \alpha^{\prime} n^{2}$, and insert this into the $0^{\text {th }}$ mode (whose norm differs from that of the other modes by a factor of $\left.\left(2 \alpha^{\prime}\right)^{1 / 2}\right)$. So since $\left\|f_{2 n}\right\| \leqq\|f\|$,

$$
\begin{aligned}
\left\|t_{n} s_{n}\left(p_{n} s_{n}\right)^{k-1} \varepsilon_{m}\right\| & \leqq \frac{4 \sqrt{2} \alpha^{\prime} n^{2}}{\left(2 \alpha^{\prime}\right)^{1 / 2}}\left\|\left(p_{n} s_{n}\right)^{k-1} \varepsilon_{m}\right\| \\
& \leqq n^{2} \alpha^{\prime} 2^{2 k+1} \prod_{l=0}^{2 k-2}(m+l n), \\
\left\|\left(s_{n} p_{n}\right)^{k-1} s_{n} r_{n} \varepsilon_{0}\right\| & =\left\|\left(s_{n} p_{n}\right)^{k-1} \varepsilon_{n} \sqrt{2}\right\| \\
& \leqq \sqrt{2 \alpha^{\prime}} 2^{2 k} \prod_{l=0}^{2 k-1}(1+l) n=\sqrt{2 \alpha^{\prime}}(2 n)^{2 k}(2 k) !
\end{aligned}
$$

and $r_{n} \varepsilon_{m}=0$ for $m \neq 0$. 
Lemma A.9. Let $\Lambda$ be any monomial of order $k$ in $s_{n}$ and $p_{n}$. Then

$$
\left\|\Lambda \varepsilon_{m}\right\| \geqq \sqrt{2 \alpha^{\prime}(m+k n)} \prod_{l=0}^{k-1}(m+l n) .
$$

Proof. Due to orthogonality considerations, $\|f\| \geqq\|L(f)\|$ for all $f \in l_{0}$, hence since $L\left(\Lambda \varepsilon_{m}\right)= \pm \varepsilon_{m+k n} \prod_{l=0}^{k-1}(m+l n)$, we obtain $\left\|\Lambda \varepsilon_{m}\right\|^{2} \geqq$ $2 \alpha^{\prime}(m+k n)\left(\prod_{l=0}^{k-1}(m+\ln )\right)^{2}$.

Proof of A.6. We first do the convergence of $\sum_{k=0}^{\infty} \frac{\lambda^{k} T_{n}^{k}}{k !} f$ for $f \in S_{0}$. Since $T_{n}$ acts as the identity on $M^{D+1}$, i.e. it does not mix space-time indices and acts identically for each, it suffices to show convergence with respect to the Hilbert space topology of $\mathbb{R}^{2} \otimes \tilde{l}^{2}$. Observe now that $\left\{\delta_{m}^{(1)}:=\left(\begin{array}{l}1 \\ 0\end{array}\right) \otimes \varepsilon_{m}, \delta_{m}^{(2)}:=\left(\begin{array}{l}0 \\ 1\end{array}\right) \otimes \varepsilon_{m} ; m \in \mathbb{Z}_{+}\right\}$is a real basis for $\mathbb{R}^{2} \otimes l_{0}$ and that $T_{n}$ is real linear. So for $f=\sum_{j=1}^{2} \sum_{m=0}^{M_{j}} \lambda_{m}^{(j)} \delta_{m}^{(j)}$, $\lambda_{m}^{(j)} \in \mathbb{R}$, we get $\sum_{k=0}^{N} \frac{\lambda^{k} T_{n}^{k}}{k !} f=\sum_{j=1}^{2} \sum_{m=0}^{M_{j}} \lambda_{m}^{(j)} \sum_{k=0}^{N} \frac{\lambda^{k} T_{n}^{k}}{k !} \delta_{m}^{(j)}$. Hence it suffices to show that $\sum_{k=0}^{N} \frac{\lambda^{k} T_{n}^{k}}{k !} \delta_{m}^{(j)}$ converges for the Hilbert space topology of $\mathbb{R}^{2} \otimes \tilde{l}^{2}$. Using A.7 write for $N$ even:

$$
\begin{aligned}
\sum_{k=0}^{N} \frac{\lambda^{k} T_{n}^{k}}{k !}=1 & +\sum_{k=1}^{N / 2} \frac{\lambda^{2 k} T_{n}^{2 k}}{(2 k) !}+\sum_{k=0}^{N / 2-1} \frac{\lambda^{2 k+1} T_{n}^{2 k+1}}{(2 k+1) !} \\
=1 & +\sum_{k=1}^{N / 2} \frac{\lambda^{2 k}}{(2 k) !}\left(\begin{array}{cc}
\left(s_{n} p_{n}\right)^{k} & \left(s_{n} p_{n}\right)^{k-1} s_{n} r_{n}+t_{n} s_{n}\left(p_{n} s_{n}\right)^{k-1} \\
0 & \left(p_{n} s_{n}\right)^{k}
\end{array}\right) \\
& +\sum_{k=0}^{N / 2-1} \frac{\lambda^{2 k+1}}{(2 k+1) !}\left(\begin{array}{cc}
t_{n}\left(s_{n} p_{n}\right)^{k} & \left(s_{n} p_{n}\right)^{k} s_{n} \\
\left(p_{n} s_{n}\right)^{k} p_{n} & \left(p_{n} s_{n}\right)^{k} r_{n}
\end{array}\right),
\end{aligned}
$$

and it suffices to show that both the even and odd series converge on $\delta_{m}^{(j)}$ as $N \rightarrow \infty$. From A.7 and the norm of $\mathbb{R}^{2} \otimes \tilde{l}^{2}$ we see:

$$
\begin{aligned}
\left\|T_{n}^{2 k} \delta_{m}^{(1)}\right\| & =\left\|\left(s_{n} p_{n}\right)^{k} \varepsilon_{m}\right\|, \\
\left\|T_{n}^{2 k} \delta_{m}^{(2)}\right\|^{2} & =\left\|\left(s_{n} p_{n}\right)^{k-1} s_{n} r_{n} \varepsilon_{m}+t_{n} s_{n}\left(p_{n} s_{n}\right)^{k-1} \varepsilon_{m}\right\|^{2}+\left\|\left(p_{n} s_{n}\right)^{k} \varepsilon_{m}\right\|^{2}, \\
\left\|T_{n}^{2 k+1} \delta_{m}^{(1)}\right\|^{2} & =\left\|t_{n}\left(s_{n} p_{n}\right)^{k} \varepsilon_{m}\right\|^{2}+\left\|\left(p_{n} s_{n}\right)^{k} p_{n} \varepsilon_{m}\right\|^{2}, \\
\left\|T_{n}^{2 k+1} \delta_{m}^{(2)}\right\|^{2} & =\left\|\left(s_{n} p_{n}\right)^{k} s_{n} \varepsilon_{m}\right\|^{2}+\left\|\left(p_{n} s_{n}\right)^{k} r_{n} \varepsilon_{m}\right\|^{2},
\end{aligned}
$$

where the norm on the left-hand sides is that of $\mathbb{R}^{2} \otimes \tilde{l}^{2}$, and the norm on the right-hand sides is that of $\tilde{l}^{2}$. Hence on employment of A.8 we get:

$$
\left\|T_{n}^{2 k} \delta_{m}^{(1)}\right\|=\sqrt{\alpha^{\prime}} 2^{2 k+1} \prod_{l=0}^{2 k}(m+l n), \quad k \geqq 1,
$$


and using orthogonality:

$$
\begin{aligned}
\left\|T_{n}^{2 k} \delta_{m}^{(2)}\right\|^{2}= & \left\|\left(s_{n} p_{n}\right)^{k-1} s_{n} r_{n} \varepsilon_{m}\right\|^{2}+\left\|t_{n} s_{n}\left(p_{n} s_{n}\right)^{k-1} \varepsilon_{m}\right\|^{2}+\left\|\left(p_{n} s_{n}\right)^{k} \varepsilon_{m}\right\|^{2} \\
\leqq & \delta_{0, m} 2 \alpha^{\prime}(2 n)^{4 k}[(2 k) !]^{2}+\left(\alpha^{\prime}\right)^{2} n^{4} 2^{4 k+2} \prod_{l=0}^{2 k-1}(m+l n)^{2} \\
& +\alpha^{\prime} 2^{4 k+2} \prod_{l=0}^{2 k}(m+l n)^{2} \\
\leqq & \delta_{0, m} 2 \alpha^{\prime}(2 n)^{4 k}[(2 k) !]^{2}+\alpha^{\prime}\left(\alpha^{\prime} n^{4}+1\right) 2^{4 k+2} \prod_{l=0}^{2 k}(m+l n)^{2} .
\end{aligned}
$$

Hence

$$
\begin{aligned}
\left\|T_{n}^{2 k} \delta_{m}^{(2)}\right\| & \leqq \delta_{0, m} \sqrt{2 \alpha^{\prime}}(2 n)^{2 k}(2 k) !+\left(\sqrt{\alpha^{\prime}}+\alpha^{\prime} n^{2}\right) 2^{2 k+1} \prod_{l=0}^{2 k}(m+l n), \\
\left\|T_{n}^{2 k+1} \delta_{m}^{(1)}\right\| & \leqq\left\|t_{n}\left(s_{n} p_{n}\right)^{k} \varepsilon_{m}\right\|+\left\|\left(p_{n} s_{n}\right)^{k} p_{n} \varepsilon_{m}\right\| \\
& \leqq \frac{4 \sqrt{2} \alpha^{\prime} n^{2}}{\left(2 \alpha^{\prime}\right)^{1 / 2}}\left\|\left(p_{n} s_{n}^{k-1}\right) p_{n} \varepsilon_{m}\right\|+\sqrt{\alpha^{\prime}} 2^{2 k+2} \prod_{l=0}^{2 k+1}(m+\ln ) \\
& \leqq 4 \alpha^{\prime} n^{2} 2^{2 k} \prod_{l=0}^{2 k-1}(m+\ln )+\sqrt{\alpha^{\prime}} 2^{2 k+2} \prod_{l=0}^{2 k+1}(m+\ln ) \\
& \leqq \sqrt{\alpha^{\prime}}\left(n^{2} \sqrt{\alpha^{\prime}}+1\right) 2^{2 k+2} \prod_{l=0}^{2 k+1}(m+\ln ), \\
\left\|T_{n}^{2 k+1} \delta_{m}^{(2)}\right\| & \leqq \sqrt{\alpha^{\prime}} 2^{2 k+2} \prod_{l=0}^{2 k+1}(m+\ln )+\left\|\left(p_{n} s_{n}\right)^{k} \varepsilon_{n} \sqrt{2}\right\| \delta_{0, m} \\
& \leqq \sqrt{\alpha^{\prime}} 2^{2 k+2} \prod_{l=0}^{2 k+1}(m+\ln )+\sqrt{2 \alpha^{\prime}} 2^{2 k+1} \delta_{0, m} \prod_{l=0}^{2 k}(1+l) n \\
& =\sqrt{\alpha^{\prime}} 2^{2 k+2} \prod_{l=0}^{2 k+1}(m+\ln )+\sqrt{2 \alpha^{\prime}}(2 n)^{2 k+1}(2 k+1) ! \delta_{0, m} .
\end{aligned}
$$

Now return to the series:

$$
\begin{aligned}
\left\|\sum_{k=1}^{N} \frac{\lambda^{2 k} T_{n}^{2 k}}{(2 k) !} \delta_{m}^{(1)}\right\| & \leqq \sum_{k=1}^{N} \frac{\lambda^{2 k}}{(2 k) !}\left\|T_{n}^{2 k} \delta_{m}^{(1)}\right\| \\
& \leqq 2 \sqrt{\alpha^{\prime}} \sum_{k=1}^{N} \frac{(2 \lambda)^{2 k}}{(2 k) !} \prod_{l=0}^{2 k}(m+\ln ) \equiv 2 \sqrt{\alpha^{\prime}} \sum_{k=1}^{N} b_{k},
\end{aligned}
$$

and this will converge as $N \rightarrow \infty$ if it satisfies the ratio test: $\left|\frac{b_{k+1}}{b_{k}}\right| \frac{k}{\infty} t<1$ :

$$
\begin{aligned}
\left|\frac{b_{k+1}}{b_{k}}\right| & =\frac{(2 \lambda)^{2 k+2}}{(2 k+2) !} \cdot \frac{\prod_{l=0}^{2 k+2}(m+\ln )}{\prod_{l=0}^{2 k}(m+\ln )} \cdot \frac{(2 k) !}{(2 \lambda)^{2 k}} \\
& =\frac{(2 \lambda)^{2}(m+2(k+1) n)(m+(2 k+1) n)}{(2 k+2)(2 k+1)} \underset{\infty}{\stackrel{k}{\longrightarrow}(2 \lambda n)^{2},}
\end{aligned}
$$


i.e. this series converges absolutely when $|\lambda|<1 / 2 n$,

$$
\left\|\sum_{k=1}^{N} \frac{\lambda^{2 k} T_{n}^{2 k}}{(2 k) !} \delta_{0}^{(2)}\right\| \leqq \sum_{k=1}^{N} \frac{\lambda^{2 k}}{(2 k) !} \sqrt{2 \alpha^{\prime}}(2 n)^{2 k}(2 k) !=\sqrt{2 \alpha^{\prime}} \sum_{k=1}^{N}(2 \lambda n)^{2 k},
$$

which converges absolutely for $|\lambda|<1 / 2 n$. Now let $m \neq 0$ :

$$
\begin{aligned}
\left\|\sum_{k=1}^{N} \frac{\lambda^{2 k} T_{n}^{2 k}}{(2 k) !} \delta_{m}^{(2)}\right\| & \leqq\left(\sqrt{\alpha^{\prime}}+\alpha^{\prime} n^{2}\right) 2 \sum_{k=1}^{N} \frac{(2 \lambda)^{2 k}}{(2 k) !} \prod_{l=0}^{2 k}(m+l n) \\
& =2\left(\sqrt{\alpha^{\prime}}+\alpha^{\prime} n^{2}\right) \sum_{k=1}^{N} b_{k} .
\end{aligned}
$$

Hence the even series converges absolutely on $S_{0}$ for $|\lambda|<1 / 2 n$. Now examine the odd series:

$$
\begin{aligned}
\left\|\sum_{k=0}^{N} \frac{\lambda^{2 k+1}}{(2 k+1) !} T_{n}^{2 k+1} \delta_{m}^{(1)}\right\| & \leqq 2 \sqrt{\alpha^{\prime}}\left(n^{2} \sqrt{\alpha^{\prime}}+1\right) \sum_{k=1}^{N} \frac{(2|\lambda|)^{2 k+1}}{(2 k+1) !} \prod_{l=0}^{2 k+1}(m+\ln ) \\
& \equiv 2\left(n^{2} \alpha^{\prime}+\sqrt{\alpha^{\prime}}\right) \sum_{k=1}^{N} c_{k},
\end{aligned}
$$

and apply the ratio test as above:

$$
\begin{aligned}
\left|\frac{c_{k+1}}{c_{k}}\right| & =\frac{(2|\lambda|)^{2 k+3}}{(2 k+3) !} \cdot \frac{\prod_{l=0}^{2 k+3}(m+\ln )}{\prod_{l=0}^{2 k+1}(m+\ln )} \cdot \frac{(2 k+1) !}{(2|\lambda|)^{2 k+1}} \\
& =\frac{(2 \lambda)^{2}(m+(2 k+3) n)(m+(2 k+2) n)}{(2 k+3)(2 k+2)} \frac{k}{\infty}(2 \lambda n)^{2},
\end{aligned}
$$

so the odd series on $\delta_{m}^{(1)}$ converges absolutely for $|\lambda|<1 / 2 n$. Now

$$
\left\|\sum_{k=0}^{N} \frac{\lambda^{2 k+1}}{(2 k+1) !} T_{n}^{2 k+1} \delta_{0}^{(2)}\right\| \leqq \sqrt{2 \alpha^{\prime}} \sum_{k=0}^{N} \frac{(2 n|\lambda|)^{2 k+1}}{(2 k+1) !}(2 k+1) !=\sqrt{2 \alpha^{\prime}} \sum_{k=0}^{N}(2 n|\lambda|)^{2 k+1}
$$

with convergence properties as before. Let $m \neq 0$ :

$$
\left\|\sum_{k=0}^{N} \frac{\lambda^{2 k+1}}{(2 k+1) !} T_{n}^{2 k+1} \delta_{m}^{(2)}\right\| \leqq 2 \sqrt{\alpha^{\prime}} \sum_{k=0}^{N} \frac{(2|\lambda|)^{2 k+1}}{(2 k+1) !} \prod_{l=0}^{2 k+1}(m+\ln )=2 \sqrt{\alpha^{\prime}} \sum_{k=0}^{N} c_{k},
$$

which completes the convergence part of the proof of A.6, so $\sum_{k=0}^{\infty} \frac{\lambda^{k} T_{n}^{k}}{k !} f$ converges as required. For the divergence part of the proof we use A.9. By the equations $(*)$ earlier in this proof,

$$
\begin{aligned}
\left\|T_{n}^{2 k} \delta_{m}^{(1)}\right\| & =\left\|\left(s_{n} p_{n}\right)^{k} \varepsilon_{m}\right\|, \quad\left\|T_{n}^{2 k} \delta_{m}^{(2)}\right\| \geqq\left\|\left(p_{n} s_{n}\right)^{k} \varepsilon_{m}\right\|, \\
\left\|T_{n}^{2 k+1} \delta_{m}^{(1)}\right\| & \geqq\left\|\left(p_{n} s_{n}\right)^{k} p_{n} \varepsilon_{m}\right\|, \quad\left\|T_{n}^{2 k+1} \delta_{m}^{(2)}\right\| \geqq\left\|\left(s_{n} p_{n}\right)^{k} s_{n} \varepsilon_{m}\right\|,
\end{aligned}
$$

and so:

$$
\left\|\frac{\lambda^{2 k}}{(2 k) !} T_{n}^{2 k} \delta_{m}^{(1)}\right\| \geqq \sqrt{2 \alpha^{\prime}(m+2 k n)} \frac{\lambda^{2 k}}{(2 k) !} \prod_{l=0}^{2 k+1}(m+\ln ) \equiv \xi_{k} .
$$


Now:

$$
\begin{aligned}
\frac{\xi_{k+1}}{\xi_{k}} & =\frac{\lambda^{2 k+2}}{(2 k+2) !} \cdot \frac{\sqrt{m+(2 k+2) n} \prod_{l=0}^{2 k+3}(m+l n)}{\sqrt{m+2 k n} \prod_{l=0}^{2 k+1}(m+l n)} \cdot \frac{(2 k) !}{\lambda^{2 k}} \\
& =\lambda^{2}\left(\frac{m+(2 k+2) n}{m+2 k n}\right)^{1 / 2} \frac{(m+(2 k+3) n)(m+(2 k+2) n)}{(2 k+2)(2 k+1)} \\
& \underset{\infty}{\stackrel{k}{\longrightarrow}(n \lambda)^{2} .}
\end{aligned}
$$

Hence for $|\lambda|>1 / n, \quad \xi_{k}$ becomes progressively larger with $k$ and hence $\sum_{k=0}^{\infty} \frac{\lambda^{2 k}}{(2 k) !} T_{n}^{2 k} \delta_{m}^{(1)}$ diverges for $|\lambda|>1 / n$. It is sufficient for divergence of a series to prove that it has one subsequence whose terms go to infinity, so we do not consider the other subsequences (which has similar behaviour), and the divergence proof ends here.

Acknowledgements. One of us (H.G.) wishes to thank Prof. Gerry Johnson for mentioning Trotter's formula in a seminar, and also to thank Michael Murray for many discussions on infinite dimensional Lie groups. This project was funded by an ARC grant.

\section{References}

1. Scherck, J.: An introduction to the theory of dual models and strings. Rev. Mod. Phys. 47, 123-163 (1975)

2. Govaerts, J.: String and superstring theories: An introduction. Proceedings of the 2nd Mexican school of particles and fields, Cuernavaca-Morelos, 1986. Eds. Lucio, J.L., Zepeda, A., Singapore: World Scientific 1987, pp. 248-442

3. Pressley, A., Segal, G.: Loop groups. Oxford: Clarendon Press 1986

4. Bognar, J.: Indefinite inner product spaces. Berlin, Heidelberg, New York: Springer 1974

5. Mintchev, M.: Quantization in indefinite metric. J. Phys. A: Math. Gen. 13, 1841-1859 (1980)

6. Pilch, K., Warner, N.P.: Holomorphic structure of superstring vacua. Class. Quantum Grav. 4, 1183-1192 (1987)

7. Araki, H., Shiraishi, M.: On quasifree states of the canonical commutation relations. Publ. RIMS, Kyoto Univ. 7, 105-120 (1971/72)

8. Slawny, J.: On factor representations and the $\mathrm{C}^{*}$-algebra of the canonical commutation relations. Commun. Math. Phys. 24, 151-170 (1972)

9. Manuceau, J.: C*-algebre de relations de commutation. Ann. Inst. Henri Poincaré 8, 139-161 (1968)

10. Emch, G.G.: Algebraic methods in statistical mechanics and quantum field theory. New York: John Wiley 1972

11. Segal, G.: Unitary representations of some infinite dimensional groups. Commun. Math. Phys. 80, 301-342 (1981)

12. Green, M.B., Schwarz, J.H., Witten, E.: Superstring theory volume 1. Cambridge: Cambridge University Press, 1987

13. Mueller, M.: $C^{*}$-algebras and string field theory. Preprint UPR-0367T, Dept. Physics, David Rittenhouse Laboratory, University of Pennsylvania, Philadelphia

14. Buchholz, D., Mack, G., Paunov, R.R., Todorov, I.T.: An algebraic approach to the classification of local conformal quantum field theories. IXth International conference on mathematical physics, Swansea. Eds. Simon, B., Truman, A., Davies, I.M. London: Adam Hilger 1989, pp. 299-305 
15. Mickelsson, J.: String quantization on group manifolds and the holomorphic geometry of Diff $S^{1} / S^{1}$. Commun. Math. Phys. 112, 653-661 (1987)

16. Bowick, M.J., Rajeev, S.G.: String theory as the Kähler geometry of loop space. Phys. Rev. Lett. 58, 535-538 (1987)

17. Bowick, M.J., Rajeev, S.G.: The holomorphic geometry of closed bosonic string theory and Diff $S^{1} / S^{1}$. Nucl. Phys. B293, 348-384 (1987)

18. Alvarez-Gaumé, L., Gomez, C., Reina, C.: Loop groups, Grassmanians and string theory. Phys. Lett B190, 55-62 (1987)

19. Grundling, H., Hurst, C.A.: A note on regular states and supplementary conditions. Lett. Math. Phys. 15, 205-212 (1988), Erratum. Lett. Math. Phys. 17, 173-174 (1989)

20. Glimm, J., Jaffe, A.: Quantum physics. A functional integral point of view. Berlin Heidelberg New York: Springer 1981

21. Nilsson, M.L.: Field equations for the covariantly second quantized bosonic string. Il Nuovo Cim. 101A, 619-629 (1989)

22. Milnor, J.: Remarks on infinite dimensional Lie groups. Relativity, groups and topology II, Les Houches XL, 1983. Eds. DeWitt, B.S., Stora, R., Amsterdam: Elsevier Science 1984, pp. 1009-1057

23. Van Daele, A., Verbeure, A.: Unitary equivalence of Fock representations on the Weyl algebra. Commun. Math. Phys. 20, 268-278 (1971)

24. Jakobczyk, L.: Canonical quantization with indefinite inner product. Ann. Phys. 161, 314-336 (1985)

25. Pedersen, G.K.: C*-algebras and their automorphism groups. London: Academic Press 1979

26. Reed, M., Simon, B.: Methods of modern mathematical physics II. New York: Academic Press 1975

27. Shale, D.: Linear symmetries of free Boson fields. Trans. Am. Math. Soc. 103, 149-167 (1962)

28. Barut, A.O., Raczka, R.: Properties of non-unitary zero mass induced representations of the Poincare group on the space of tensor valued functions. Ann. Inst. H. Poincaré 17, 111-118 (1972)

29. Grundling, H.: Systems with outer constraints. Gupta-Bleuler electromagnetism as an algebraic field theory. Commun. Math. Phys. 114, 69-91 (1988)

30. Packer, J.A., Raeburn, I: Twisted crossed products of $\mathrm{C}^{*}$-algebras. Math. Proc. Camb. Phil. Soc. 106, 293-311 (1989)

31. Sutherland, C.E.: Cohomology and Extensions of von Neumann Algebras I. Publ. RIMS Kyoto Univ. 16, 105-133 (1980)

32. Grundling, H., Hurst, C.A.: Algebraic Quantization of Systems with a Gauge Degeneracy. Commun. Math. Phys. 98, 369-390 (1985)

33. Grundling, H., Hurst, C.A.: The Quantum Thoery of Second Class Constraints: Kinematics. Commun. Math. Phys. 119, 75-93 (1988)

34. Wiesbrock H.-W.: The $C^{*}$-algebra of Bosonic Strings. Commun. Math. Phys. 136, 369-397 (1991)

\section{Addendum}

We have been asked by the referee to compare the string $C^{*}$-algebra constructed here, with the recently published string $C^{*}$-algebra of Wiesbrock [34]. This latter approach draws from a heuristic construction of Witten [35] in which the kinematics of an interacting string is modelled by a groupoid with strings as elements, and where multiplication is joining of overlapping strings. Briefly, Wiesbrock's construction runs as follows. The classical string space consists of the set of continuous paths $\omega:[0,1] \rightarrow \mathbb{R}^{d}$, so classical string theory is given by the algebra of functionals on string space with pointwise multiplication and addition, and the sup norm. In the spirit of noncommutative differential geometry, this system will be quantized by proposing a noncommutative product for these functionals. Each string has a distinguished point at $\frac{1}{2}$ which allows the part $\left[0, \frac{1}{2}\right] \rightarrow \mathbb{R}^{d}$ (resp. $\left[\frac{1}{2}, 1\right] \rightarrow \mathbb{R}^{d}$ ) to be labelled "source" (resp. "range"), and two strings join if the 
range of the first is the source of the second, which leaves the product consisting of the source of the first and the range of the second. For two string functionals $\Phi, \Psi$, Wiesbrock defines the product functional $(\Phi * \Psi)\left(\omega^{s}, \omega^{r}\right)$ as an integral over all possible splittings of $\omega=\left(\omega^{s}, \omega^{r}\right)$, and so need a measure on path space. For this, he chooses a measure $\mu$ consisting of a Gaussian on $\mathscr{S}^{\prime}\left([0,1], \mathbb{R}^{d}\right)$ with covariance operator $\left(-\Delta_{N}^{\prime}\right)^{-1 / 2}$ on $L^{2}[0,1]$ (cf. Glimm and Jaffe [20]), where $-\Delta_{N}^{\prime}$ is the restriction of the Laplacian with Neumann boundary conditions at 0,1 to the complement of its kernel, together with a Lebesgue measure on its kernel. This necessitates the extension of the space of strings from the continuous strings to the Schwartz space $\mathscr{S}^{\prime}\left([0,1], \mathbb{R}^{d}\right)$, in which the set of continuous strings is of $\mu$ measure zero. Moreover

$$
\operatorname{supp} \mu \subset \mathscr{S}^{\prime}\left[0, \frac{1}{2}\right] \times \mathscr{S}^{\prime}\left[\frac{1}{2}, 1\right] \times \mathbb{R},
$$

where for $\omega=\left(\omega^{s}, \omega^{r}, x\right) \in \operatorname{supp} \mu, \omega^{s}$ is the source, $\omega^{r}$ is the range and $x$ is the jump at $\frac{1}{2}$. Given two string functionals $\Phi, \Psi \in(\operatorname{supp} \mu)^{*}$, the product $(\Phi * \Psi)\left(\omega^{s}, \omega^{r}, x\right)$ is defined as convolution over $x$ and an integral over all possible splittings of $\omega=\left(\omega^{s}, \omega^{r}, x\right)$ using the half-string Gaussian $\mu_{r}$ on $\mathscr{S}^{\prime}\left(\left[\frac{1}{2}, 1\right], \mathbb{R}^{d}\right)$ with covariance $\left(-\Delta_{N}^{\prime}\right)^{-1 / 2}$ on $L^{2}\left[\frac{1}{2}, 1\right]$ taking into account Radon-Nikodym derivatives. Involution is naturally defined and this defines a $\mathrm{C}^{*}$-algebra $\mathscr{A}$ as the $\mathrm{C}^{*}$-enveloping algebra of the $L^{1}$-algebra.

Next the dependence on the initial parametrisation must be lifted, so reparametrisations $\gamma \in \operatorname{Diff}[0,1]$ compatible with the groupoid structure (i.e. fixing the points $0, \frac{1}{2}, 1$ ) are taken to comprise the symmetry group Diff ${ }_{W}$ (a Frechet Lie group with Lie algebra found by Witten). However, Diff $_{W}$ does not act as automorphisms on $\mathscr{A}$ because reparametrisation can transform the Gaussian $\mu$ to an incompatible measure (since the covariance $\left(-\Delta_{N}^{\prime}\right)^{1 / 2}$ is not invariant modulo Hilbert-Schmidt under reparametrisation). To deal with this, Wiesbrock uses the Quillen determinant bundle to define a line bundle of which the base is Diff $W_{W}$ and each fibre is identified with a Gaussian measure, reparametrised by the base element in Diff $_{W}$. This bundle needs to be combined with another bundle over Diff ${ }_{W}$ to produce a "Haar measure" on Diff $W$, via a Kahler structure on $M=\left(\text { Diff }^{1} / S^{1}\right)_{0}$ proposed in $[15,16]$, and an identification $\operatorname{Diff}_{W} \subset M$. It starts with a canonical splitting of $T_{1} M$ which produces an almost complex structure on $M$ with respect to the given parametrisation, and making $T_{1} M$ into a Hilbert space $\mathscr{H}$. The resultant complex structure is only invariant modulo Hilbert-Schmidt operators under reparametrisation, which allows identification of $M$ with the index 0 component of the universal Grassmannian over $\mathscr{H}$, which is a Hilbert manifold over the HilbertSchmidt operators. This provides $M$ with a Kahler metric from which one constructs a line bundle over $M$ with Quillen's DET*-bundle, shift operators on $\mathscr{H}$, and semi-infinite forms of $T_{1}^{*} M$. Using Pickrell's quasi-invariant cylinder measure on $\operatorname{Gr}(\mathscr{H})_{0}$ (and the fact that $M \subset \mathrm{GL}_{\text {res }}$ ), Wiesbrock obtains a quasiinvariant measure on $\operatorname{Diff}_{W}$ and an action of $\operatorname{Gr}(\mathscr{H})$ on the space of classical strings.

Enlarging now the string functionals to functionals on the product of string space with $\operatorname{Gr}(\mathscr{H})$, multiplication of two such functionals is defined as convlution on the midpoint, integration over all possible splittings for the given fixed left and right halves (as for $\mathscr{A}$ ) and integration over all possible reparametrisations identified with elements of $\operatorname{Gr}(\mathscr{H})$. Radon-Nikodym derivatives are taken into account. 
Whilst in general this multiplication is not associative, the claim is that the Radon-Nikodym derivatives cancel when $d=26$ to provide an invariant measure and an associative multiplication. (The proof of the latter rests on the analysis of the line bundles over Diff $_{W}$.) This structure is claimed to produce a $\mathrm{C}^{*}$-algebra, appropriate for interacting strings.

Using the Gaussian cylinder measures, Fock-representations are easily obtained with the right creation and annihilation operators, and the Faddeev-Popov ghosts are defined as operators on $\mathscr{H}$ through exterior multiplication of the basis elements of $T_{1} M$ (identified with the $L_{n}$ in our case). However, this cannot reproduce a BRST-charge, because the space is Hilbert, hence no proposed BRSTcharge can be both selfadjoint and 2-nilpotent.

To compare this with our approach, we will not dwell on the somewhat impenetrable technical details, but point out general theoretical differences.

1) Our approach is less ambitious, in that we only aimed at obtaining a $\mathrm{C}^{*}$-algebra which can reproduce the operator theory of the free string - once that was made precise. Time evolution automorphisms and Poincaré transformations were included. Wiesbrock, on the other hand, models the interacting string, omitting time evolution and Poincaré transformations. (Since his strings are in $\mathbb{R}^{d}$, he would need to have sheets in $\mathbb{R}^{d+1}$ rather than paths.) So Wiesbrock models kinematics only, the dynamics is yet to be provided. Even classically one would like to have a time evolution for which a string can be seen to go through its splittings and joinings, with some coupling constant to control the strength of the interaction.

2) The space geometry of the string is explicit in Wiesbrock's approach in that the classical strings as (discontinuous) paths is the starting point for his algebra. In our case the geometry was flushed out at the heuristic level by taking the Fourier transformed theory on the world-sheet (assumed as given). Quantization then proceeded by replacing the oscillator modes with quantum oscillators and retaining the rest of the formalism. In Wiesbrock's case, quantizing was done through defining a new product on string functionals, as described above. There is no way in which we can see how to imbed our theory into Wiesbrock's; multiplication in his case intimately involves the splittings and joinings, and there is no obvious way to switch these off to produce a free string. In fact, whilst Wiesbrock's algebra can produce Fock representations, we cannot see how to identify any Weyl relation in his algebra, so we are not sure why it is said to have a "Bosonic part".

3) In Wiesbrock's approach the reparametrisations fix the points at $0, \frac{1}{2}, 1$, producing the group Diff ${ }_{W}$ as the restricted symmetry group. There is no such restriction in our picture.

4) Wiesbrock's finding that his Gaussian measure is not invariant under reparametrisations, we would like to identify with Bowick and Rajeev's finding that the complex structure of their Fock representation is not invariant under reparametrisation and with our finding that the Fock-Krein complex structure is not invariant under the gauge group. However, we deal with Krein representations for Poincaré covariance, whilst Wiesbrock is concerned with Hilbert representations.

5) Wiesbrock claims his $\mathrm{C}^{*}$-algebra is only defined when $d=26$ (when his multiplication is associative), but in our case there is no such difficulty, and we only expect the dimension 26 restriction to arise in requiring the vacua of particular Fock representations to satisfy the constraints. 
6) It is still necessary for both approaches to examine the existence and structure of the physical states and representations, i.e. those satisfying the constraints.

35. Witten, E.: Non-commutative Geometry and String Field Theory, Nucl. Phys. B268, 253-294 (1986)

Communicated by H. Araki 
